\title{
Orientation Dependence of the Acoustic Backscatter for \\ Elongated Zooplankton
}

by

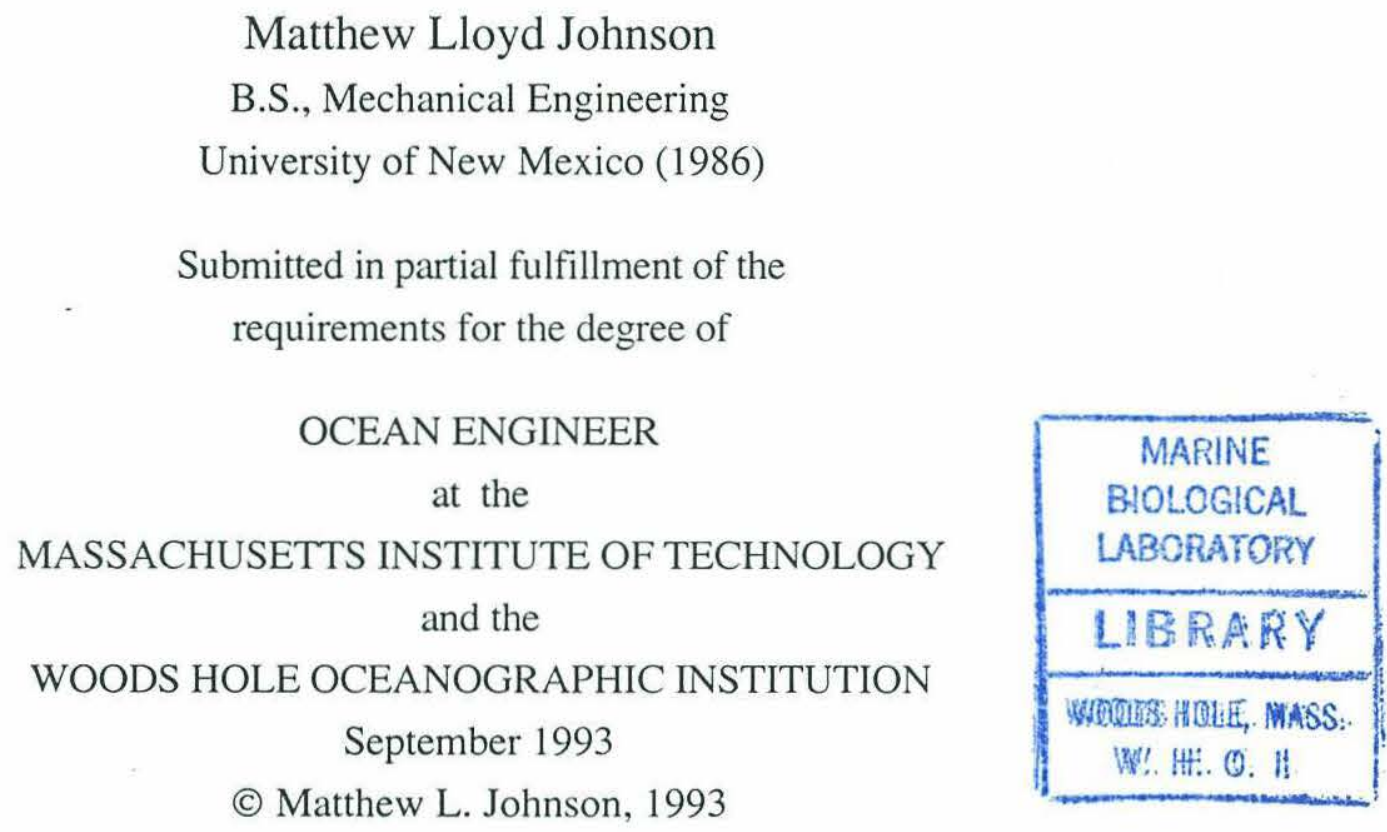

The author hereby grants to MIT, WHOI and the U.S. Government

permission to reproduce and to distribute copies of this thesis document in whole or in part.

Signature of Author?

Joint Program in Applied Ocean Science and Engineering Massachusetts Institute of Technology

Woods Hole Oceanographic Institution

Certified by

Dr. Timothy K. Stanton

Associate Scientist

Woods Hole Oceanographic Institution

Thesis Supervisor

Accepted by

Dr. Arthur B. Baggeroer

Chairman, Joint Committee for Applied Ocean Science and Engineering Massachusetts Institute of Technology / Woods Hole Oceanographic Institution

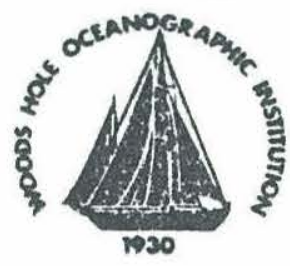




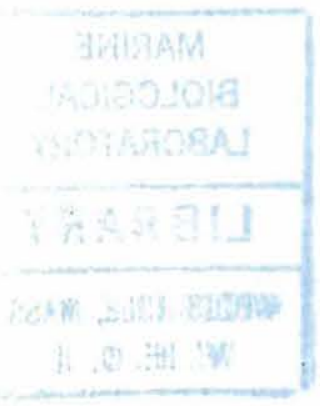




\title{
Orientation Dependence of the Acoustic Backscatter for
}

\section{Elongated Zooplankton}

by

\author{
Matthew Lloyd Johnson
}

\author{
Submitted to the Massachusetts Institute of Technology / \\ Woods Hole Oceanographic Institution \\ Joint Program in Applied Ocean Science and Engineering \\ in partial fulfillment of the \\ requirements for the degree of \\ Ocean Engineer
}

\begin{abstract}
The width of the main lobe of the acoustic backscatter directivity pattern of decapod shrimp (Palaemonetes vulgaris) is examined versus acoustic frequency. Using the distorted wave Born approximation (DWBA) and the geometry of a prolate spheroid, an analytic formula for the backscatter cross section as a function of orientation angle is derived. A directivity pattern is determined from the analytic formula and the width of the main lobe (beamwidth) is computed. The relationship between beamwidth and acoustic frequency is presented in plots of beamwidth versus $k a$ and $L / \lambda$. The model is adapted to experimental limitations of animal motion, discrete sampling and observed side lobe levels. The backscatter directivity patterns of live decapod shrimp, determined experimentally at frequencies between 72 and $525 \mathrm{kHz}$, are presented. A non-monotonic relationship between beamwidth and frequency is illustrated in this study. This relationship is in contrast to the monotonic relationship exhibited when sound scatters off of an impenetrable flat plate. Reasonable agreement is found between the theoretically predicted beamwidths and most experimental data, where the beamwidth was more-or-less oscillatory about a mean value of $19^{\circ}$. The structure can at least be partly explained by scattering theory.
\end{abstract}

Thesis Supervisor: Dr. Timothy K. Stanton

Associate Scientist

Woods Hole Oceanographic Institution 


\section{Acknowledgments}

Sincere thanks are expressed to the U.S. Navy for giving me this opportunity.

I would like to express special thanks, first and foremost, to my advisor, Tim Stanton. Without his patience and guidance throughout the last two years, I would have thrown in the towel long ago. He always provided enthusiastic support and kept me focused on the project when I would stray.

I am deeply indebted to Dezhang Chu. In the laboratory, it was Dr. Chu who taught how to tie a live shrimp with a human hair. Outside the laboratory, he taught me how to integrate a scattering equation for a prolate spheroid. In between he answered hundreds of questions about how to run the experiments.

I am grateful to Dr. Charles Greenlaw of Tracor Applied Science Inc., for permission to use his data on scattering from Euphausia pacifica gathered in 1990. He was also most helpful in responding to questions on his performance of similar experiments with zooplankton.

Special appreciation is due to Steve Bowen, Bob Eastwood, and Doug Ray for their immeasurable patience in teaching me to use UNIX and DOS. I would also like to recognize Paul Boutin and Ann Henry for their constant availability and help in manufacturing or acquiring materials.

Finally, I must thank my wife, Trisha, for her continued support. She knew when to volunteer her help (her proof reading was meticulous and timely), and when to leave the room if things were not going well.

The support, effort and patience of those above made this project fascinating and enjoyable. 


\section{Table of Contents}

List of Figures................................................

List of Tables...................................................9

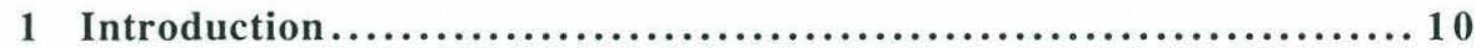

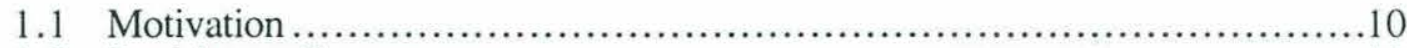

1.2 Problem Statement............................................................1

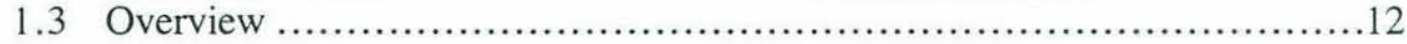

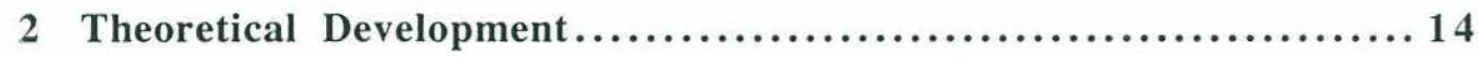

2.1 Helmholtz-Huygens Integral .......................................... 14

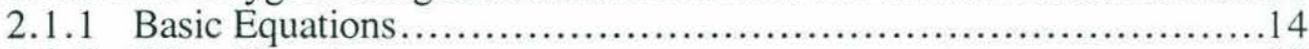

2.1.2 Wave Equation.................................................. 18

2.1.3 Integral Solution to the Wave Equation ...........................21

2.2 Backscatter From a Weak Scatterer...............................................24

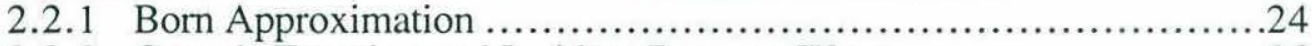

2.2.2 Green's Function and Incident Pressure Wave .....................26

2.3 Prolate Spheroid Geometry....................................................29

2.3.1 Shape of Model and the Definition of Integration Limits ..............29

2.3.2 Solution of the Integral for the Prolate Spheroid Geometry ............30

2.4 Ideal Backscatter Directivity Pattern .........................................35

2.5 Describing Simulated Data to Approximate Experimental Limitations .........43

3 Experimental Measurements ................................ 48

3.1 Overview of Apparatus and Procedures …................................48

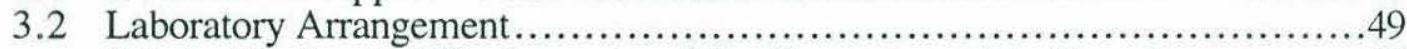

3.2.1 Mechanical Equipment and the Geometry of the Experiment............49

3.2.2 Equipment Set-up and Connections ...............................61

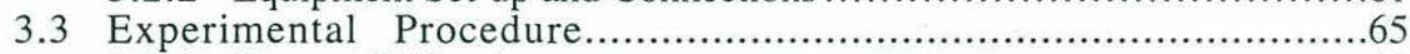

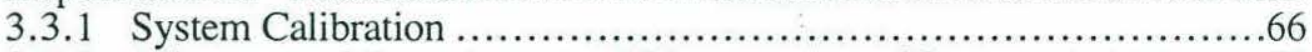

3.3.2 Alignment of Transducers and Measurement of Systematic Noise ....67

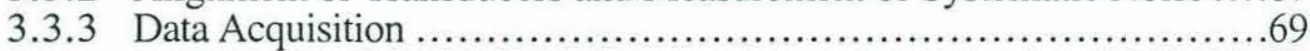

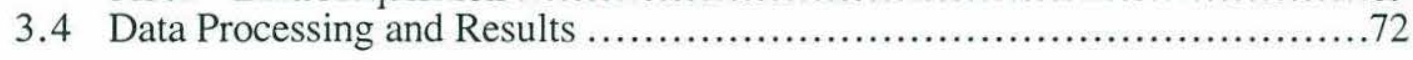

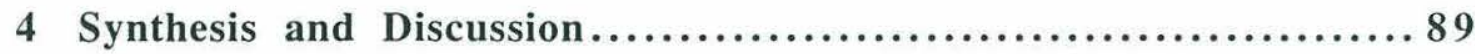

5 Conclusions............................................... 97

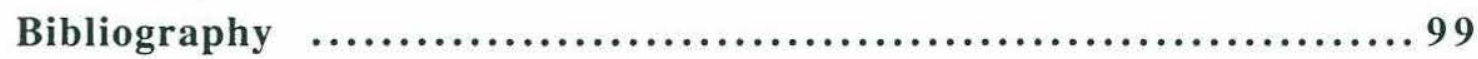




\section{List of Figures}

Figure 1-1

Decapod shrimp (Palaemonetes vulgaris), a common nearshore species of shrimp. (This species is used because it is readily available, and is similar in size and shape to many oceanic species of zooplankton. The animal is shown with the tail curled. The animal normally has its tail extended, so its

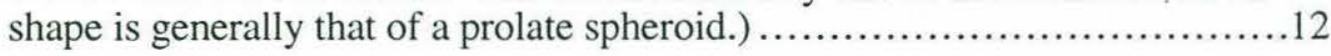

Figure 2-1

Forces acting on an Eulerian differential fluid volume .......................16

Figure 2-2

Particle velocities acting on the faces of a differential fluid volume...............17

Figure 2-3

Discrete inhomogeneity within a homogeneous medium............................19

Figure 2-4

Geometry for the Green's function, the incident pressure wave, and the angle between the discrete volume's major axis and the receiver (the orienta-

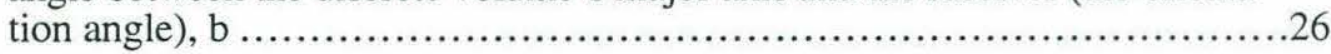

Figure 2-5

Cylindrical coordinates and limits on the prolate spheroid for integration ......29

Figure 2-6

Normalized backscatter directivity patterns for a weak scattering prolate spheroid in a homogeneous medium. The nature of the shape changes in the directivity pattern made it necessary to define the beamwidth of the main lobe (BW) as the width of the pattern at the point where the backscatter cross section $\left(\sigma_{\mathrm{bs}}\right)$ is one-half the maximum value. The beamwidth in each subplot is shown as a solid horizontal line. The acoustic frequency (f) is given in kilohertz. Note the increase in beamwidth between 175 and $200 \mathrm{kHz}$ and between 325 and $350 \mathrm{kHz}$. This increase is due to the method of defining the beamwidth.

Figure 2-7

Comparison of backscatter directivity patterns for penetrable and impenetrable models [(-): backscatter directivity pattern for penetrable volume; (- -): backscatter directivity pattern for impenetrable surface.] .................39

Figure 2-8

Beamwidth of the main lobe of the backscatter directivity pattern for a weak scattering prolate spheroid (-) and an impenetrable flat plate (- $)$. For this

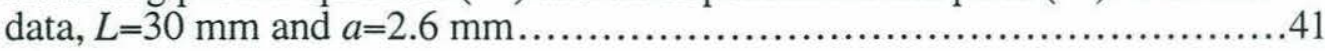


Figure 2-9

Effect of animal sizes on plots of beamwidth versus $k a$ and $L / \lambda$. [(-): $L=34 \mathrm{~mm}, a=2.7 \mathrm{~mm},(L / 2 a)=6.3 ;(-): L=30 \mathrm{~mm}, a=2.6 \mathrm{~mm}$,

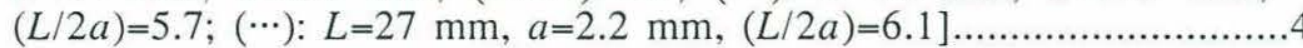

Figure 2-10

Typical angular deviation added to the desired orientation angle. The angular deviation is a zero-mean Gaussian random variable with a $3^{\circ}$ variance averaged over five pings. This factor was used to account for the movement

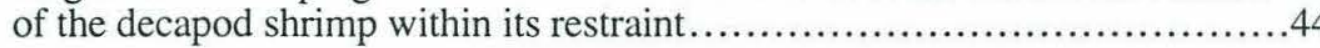

Figure 2-11

Comparison of the backscatter directivity pattern for an "ideal" non-moving prolate spheroid (- -) and the backscatter directivity pattern of the "curve fitted" prolate spheroid with animal motion within the restraint (-). ["Ideal" $\left(\mathrm{BW}_{\mathrm{id}}\right)$ and "curve fitted" $\left(\mathrm{BW}_{\mathrm{cf}}\right)$ beamwidths are shown as solid horizontal lines.]

Figure 2-12

Mean beamwidth of the main lobe of the backscatter directivity pattern for a weak scattering prolate spheroid. (For this data, $L=30 \mathrm{~mm}$ and $a=2.6 \mathrm{~mm}$. Also depicted are the mean beamwidth plus/minus the standard deviation, and the maximum and minimum beamwidths computed.)

Figure 3-1

Target frame used to suspend a live animal in a seawater tank. [The top and bottom are made of stainless steel rods, $4 \mathrm{~mm}$ in diameter. The vertical supports consist of four-pound test fishing line (monofilament). The animal is tethered at the head and tail with a length of human hair.]

Figure 3-2

The target frame - transducer geometry designed to prevent the monofilament supports from interfering with the backscattered pressure signal from the shrimp (target). Top, geometry for end-on target orientation; bottom,

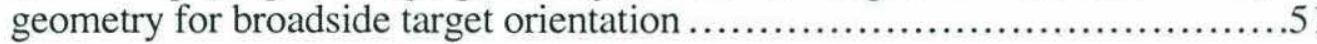

Figure 3-3

Transducer frame used to hold acoustic transducers in position for the experiment. (The transducers were held rigidly in custom made nylon blocks. The nylon blocks could be positioned vertically, and locked in place on $16 \mathrm{~mm}$ stainless steel rods. The rods could be positioned within the frame and rotated to the desired location, and then locked in place. This provided sufficient control of transducer position to perform the experiment.) ..........54

Figure 3-4

Seawater tank in which the experiment was performed. (The 11,000 liter

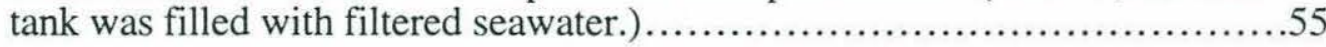

Figure 3-5

Geometry for determining the range to the farfield of a piston transducer. .....57

Figure 3-6

Geometry for determining the diameter of the first Fresnel zone $\left(D_{f z}\right)$, at range $r$, from the transducer

Figure 3-7

Schematic diagram of data acquisition system 
Figure 3-8

Transducer configuration for calibration of the data acquisition system. ( $R$ is the range between transducer faces. During the actual measurements, the range was $98 \mathrm{~cm}$, vice $100 \mathrm{~cm}$, due to physical limitations within the transducer frame.)

Figure 3-9

Configuration for transducer alignment. ( $r$ is the range from the transducer face to the target; $s$ is the centerline separation between the transducers; a is defined as the "transducer angle".)

Figure 3-10

Typical backscattered pressure wave from a decapod shrimp. (This was collected at broadside orientation of an animal insonified at an acoustic frequency of $167 \mathrm{kHz}$. The horizontal scale is the time, in milliseconds, since the incident pressure wave was initiated. The incident pressure wave was a sinusoid with an amplitude of 2 volts, and a pulse length of $0.1 \mathrm{msec}$.)........72

Figure 3-11

Examples of measured backscatter directivity patterns for decapod shrimp ...75-81

Figure 3-12

Directivity patterns from "chirp" data. This is the starboard aspect of the animal, with the head at $180^{\circ}$ and the telson at $0^{\circ}$

Figure 4-1

Experimentally and theoretically determined beamwidths plotted against the characteristic parameters, $k a$ and $L / \lambda$. ('o' denotes narrowband data; '*' and ' $\oplus$ ' denote chirp data, the '*' data involves including a "rogue" side lobe in the estimate of beamwidth; and ' + ' denotes Greenlaw's data. The theoretical data has modeled animal motion and sampling limitations.) ........91

Figure 4-2

Theoretical backscatter target strength near end-on incidences. (The normalized target strength is the maximum target strength for orientation angles between $0^{\circ}$ and $10^{\circ}$. It is normalized to the maximum target strength of the

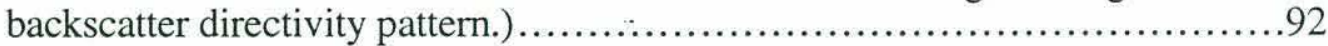

Figure 4-3

Comparison of a theoretical backscatter directivity pattern with a "pedestal" (-), and an experimentally determined backscatter directivity pattern (- -) ...93

Figure 4-4

Experimentally measured beamwidths and theoretical data with a "pedestal" plotted against $k a$. and $L / \lambda$.. ('o' denotes narrowband data; ' $*$ ' and ' $\oplus$ ' denote chirp data, the ' $*$ ' data involves including a "rogue" side lobe in the estimate of beamwidth; and '+' denotes Greenlaw's data. The theoretical data has modeled animal motion, sampling limitations, and appendage motion.)

Figure 4-5

Comparison of the normalized mean cross section of the "ideal" nonmoving prolate spheroid (-), an impenetrable plate (- -), and experimental data ('o' denotes narrowband data and ' + ' denotes chirp data) ...............96 


\section{List of Tables}

Table 3-1

Sound Speeds $(\mathrm{m} / \mathrm{s})$ for Various Temperatures and Salinities....................55

Table 3-2

Distance to Farfield $\left(R_{f f}\right)$ for Various Transducers $(c=1520 \mathrm{~m} / \mathrm{s}) \ldots \ldots \ldots \ldots \ldots . . .58$

Table 3-3

Diameters of the First Fresnel Zone for Ranges of 50 and $100 \mathrm{~cm} \ldots \ldots \ldots \ldots 61$

Table 3-4

Electronic Equipment used in the Data Acquisition System......................63

Table 3-5

Bandpass Filter Settings ..............................................63

Table 3-6

Transducer Angles at Ranges of 54 and 107 centimeters ...................68

Table 3-7

Animal Size and Data Sampled in the Experiments .......................71

Table 3-8

Experimentally Measured Beamwidths from Narrowband Analyses..........82

Table 3-9

Experimentally Measured Beamwidths from Broadband Analyses............84

Table 3-10

Data from Greenlaw's Measurements of Euphausia pacifica (1990) ..........88 


\section{Chapter 1}

\section{Introduction}

\subsection{Motivation}

Plankton play a primary role in the food chain of the ocean [1]. Measurement of the abundance, diversity, and size distribution of plankton is vital to understanding the resources present in the ocean. Accurate, quantitative measurements of these resources will allow for the trends in these resources to be determined. By measuring these trends in organisms at the base of the food chain, the effects of environmental changes (whether natural or man-made) can be more accurately and rapidly predicted.

Present methods of measuring the abundance and diversity of zooplankton in the ocean rely upon sampling with various types of plankton nets. Net sampling leads to inaccuracies by only being able to sample a very small region, and then extrapolating the results to a much larger region. Also, there is a significant time lag between sampling and determining the results. Finally, the plankton net is invasive, and as many zooplankton are freeswimmers, the animals avoiding the net will cause further inaccuracies in the data gathered.

Advanced methods of acoustically measuring populations of larger zooplankton, such as decapod shrimp (Palaemonetes vulgaris), are currently being developed $[2,3,4]$. Acoustic measurements of zooplankton allow greater areas of the ocean to be investigated at a faster rate than with plankton nets. Additionally, acoustic measurements are noninvasive and much more suitable to the study of free-swimming zooplankton [5]. 
To remotely estimate zooplankton populations, a scattering model for the species of interest must be known $[5,6]$. The target strength predicted from these models is strongly dependent upon the size, shape, acoustic frequency, material properties (mass density and speed of sound contrasts) and orientation. By measuring the target strength of the zooplankton being investigated over a range of frequencies, the size distribution and abundance of the organisms can be determined $[7,8,9]$. (For a given species, the shape and material properties can be accounted for in the development of the scattering model.)

\subsection{Problem Statement}

The development of a useful acoustic backscattering model for elongated zooplankton, specifically decapod shrimp (Palaemonetes vulgaris), is a very complex task. The simplest model is the fluid sphere [10]. Greenlaw used this model for zooplankton, but discovered that the scattering behavior is influenced by shape [11]. As seen in Fig. 1-1, elongated animals cannot be modeled sufficiently by a simple mathematical shape, such as a sphere [2].

In a series of papers, Stanton developed an approximate model for the acoustic backscattering from deformed elongated bodies $[12,13,14]$. Stanton and colleagues later simplified the approach by using a ray theory so that simple, yet accurate descriptions of the scattering by fluid-like animals were possible $[3,4]$. The good comparison between the new models and data suggests that certain elongated zooplankton behave acoustically as finite cylinders.

This thesis complements the work of Chu et al [2] by measuring the orientation dependence of individual animals over a wide range of acoustic frequencies. Similar work has been done by Greenlaw, but over a more limited frequency range [15]. The back- 
scattered pressure from these animals is highly orientation dependent. The main lobe of the backscatter directivity pattern corresponds to angles of incidence near broadside. This thesis will present a relationship between the width of the main lobe and acoustic frequency for Palaemonetes vulgaris.

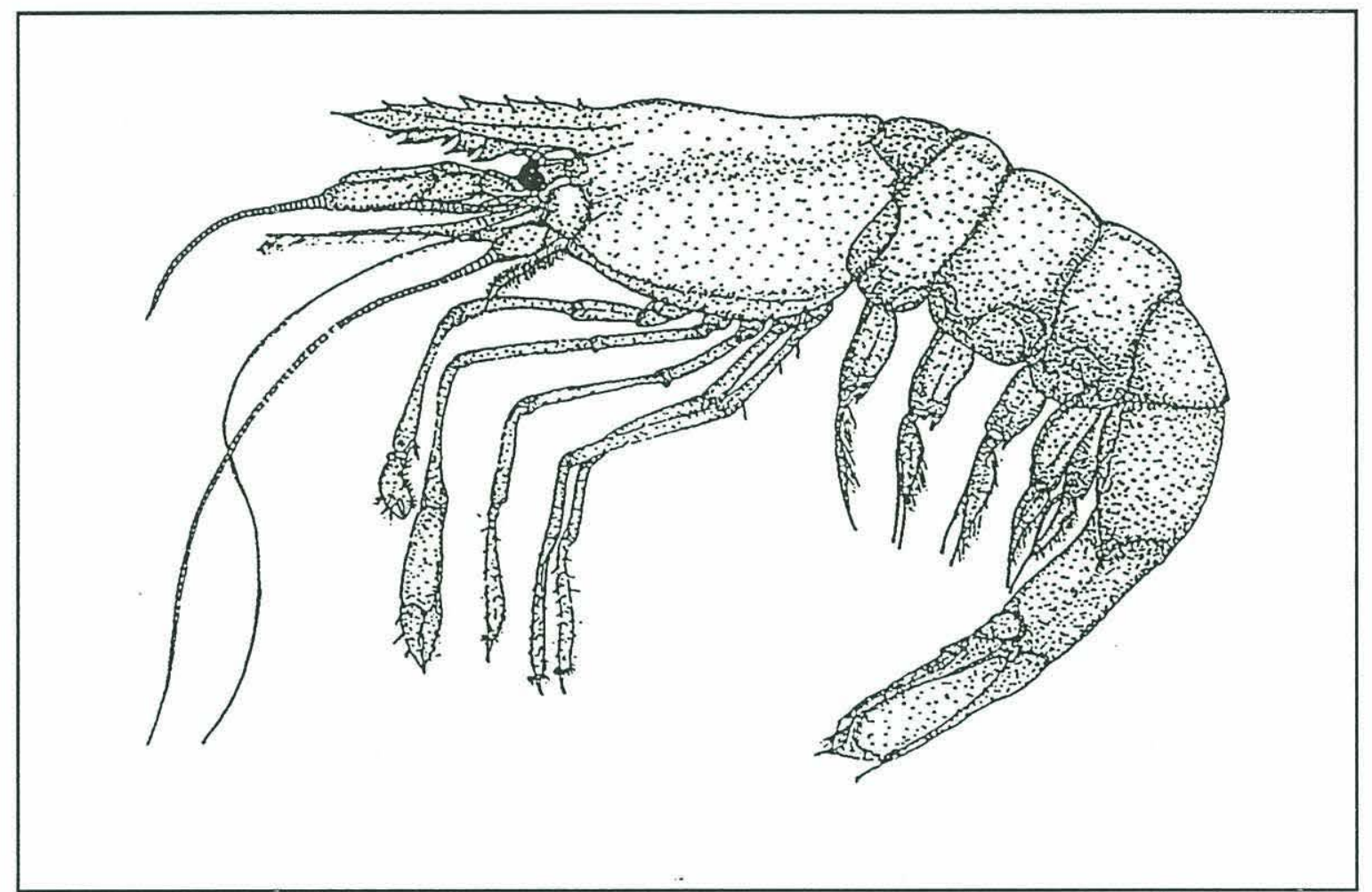

Figure 1-1: Decapod shrimp (Palaemonetes vulgaris), a common nearshore species of shrimp. (This species is used because it is readily available, and is similar in size and shape to many oceanic species of zooplankton. The animal is shown with the tail curled. The animal normally has its tail extended, so its shape is generally that of a prolate spheroid.)

\subsection{Overview}

Chapter 2 gives the development of the model used to predict the width of the main lobe of the backscatter directivity pattern as a function of acoustic frequency. The deformed cylinder model used by Stanton et al [3] would be inappropriate for this analysis, as 
their model is designed for averages over angle of orientation rather than instantaneous realizations at any given angle. In their approach, the model is more accurate near broadside incidence. The distorted wave Born approximation (DWBA) lends itself more readily for predicting the target strength as a function of orientation angle for all orientation angles. The geometry of a prolate spheroid is used as it approximates the geometric shape of the animal [11]. The formulation of the Helmholtz-Huygens integral is presented and simplified using the DWBA. An expression for the backscatter directivity pattern is developed by applying the geometry of the prolate spheroid to the DWBA integral, and solving the integral. This analytic model is then adjusted to reflect practical limitations encountered in the experiment. Specifically, the model is corrected for the motion of the live shrimp and sampling limitations within the experimental data.

Chapter 3 details the experimental procedure, in which individual live decapod shrimp were tethered and rotated through a full circle while being insonified with frequencies from 72 to $525 \mathrm{kHz}$. The backscattered pressure wave was recorded as a function of frequency and orientation angle of the animal. From this data, the actual backscatter directivity pattern for the animals was produced.

Chapters 4 and 5 discuss and summarize the results, and present conclusions. 


\section{Chapter 2}

\section{Theoretical Development}

\subsection{Helmholtz-Huygens Integral}

\subsubsection{Basic Equations}

The Helmholtz-Huygens integral is a solution to the three dimensional wave equation. The three dimensional wave equation is derived from the linearized forms of three basic equations: the adiabatic equation of state, the Euler equation, and the continuity equation [16].

These three equations relate the variables pressure, $p$, density, $\rho$, and particle velocity, $\mathbf{v}$. The total pressure consists of two components, a hydrostatic pressure, $p_{0}$, and a dynamic or "excess" (sound) pressure $p_{1}$ :

$$
p=p_{0}+p_{1} .
$$

The hydrostatic pressure is assumed constant, so the differential pressure, $d p$, is equal to:

$$
d p=d p_{1} .
$$

Similarly for particle velocity, there is a static and a dynamic (sound) component:

$$
\begin{aligned}
& \mathbf{v}=\mathbf{v}_{0}+\mathbf{v}_{1} \\
& d \mathbf{v}=d \mathbf{v}_{1} .
\end{aligned}
$$


For an inhomogeneous media, there are three components to density. There is a hydrostatic density, $\rho_{0}$, there is the local change in static density, $\Delta \rho_{0}$, and there is the dynamic (sound) density, $\rho_{1}$ :

$$
\begin{gathered}
\rho=\rho_{0}+\Delta \rho_{0}+\rho_{1} \\
d \rho=d \rho_{1} .
\end{gathered}
$$

Both the hydrostatic density and local change in static density are assumed to be much greater than the dynamic density.

The adiabatic state equation asserts that the pressure is a function of density:

$$
p=f(\rho) .
$$

The differential pressure can then be represented as a Taylor series in terms of $f(\rho)$ :

$$
d p=\left(\frac{\partial f(\rho)}{\partial \rho}\right)_{p=p_{0}} d \rho+\left(\frac{\partial^{2} f(\rho)}{\partial \rho^{2}}\right)_{p=p_{0}} \frac{(d \rho)^{2}}{2}+\cdots
$$

Since the changes in pressure and density are assumed to be small, the higher order terms can be neglected. Also, this lets us define

$$
c^{2} \equiv\left(\frac{\partial f(\rho)}{\partial \rho}\right)_{p=p_{0}}
$$

and assume, $c^{2}=$ constant. Thus, Eq. (2.8) simplifies to :

$$
d p=c^{2} d \rho .
$$

Integrating Eq. (2.10) produces the linearized adiabatic state equation:

$$
p=c^{2} \rho+\text { constant } .
$$

To clear the constant term, we take the total derivative of Eq. (2.11):

$$
\frac{d p}{d t}=c^{2} \frac{d \rho}{d t} .
$$

Since pressure varies slowly with position over regions small compared to the wavelength, the adiabatic equation of state can be written as

$$
\frac{\partial p}{\partial t}=c^{2}\left[\frac{\partial \rho}{\partial t}+\nabla \rho \bullet \mathbf{v}\right]
$$

Euler's equation is derived from the linearized conservation of momentum for a fluid bundle. The name "Euler" comes from the use of the Euler description, in which the 
coordinate system is fixed in space and the fluid is observed to move past the fixed reference point. The Euler equation is found by applying Newton's Second Law of Motion $(\mathbf{F}=m d \mathbf{v} / d t)$ to the Eulerian fluid bundle shown in Fig. 2-1. The force balance (the left hand side of Newton's Second Law) on the fluid bundle is given as:

$$
\mathbf{F}=[p(x)-p(x+d x)] d y d z \mathbf{e}_{x}+[p(y)-p(y+d y)] d x d z \mathbf{e}_{y}+[p(z)-p(z+d z)] d x d y \mathbf{e}_{z} .
$$

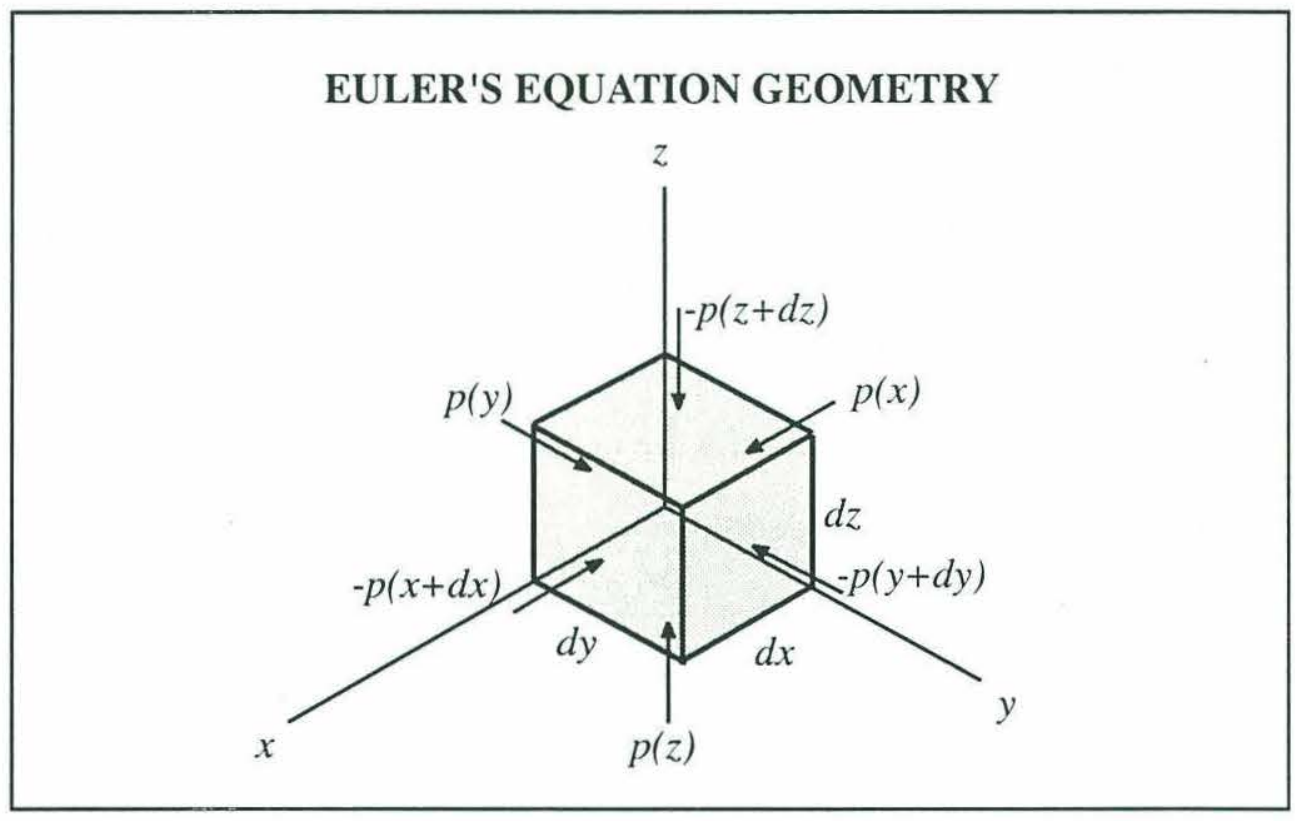

Figure 2-1: Forces acting on an Eulerian differential fluid volume.

The right hand side of Newton's Second Law is given as:

$$
m d \mathbf{v} / d t=\rho d V\left(\frac{\partial \mathbf{v}}{\partial t}+\nabla \mathbf{v} \bullet \mathbf{v}\right) .
$$

Dividing Eqs. (2.14) and (2.15) by $d V=d x d y d z$, reducing the left hand side and setting the results equal to each other yields:

$$
-\nabla p=\rho\left(\frac{\partial \mathbf{v}}{\partial t}+\nabla \mathbf{v} \bullet \mathbf{v}\right) .
$$

The Euler equation can be further reduced by assuming that $\mathbf{v}$ is small (thus the second order term $\nabla \mathbf{v} \bullet \mathbf{v}$ becomes negligible). The resulting equation is:

$$
-\nabla p=\rho \frac{\partial \mathrm{v}}{\partial t}
$$


The continuity equation provides the third relationship between particle velocity, pressure and density. The equation is derived by applying the principle of conservation of mass to a small volume (Fig. 2-2). The principle of conservation of mass states that the difference between the mass entering the volume and the mass leaving the volume is the change in mass in the volume:

$$
m_{\text {in }}-m_{\text {out }}=\Delta m
$$

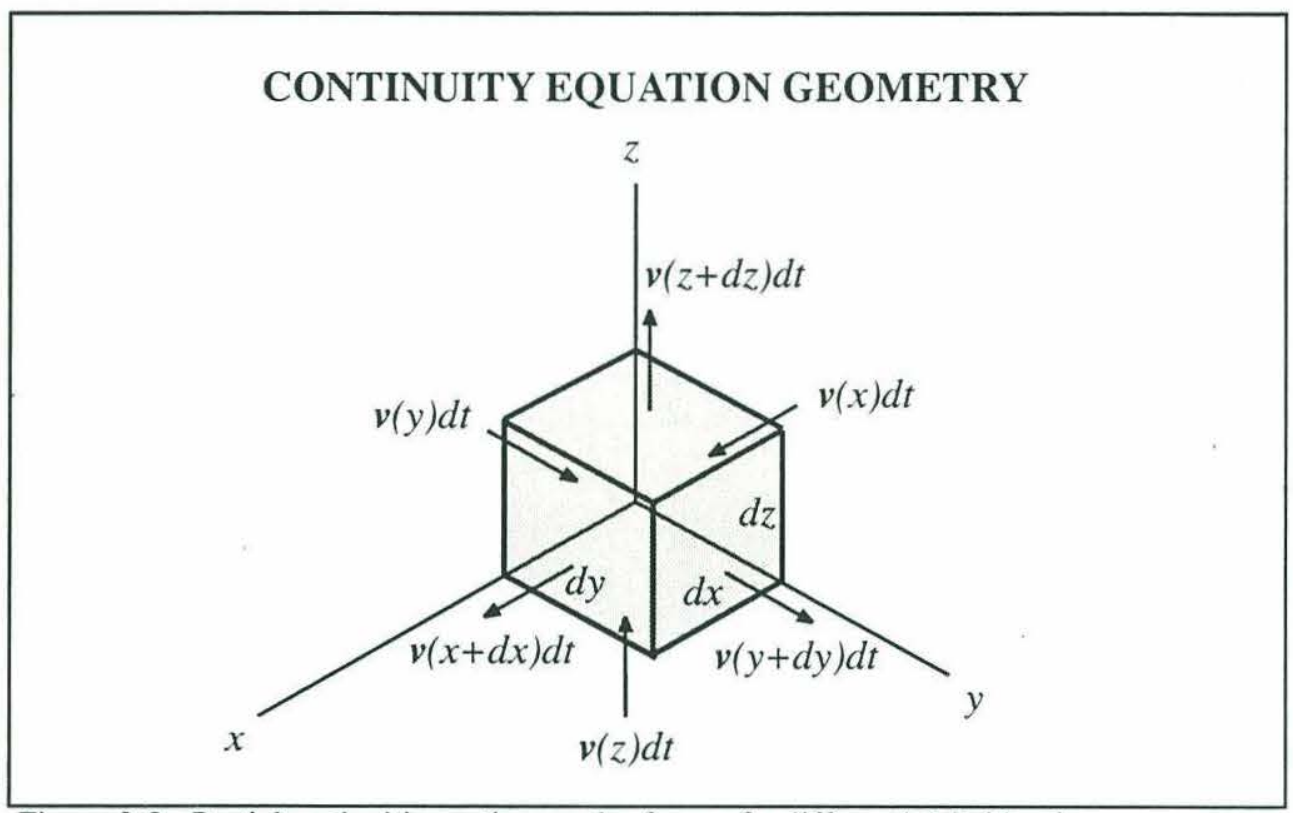

Figure 2-2: Particle velocities acting on the faces of a differential fluid volume.

The mass entering the differential volume is:

$$
m_{i n}=[\rho(x) \mathbf{v}(x) d y d z+\rho(y) \mathbf{v}(y) d x d z+\rho(z) \mathbf{v}(z) d x d y] d t .
$$

The mass leaving the differential volume is:

$$
m_{\text {out }}=[\rho(x+d x) \mathbf{v}(x+d x) d y d z+\rho(y+d y) \mathbf{v}(y+d y) d x d z+\rho(z+d z) \mathbf{v}(z+d z) d x d y] d t .
$$

Subtracting Eq. (2.20) from Eq. (2.19), and converting to vector notation:

$$
m_{\text {in }}-m_{\text {out }}=-\nabla \cdot(\rho \mathbf{v}) d t d x d y d z .
$$

The mass change within the differential volume is:

$$
\Delta m=\frac{\partial \rho}{\partial t} d t d x d y d z
$$


Equating Eqs. (2.21) and (2.22), then canceling like terms, produces:

$$
\begin{gathered}
-\nabla \bullet(\rho \mathbf{v})=\frac{\partial \rho}{\partial t} \\
-(\nabla \rho \bullet \mathbf{v}+\rho \nabla \bullet \mathbf{v})=\frac{d \rho}{d t}-\nabla \rho \bullet \mathbf{v} \\
-\nabla \bullet \mathbf{v}=\frac{1}{\rho} \frac{d \rho}{d t} .
\end{gathered}
$$

Applying Eq. (2.12) to the right hand side of Eq. (2.23) yields:

$$
-\nabla \cdot \mathbf{v}=\frac{1}{\rho c^{2}} \frac{\partial p}{\partial t} \text {. }
$$

In summary, we have:

$$
\begin{aligned}
\frac{\partial p}{\partial t} & =c^{2}\left(\frac{\partial \rho}{\partial t}+\nabla \rho \bullet \mathbf{v}\right) & & (\text { equation of state) } \\
\frac{d \mathbf{v}}{d t} & =-\frac{1}{\rho} \nabla p & & \text { (Euler's equation) } \\
\nabla \bullet \mathbf{v} & =-\frac{1}{\rho c^{2}} \frac{\partial p}{\partial t} & & \text { (continuity equation). }
\end{aligned}
$$

\subsubsection{Wave Equation}

The previous section developed a set a three equations relating three variables (particle velocity, density and pressure). These three equations can be manipulated into one equation and one unknown - this is the three dimensional wave equation.

The first step is to relate Euler's equation to the continuity equation, and eliminate particle velocity as a variable. This is done by taking the divergence of Euler's equation [Eq. (2.26)]

$$
\nabla \bullet \frac{d \mathbf{v}}{d t}=-\nabla \bullet\left(\frac{1}{\rho} \nabla p\right),
$$

and the time derivative of the continuity equation [Eq. (2.27)]

$$
\frac{d}{d t}(\nabla \bullet \mathbf{v})=-\frac{d}{d t}\left(\frac{1}{\rho c^{2}} \frac{d p}{d t}\right)
$$


Then setting them equal to each other, recalling that

$$
\nabla \cdot \frac{d \mathbf{v}}{d t}=\frac{d}{d t}(\nabla \cdot \mathbf{v})
$$

the result is the wave equation for an inhomogeneous medium:

$$
\nabla \cdot\left(\frac{1}{\rho} \nabla p\right)=\frac{d}{d t}\left(\frac{1}{\rho c^{2}} \frac{d p}{d t}\right) \text {. }
$$

This problem deals with a discrete inhomogeneity within a homogeneous medium (Fig. 2-3). The effect of small inhomogeneities, [such as an animal with constant density, $\rho_{a}$, and sound speed, $c_{a}$, in a homogeneous environment $\left.\left(\rho_{0}, c_{0}\right)\right]$, can be accounted for by adding and subtracting the corresponding terms for the homogeneous medium to the inhomogeneous wave equation [Eq. (2.31)] [16]:

$$
\nabla \cdot\left[\left(\frac{1}{\rho}-\frac{1}{\rho_{0}}\right) \nabla p+\frac{1}{\rho_{0}} \nabla p\right]=\frac{d}{d t}\left[\left(\frac{1}{\rho c^{2}}-\frac{1}{\rho_{0} c_{0}^{2}}\right) \frac{d p}{d t}+\frac{1}{\rho_{0} c_{0}^{2}} \frac{d p}{d t}\right] .
$$

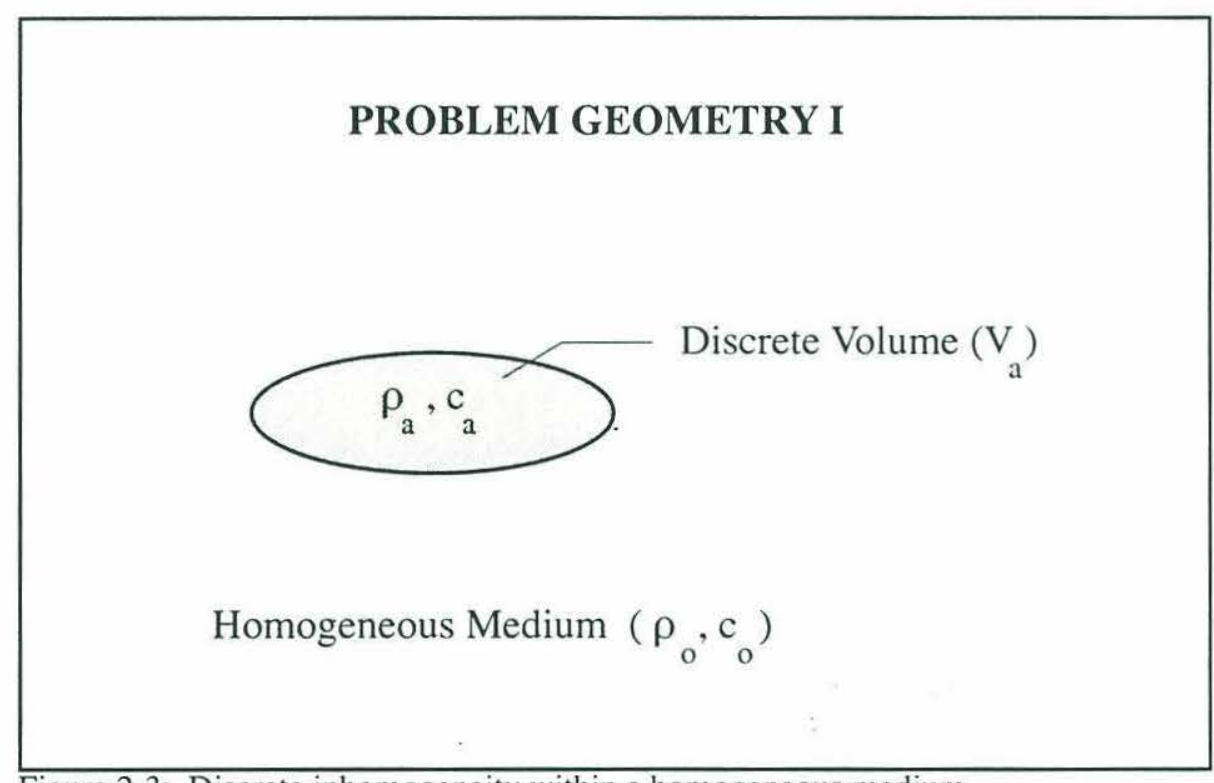

Figure 2-3: Discrete inhomogeneity within a homogeneous medium.

By treating the densities as discrete constants, Eq. (2.32) reduces to:

$$
\begin{gathered}
\nabla \bullet\left[\left(\frac{1}{\rho}-\frac{1}{\rho_{0}}\right) \nabla p+\frac{1}{\rho_{0}} \nabla p\right]=\left(\frac{1}{\rho c^{2}}-\frac{1}{\rho_{0} c_{0}^{2}}\right) \frac{d^{2} p}{d t^{2}}+\frac{1}{\rho_{0} c_{0}^{2}} \frac{d^{2} p}{d t^{2}} \\
\nabla \bullet\left(\frac{\rho_{0}-\rho}{\rho_{0} \rho} \nabla p\right)+\frac{1}{\rho_{0}} \nabla^{2} p=\frac{\rho_{0} c_{0}^{2}-\rho c^{2}}{\rho c^{2} \rho_{0} c_{0}^{2}} \frac{d^{2} p}{d t^{2}}+\frac{1}{\rho_{0} c_{0}^{2}} \frac{d^{2} p}{d t^{2}}
\end{gathered}
$$




$$
\begin{aligned}
& \nabla \cdot\left(\frac{\rho_{0}-\rho}{\rho} \nabla p\right)+\nabla^{2} p=\frac{\rho_{0} c_{0}^{2}-\rho c^{2}}{\rho c^{2}} \frac{1}{c_{0}^{2}} \frac{d^{2} p}{d t^{2}}+\frac{1}{c_{0}^{2}} \frac{d^{2} p}{d t^{2}} \\
& \nabla^{2} p-\frac{1}{c_{0}^{2}} \frac{d^{2} p}{d t^{2}}=\nabla \cdot\left(\frac{\rho-\rho_{0}}{\rho} \nabla p\right)+\frac{\rho_{0} c_{0}^{2}-\rho c^{2}}{\rho c^{2}} \frac{1}{c_{0}^{2}} \frac{d^{2} p}{d t^{2}} .
\end{aligned}
$$

The compressibility $(\kappa)$ of a fluid is:

$$
\kappa=\frac{1}{\rho c^{2}} .
$$

Substituting into Eq. (2.33):

$$
\nabla^{2} p-\frac{1}{c_{0}^{2}} \frac{d^{2} p}{d t^{2}}=\nabla \cdot\left(\frac{\rho-\rho_{0}}{\rho} \nabla p\right)+\frac{\kappa-\kappa_{0}}{\kappa_{0}} \frac{1}{c_{0}^{2}} \frac{d^{2} p}{d t^{2}} .
$$

To further simplify the inhomogeneous wave equation, let us define a density factor $\left(\gamma_{\rho}\right)$ and a compressibility factor $\left(\gamma_{\kappa}\right)$ as:

$$
\begin{gathered}
\gamma_{\rho}(\mathbf{r})=\left\{\begin{array}{cc}
\frac{\rho-\rho_{0}}{\rho} & \mathbf{r} \subset V_{a} \\
0 & \mathbf{r} \not \subset V_{a}
\end{array}\right. \\
\gamma_{\kappa}(\mathbf{r})=\left\{\begin{array}{cc}
\frac{\kappa-\kappa_{0}}{\kappa_{0}} & \mathbf{r} \subset V_{a} \\
0 & \mathbf{r} \not \subset V_{a} .
\end{array}\right.
\end{gathered}
$$

These factors reduce Eq. (2.35) to:

$$
\nabla^{2} p-\frac{1}{c_{0}^{2}} \frac{d^{2} p}{d t^{2}}=\nabla \bullet\left(\gamma_{\rho} \nabla p\right)+\gamma_{\kappa} \frac{1}{c_{0}^{2}} \frac{d^{2} p}{d t^{2}} .
$$

The temporal derivatives can be eliminated by assuming that the pressure is a periodic "pure tone" function [16]:

$$
\begin{aligned}
p & =\tilde{p} e^{i \omega t} \\
\frac{d^{2} p}{d t^{2}} & =-\omega^{2} \tilde{p} e^{i \omega t} \\
& =-\omega^{2} p \\
& =-\left(k_{0} c_{0}\right)^{2} p .
\end{aligned}
$$

Thus, the final form of the inhomogeneous wave equation is [17]:

$$
\nabla^{2} p+k_{0}^{2} p=\nabla \bullet\left(\gamma_{\rho} \nabla p\right)-\gamma_{\kappa} k_{0}^{2} p .
$$




\subsubsection{Integral Solution to the Wave Equation}

The inhomogeneous wave equation is solved by use of the Green's function. The Green's function, $g\left(\mathbf{r} \mid \mathbf{r}_{0}\right)$, is a solution for the unbounded medium, of the equation:

$$
\nabla^{2} g\left(\mathbf{r} \mid \mathbf{r}_{0}\right)+k_{0}^{2} g\left(\mathbf{r} \mid \mathbf{r}_{0}\right)=-\delta\left(\mathbf{r}-\mathbf{r}_{0}\right) .
$$

Here, $g\left(\mathbf{r} \mid \mathbf{r}_{0}\right)$ is the Green's function at observation point $\mathbf{r}$, observing the point $\mathbf{r}_{0}$. The position vectors are:

$$
\begin{aligned}
& \mathbf{r}=x \mathbf{e}_{x}+y \mathbf{e}_{y}+z \mathbf{e}_{z} \\
& \mathbf{r}_{0}=x \mathbf{e}_{x_{0}}+y \mathbf{e}_{y_{0}}+z \mathbf{e}_{z_{0}} .
\end{aligned}
$$

The inhomogeneous wave equation is:

$$
\nabla^{2} p(\mathbf{r})+k_{0}^{2} p(\mathbf{r})=\nabla \bullet\left[\gamma_{\rho}(\mathbf{r}) \nabla p(\mathbf{r})\right]-\gamma_{\kappa}(\mathbf{r}) k_{0}^{2} p(\mathbf{r}) .
$$

By multiplying Eq. (2.41) by $p(\mathbf{r})$ and Eq. (2.43) by $g\left(\mathbf{r} \mid \mathbf{r}_{0}\right)$ the following results are attained:

$$
\begin{gathered}
p(\mathbf{r}) \nabla^{2} g\left(\mathbf{r} \mid \mathbf{r}_{0}\right)+p(\mathbf{r}) k^{2} g\left(\mathbf{r} \mid \mathbf{r}_{0}\right)=-p(\mathbf{r}) \delta\left(\mathbf{r}-\mathbf{r}_{0}\right) \\
g\left(\mathbf{r} \mid \mathbf{r}_{0}\right) \nabla^{2} p(\mathbf{r})+g\left(\mathbf{r} \mid \mathbf{r}_{0}\right) k_{0}^{2} p(\mathbf{r})=-g\left(\mathbf{r} \mid \mathbf{r}_{0}\right) f(\mathbf{r}),
\end{gathered}
$$

where, $f(\mathbf{r})$ is defined as:

$$
f(\mathbf{r}) \equiv-\left\{\nabla \bullet\left[\gamma_{\rho}(\mathbf{r}) \nabla p(\mathbf{r})\right]-\gamma_{\kappa}(\mathbf{r}) k_{0}^{2} p(\mathbf{r})\right\} .
$$

Subtracting Eq. (2.44) from Eq.(2.45):

$$
g\left(\mathbf{r} \mid \mathbf{r}_{0}\right) \nabla^{2} p(\mathbf{r})-p(\mathbf{r}) \nabla^{2} g\left(\mathbf{r} \mid \mathbf{r}_{0}\right)=p(\mathbf{r}) \delta\left(\mathbf{r}-\mathbf{r}_{0}\right)-g\left(\mathbf{r} \mid \mathbf{r}_{0}\right) f(\mathbf{r}) .
$$

Using the reciprocity theorem for Green's functions, the symmetry of the delta function, and interchanging the position vectors $\mathbf{r}$ and $\mathbf{r}_{0}$, Eq. (2.47) can be written as:

$$
g\left(\mathbf{r} \mid \mathbf{r}_{0}\right) \nabla_{0}^{2} p\left(\mathbf{r}_{0}\right)-p\left(\mathbf{r}_{0}\right) \nabla_{0}^{2} g\left(\mathbf{r} \mid \mathbf{r}_{0}\right)=p\left(\mathbf{r}_{0}\right) \delta\left(\mathbf{r}-\mathbf{r}_{0}\right)-g\left(\mathbf{r} \mid \mathbf{r}_{0}\right) f\left(\mathbf{r}_{0}\right),
$$

where,

$$
\nabla_{0} \equiv \frac{\partial}{\partial x_{0}} \mathbf{e}_{x_{0}}+\frac{\partial}{\partial y_{0}} \mathbf{e}_{y_{0}}+\frac{\partial}{\partial z_{0}} \mathbf{e}_{z_{0}}
$$


Integrating over the spatial volume $\left(d V_{0}=d x_{0} d y_{0} d z_{0}\right)$,

$$
\begin{aligned}
& \iiint\left[g\left(\mathbf{r} \mid \mathbf{r}_{0}\right) \nabla_{0}^{2} p\left(\mathbf{r}_{0}\right)-p\left(\mathbf{r}_{0}\right) \nabla_{0}^{2} g\left(\mathbf{r} \mid \mathbf{r}_{0}\right)\right] d V_{0} \\
& =\iiint p\left(\mathbf{r}_{0}\right) \delta\left(\mathbf{r}-\mathbf{r}_{0}\right) d V_{0}-\iiint g\left(\mathbf{r} \mid \mathbf{r}_{0}\right) f\left(\mathbf{r}_{0}\right) d V_{0} .
\end{aligned}
$$

Since the first term on the right hand side is an integral over a delta function,

$$
p(\mathbf{r})=\iiint p\left(\mathbf{r}_{0}\right) \delta\left(\mathbf{r}-\mathbf{r}_{0}\right) d V_{0} .
$$

Thus, Eq. (2.50) can be rewritten as:

$$
p(\mathbf{r})=\iiint\left[g\left(\mathbf{r} \mid \mathbf{r}_{0}\right) \nabla_{0}^{2} p\left(\mathbf{r}_{0}\right)-p\left(\mathbf{r}_{0}\right) \nabla_{0}^{2} g\left(\mathbf{r} \mid \mathbf{r}_{0}\right)\right] d V_{0}+\iiint g\left(\mathbf{r} \mid \mathbf{r}_{0}\right) f\left(\mathbf{r}_{0}\right) d V_{0} .
$$

Noting that the first integrand is the divergence of a vector,

$$
g\left(\mathbf{r} \mid \mathbf{r}_{0}\right) \nabla_{0}^{2} p\left(\mathbf{r}_{0}\right)-p\left(\mathbf{r}_{0}\right) \nabla_{0}^{2} g\left(\mathbf{r} \mid \mathbf{r}_{0}\right)=\nabla_{0} \bullet\left[g\left(\mathbf{r} \mid \mathbf{r}_{0}\right) \nabla_{0} p\left(\mathbf{r}_{0}\right)-p\left(\mathbf{r}_{0}\right) \nabla_{0} g\left(\mathbf{r} \mid \mathbf{r}_{0}\right)\right],
$$

this allows the first integral to be reduced, via the second Green formula, from a volume integral to a surface integral [18],

$$
\begin{aligned}
& \iiint\left[g\left(\mathbf{r} \mid \mathbf{r}_{0}\right) \nabla_{0}^{2} p\left(\mathbf{r}_{0}\right)-p\left(\mathbf{r}_{0}\right) \nabla_{0}^{2} g\left(\mathbf{r} \mid \mathbf{r}_{0}\right)\right] d V_{0} \\
& =\iint\left[g\left(\mathbf{r} \mid \mathbf{r}_{0}\right) \frac{\partial}{\partial n_{0}} p\left(\mathbf{r}_{0}\right)-p\left(\mathbf{r}_{0}\right) \frac{\partial}{\partial n_{0}} g\left(\mathbf{r} \mid \mathbf{r}_{0}\right)\right] d S_{0} .
\end{aligned}
$$

Here, the normal component of the gradient of a function in the outward direction, is defined as $\partial / \partial n_{0}$. The pressure at any position, $\mathbf{r}$, is now described by the equation [16]:

$$
p(\mathbf{r})=\iint\left[g\left(\mathbf{r} \mid \mathbf{r}_{0}\right) \frac{\partial}{\partial n_{0}} p\left(\mathbf{r}_{0}\right)-p\left(\mathbf{r}_{0}\right) \frac{\partial}{\partial n_{0}} g\left(\mathbf{r} \mid \mathbf{r}_{0}\right)\right] d S_{0}+\iiint g\left(\mathbf{r} \mid \mathbf{r}_{0}\right) f\left(\mathbf{r}_{0}\right) d V_{0} .
$$

Up to this point, there have been no assumptions as to the nature of the pressure wave. What is desired is to predict the total pressure, $p_{t}(\mathbf{r})$, which is the sum of a known incident pressure, $p_{i}(\mathbf{r})$, and a scattered pressure from an inhomogeneity. The total pressure at any position $\mathbf{r}$, is

$$
p_{t}(\mathbf{r})=\iint\left[g\left(\mathbf{r} \mid \mathbf{r}_{0}\right) \frac{\partial}{\partial n_{0}} p_{t}\left(\mathbf{r}_{0}\right)-p_{t}\left(\mathbf{r}_{0}\right) \frac{\partial}{\partial n_{0}} g\left(\mathbf{r} \mid \mathbf{r}_{0}\right)\right] d S_{0}+\iiint g\left(\mathbf{r} \mid \mathbf{r}_{0}\right) f_{s}\left(\mathbf{r}_{0}\right) d V_{0},
$$


where,

$$
f_{s}\left(\mathbf{r}_{0}\right)=-\left\{\nabla_{0} \bullet\left[\gamma_{\rho}\left(\mathbf{r}_{0}\right) \nabla_{0} p_{t}\left(\mathbf{r}_{0}\right)\right]-\gamma_{\kappa}\left(\mathbf{r}_{0}\right) k_{0}^{2} p_{t}\left(\mathbf{r}_{0}\right)\right\} .
$$

The total pressure is represented by the pressure wave on the surface bounding the medium (the surface integral) and the pressure wave within the volume of the bounding medium (the volume integral). The Green's function for Eq. (2.41) is for an unbounded medium, therefore the surface integral is over a boundary at infinity. At infinity, the only contribution to the total pressure wave is from the incident wave, $p_{i}(\mathbf{r})$, since any components from the volume scatterer would go to zero due to spherical spreading as the distance approached infinity. This implies that the incident pressure wave can be substituted for the surface integral [17],

$$
p_{i}(\mathbf{r})=\iint\left[g\left(\mathbf{r} \mid \mathbf{r}_{0}\right) \frac{\partial}{\partial n_{0}} p_{t}\left(\mathbf{r}_{0}\right)-p_{t}\left(\mathbf{r}_{0}\right) \frac{\partial}{\partial n_{0}} g\left(\mathbf{r} \mid \mathbf{r}_{0}\right)\right] d S_{0} .
$$

Substituting Eqs. (2.57) and (2.58) into Eq. (2.56):

$$
p_{t}(\mathbf{r})=p_{i}(\mathbf{r})+\iiint\left\{\gamma_{\kappa}\left(\mathbf{r}_{0}\right) k_{0}^{2} p_{t}\left(\mathbf{r}_{0}\right)-\nabla_{0} \bullet\left[\gamma_{\rho}\left(\mathbf{r}_{0}\right) \nabla_{0} p_{t}\left(\mathbf{r}_{0}\right)\right]\right\} g\left(\mathbf{r} \mid \mathbf{r}_{0}\right) d V_{0} .
$$

Distributing the Green's function and separating the integrand gives

$$
p_{t}(\mathbf{r})=p_{i}(\mathbf{r})+\iiint k_{0}^{2} \gamma_{\kappa}\left(\mathbf{r}_{0}\right) g\left(\mathbf{r} \mid \mathbf{r}_{0}\right) p_{t}\left(\mathbf{r}_{0}\right) d V_{0}-\iiint g\left(\mathbf{r} \mid \mathbf{r}_{0}\right) \nabla_{0} \bullet\left[\gamma_{\rho}\left(\mathbf{r}_{0}\right) \nabla_{0} p_{t}\left(\mathbf{r}_{0}\right)\right] d V_{0} .
$$

The second volume integral can be written as the difference of two volume integrals:

$$
\begin{aligned}
& \iiint_{g}\left(\mathbf{r} \mid \mathbf{r}_{0}\right) \nabla_{0} \bullet\left[\gamma_{\rho}\left(\mathbf{r}_{0}\right) \nabla_{0} p_{t}\left(\mathbf{r}_{0}\right)\right] d V_{0} \\
& =\iiint \nabla_{0} \bullet\left[\gamma_{\rho}\left(\mathbf{r}_{0}\right) g\left(\mathbf{r} \mid \mathbf{r}_{0}\right) \nabla_{0} p_{t}\left(\mathbf{r}_{0}\right)\right] d V_{0}-\iiint \gamma_{\rho}\left(\mathbf{r}_{0}\right) \nabla_{0} p_{t}\left(\mathbf{r}_{0}\right) \nabla_{0} g\left(\mathbf{r} \mid \mathbf{r}_{0}\right) d V_{0} .
\end{aligned}
$$

Using the divergence theorem, the first volume integral on the right hand side can be changed into a surface integral [18]:

$$
\iiint \nabla_{0} \bullet\left[\gamma_{\rho}\left(\mathbf{r}_{0}\right) g\left(\mathbf{r} \mid \mathbf{r}_{0}\right) \nabla_{0} p_{t}\left(\mathbf{r}_{0}\right)\right] d V_{0}=\iint \frac{\partial}{\partial n_{0}}\left[\gamma_{\rho}\left(\mathbf{r}_{0}\right) g\left(\mathbf{r} \mid \mathbf{r}_{0}\right) \nabla_{0} p_{t}\left(\mathbf{r}_{0}\right)\right] d S_{0} \cdot
$$

On the surface, the density factor $\left(\gamma_{\rho}\right)$ is assumed to go to zero [Eq. (2.36)] [16], so

$$
\iint \frac{\partial}{\partial n_{0}}\left[\gamma_{\rho}\left(\mathbf{r}_{0}\right) g\left(\mathbf{r} \mid \mathbf{r}_{0}\right) \nabla_{0} p_{t}\left(\mathbf{r}_{0}\right)\right] d S_{0}=0 .
$$


This allows the total pressure wave, Eq. (2.59), to be written:

$$
p_{t}(\mathbf{r})=p_{i}(\mathbf{r})+\iiint\left[k_{0}^{2} \gamma_{\kappa}\left(\mathbf{r}_{0}\right) g\left(\mathbf{r} \mid \mathbf{r}_{0}\right) p_{t}\left(\mathbf{r}_{0}\right)+\gamma_{\rho}\left(\mathbf{r}_{0}\right) \nabla_{0} p_{t}\left(\mathbf{r}_{0}\right) \nabla_{0} g\left(\mathbf{r} \mid \mathbf{r}_{0}\right)\right] d V_{0} .
$$

\subsection{Backscatter From a Weak Scatterer}

\subsubsection{Born Approximation}

Within the discrete volume, $V_{u}$, the density $\left(\gamma_{\rho}\right)$ and compressibility $\left(\gamma_{\kappa}\right)$ factors are constant. Outside the discrete volume, the factors are equal to zero. This means that outside of the volume $\left(V_{a}\right)$, the integrand of the volume integral in Eq. (2.64) is zero, and within the volume: $\gamma_{\rho}, \gamma_{\kappa}=$ constants. The total pressure wave can now be written:

$$
p_{t}(\mathbf{r})=p_{i}(\mathbf{r})+\iiint_{V_{u}}\left[k_{m}^{2} \gamma_{\kappa} g\left(\mathbf{r} \mid \mathbf{r}_{0}\right) p_{t}\left(\mathbf{r}_{0}\right)+\gamma_{\rho} \nabla_{0} p_{t}\left(\mathbf{r}_{0}\right) \nabla_{0} g\left(\mathbf{r} \mid \mathbf{r}_{0}\right)\right] d V_{0} .
$$

In the above equation, the subscript " 0 " for the wavenumber has been change to " $m$ " for the homogeneous medium. This is to avoid confusion with the subscript " 0 " used to define the reference coordinate system within the discrete inhomogeneity. This convention will be used in the remainder of this thesis.

If the scattered wave from the discrete inhomogeneity is not very intense, then as an approximation, the total pressure terms, $p_{t}(\mathbf{r})$, within the integrand can be replaced with the incident pressure terms, $p_{i}(\mathbf{r})$. This is called the Born approximation [16]. For this study, the speed of sound contrast, $c_{a} / c_{m}$, and density contrast, $\rho_{a} / \rho_{m}$, are $[19,20]$ :

$$
\begin{aligned}
& c_{a} / c_{m}=1.028 \\
& \rho_{a} / \rho_{m}=1.036 .
\end{aligned}
$$

The density and compressibility factors are related to the sound speed and density contrasts by the following relationships: 


$$
\begin{gathered}
\gamma_{\kappa}=\frac{1-\left(\rho_{a} / \rho_{m}\right)\left(c_{a} / c_{m}\right)^{2}}{\left(\rho_{a} / \rho_{m}\right)\left(c_{a} / c_{m}\right)^{2}} \\
\gamma_{\rho}=\frac{\rho_{a} / \rho_{m}-1}{\rho_{a} / \rho_{m}} .
\end{gathered}
$$

Substituting Eq. (2.66) into Eq. (2.67), the compressibility and density factors are found to be:

$$
\begin{aligned}
& \gamma_{\kappa}=-0.086 \\
& \gamma_{\rho}=+0.035 .
\end{aligned}
$$

These factors are small enough to consider the discrete inhomogeneity to be a "weak" scatterer, making the Born approximation applicable. Applying the Born approximation to Eq. (2.65) yields:

$$
p_{t}(\mathbf{r})=p_{i}(\mathbf{r})+\iiint_{V_{a}}\left[k_{m}^{2} \gamma_{\kappa} g\left(\mathbf{r} \mid \mathbf{r}_{0}\right) p_{i}\left(\mathbf{r}_{0}\right)+\gamma_{\rho} \nabla_{0} p_{i}\left(\mathbf{r}_{0}\right) \nabla_{0} g\left(\mathbf{r} \mid \mathbf{r}_{0}\right)\right] d V_{0} .
$$

The total pressure wave is determined to be the sum of the incident pressure wave, and the radiated pressure wave produced from the incident wave scattering from the discrete inhomogeneity. Thus, if one can describe the pressure field within the volume, $V_{a}$, the pressure anywhere outside the volume can be determined. The Born approximation allows the radiated pressure to be calculated in terms of the incident pressure wave, simplifying the determination of the pressure field within the discrete inhomogeneity. 


\subsubsection{Green's Function and Incident Pressure Wave}

For an unbounded medium, at large distances from the scattering volume, $V_{a}$, the Green's function is [17]:

$$
g\left(\mathbf{r} \mid \mathbf{r}_{0}\right)=\frac{1}{4 \pi\left|\mathbf{r}-\mathbf{r}_{0}\right|} e^{i\left(k_{m r} r-\mathbf{k}_{s} \cdot \mathbf{r}_{0}\right)} .
$$

\section{PROBLEM GEOMETRY II}

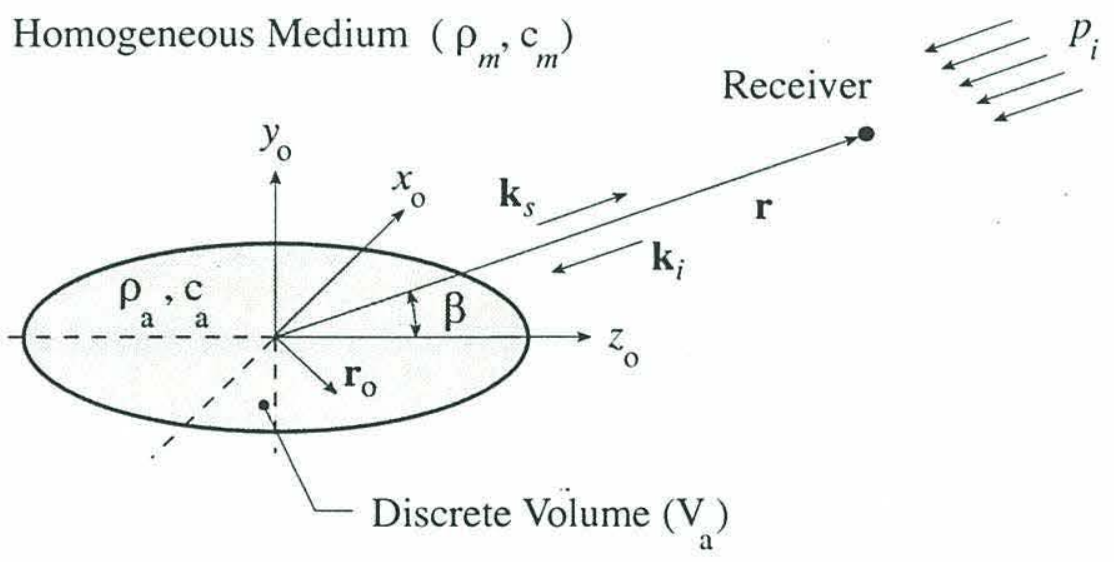

Figure 2-4: Geometry for the Green's function, the incident pressure wave, and the angle between the discrete volume's major axis and the receiver (the orientation angle), $b$.

Here, the position vectors ( $\mathbf{r}$ and $\mathbf{r}_{0}$ ) are defined in Eq. (2.42) and Fig. 2-4. The scattered wavenumber $\left(\mathbf{k}_{s}\right)$ is a vector pointing in the same direction as the position vector from the discrete inhomogeneity to the receiver (point of observation). The distance from the scatterer to the receiver is much greater than the size of the scatterer, so

$$
|\mathbf{r}| \gg\left|\mathbf{r}_{0}\right| \text {, }
$$

thus,

$$
|\mathbf{r}| \approx\left|\mathbf{r}-\mathbf{r}_{0}\right|
$$


Substituting Eq. (2.72) into Eq. (2.70):

$$
\begin{aligned}
g\left(\mathbf{r} \mid \mathbf{r}_{0}\right) & =\frac{1}{4 \pi|\mathbf{r}|} e^{i\left(k_{m r} r-k_{i} \cdot \mathbf{r}_{0}\right)} \\
& =\frac{1}{4 \pi r} e^{i k_{m} r} e^{-i \mathbf{k}_{s} \cdot \mathbf{r}_{0}} .
\end{aligned}
$$

To solve Eq. (2.69), the gradient of the Green's function [Eq. (2.73)] is also needed [18]:

$$
\begin{aligned}
\nabla_{0} g\left(\mathbf{r} \mid \mathbf{r}_{0}\right) & =\nabla_{0}\left(\frac{1}{4 \pi r} e^{i k_{m} r} e^{-i \mathbf{k}_{s} \cdot \mathbf{r}_{0}}\right) \\
& =\frac{1}{4 \pi r} e^{i k_{m} r} \nabla_{0}\left(e^{-i \mathbf{k}_{s} \cdot \mathbf{r}_{0}}\right) \\
& =\frac{i}{4 \pi r} e^{i\left(k_{m r} r-k_{s} \cdot \mathbf{r}_{0}\right)} \mathbf{k}_{s} .
\end{aligned}
$$

The distance from the source producing the incident pressure wave to the discrete scatterer is sufficient $(k r \gg 1)$ to allow the incident pressure to be considered a plane wave of the form

$$
p_{i}\left(\mathbf{r}_{0}\right)=A e^{i \mathbf{k}_{i} \cdot \mathbf{r}_{0}} .
$$

The gradient of the incident pressure wave is

$$
\begin{aligned}
\nabla_{0} p_{i}\left(\mathbf{r}_{0}\right) & =\nabla_{0}\left(A e^{i \mathbf{k}_{i} \cdot \mathbf{r}_{0}}\right) \\
& =A \nabla_{0}\left(e^{i \mathbf{k}_{i} \cdot \mathbf{r}_{0}}\right) \\
& =i A e^{i \mathbf{k}_{i} \cdot \mathbf{r}_{0}} \mathbf{k}_{i} .
\end{aligned}
$$

Now, defining the scattered pressure radiated from the discrete volume as:

$$
p_{s}(\mathbf{r})=\iiint_{V_{a}}\left[k_{m}^{2} \gamma_{\kappa} g\left(\mathbf{r} \mid \mathbf{r}_{0}\right) p_{i}\left(\mathbf{r}_{0}\right)+\gamma_{\rho} \nabla_{0} p_{i}\left(\mathbf{r}_{0}\right) \nabla_{0} g\left(\mathbf{r} \mid \mathbf{r}_{0}\right)\right] d V_{0} .
$$

Substituting Eqs. (2.73), (2.74), (2.75), and (2.76) into Eq. (2.77),

$$
\begin{aligned}
p_{s}(\mathbf{r}) & =\iiint_{V_{a}}\left[k_{m}^{2} \gamma_{\kappa}\left(\frac{1}{4 \pi r} e^{i\left(k_{m} r-\mathbf{k}_{s} \cdot \mathbf{r}_{0}\right)}\right) \cdot\left(A e^{i \mathbf{k}_{i} \cdot \mathbf{r}_{0}}\right)+\gamma_{\rho}\left(i A e^{i \mathbf{k}_{i} \cdot \boldsymbol{r}_{i n}} \mathbf{k}_{i}\right) \cdot\left(\frac{i}{4 \pi r} e^{i\left(k_{m} r-\mathbf{k}_{s} \cdot \mathbf{r}_{n}\right)} \mathbf{k}_{s}\right)\right] d V_{0} \\
& =\frac{A e^{i k_{m} r}}{4 \pi r} \iiint_{V_{a}}\left(k_{m}^{2} \gamma_{\kappa} e^{i\left(\mathbf{k}_{i} \cdot \mathbf{r}_{0}-\mathbf{k}_{s} \cdot \mathbf{r}_{0}\right)}-\gamma_{\rho} \mathbf{k}_{s} \bullet \mathbf{k}_{i} e^{i\left(\mathbf{k}_{s} \cdot \mathbf{r}_{0}-\mathbf{k}_{s} \cdot \mathbf{r}_{0}\right)}\right) d V_{0} \\
& =\frac{A e^{i k_{m} r}}{4 \pi r} \iiint_{V_{a}}\left(\gamma_{\kappa} k_{m}^{2}-\gamma_{\rho} \mathbf{k}_{s} \bullet \mathbf{k}_{i}\right) e^{i\left(\mathbf{k}_{i}-\mathbf{k}_{s}\right) \cdot \mathbf{r}_{0}} d V_{0}
\end{aligned}
$$


From Fig. 2-4, it can be seen that

$$
\mathbf{k}_{s}=-\mathbf{k}_{i} \text {. }
$$

The magnitude of the wavenumber is:

$$
\left|\mathbf{k}_{s}\right|=\left|\mathbf{k}_{i}\right|=k_{m}=\left(c_{a} / c_{0}\right) k_{a} \equiv h k_{a} .
$$

Thus,

$$
\mathbf{k}_{s} \bullet \mathbf{k}_{i}=k_{m}^{2},
$$

and

$$
\mathbf{k}_{i}-\mathbf{k}_{s}=2 \mathbf{k}_{i} .
$$

Using Eqs. (2.81) and (2.82), Eq. (2.78) reduces to

$$
p_{s}(\mathbf{r})=\frac{A k_{m}^{2} e^{i k_{m} r}}{4 \pi r}\left(\gamma_{\kappa}-\gamma_{\rho}\right) \iint_{V_{a}} e^{i 2 \mathbf{k}_{i} \cdot \boldsymbol{r}_{0}} d V_{0} .
$$

For a weak scattering, discrete inhomogeneity within a homogeneous medium, the backscattered pressure radiating from the volume is a function of the amplitude of the incident pressure wave, the range from the discrete volume to the receiver, and the shape of the discrete volume. To remove the effects of range and incident pressure amplitude, a function known as the backscatter amplitude $\left[\Phi\left(\mathbf{e}_{i}\right)\right]$ is defined in the following formula [21]:

$$
p_{s}(\mathbf{r})=\frac{A e^{i k_{m} r}}{r} \Phi\left(\mathbf{e}_{i}\right) \text {. }
$$

Thus, the backscatter amplitude is equal to:

$$
\Phi\left(\mathbf{e}_{i}\right)=\frac{k_{m}^{2}}{4 \pi}\left(\gamma_{\kappa}-\gamma_{\rho}\right) \iint_{V_{a}} e^{i 2 \mathbf{k}_{i} \cdot \boldsymbol{r}_{0}} d V_{0} .
$$

This shows that the backscatter amplitude is a function of the material properties and shape of the inhomogeneity, and the direction of the incident wave $\left(\mathbf{e}_{i}\right)$. 


\subsection{Prolate Spheroid Geometry}

\subsubsection{Shape of Model and the Definition of Integration Limits}

The geometry of the prolate spheroid is integrated using the cylindrical coordinates $\left(\xi_{0}, z_{0}, \theta_{0}\right)$, defined in Fig. 2-5. The orientation angle, $\beta$, defines the direction of the incident wave with respect to the prolate spheroid, $\mathbf{e}_{i}$, (Fig. 2-4). The backscatter amplitude can be written:

$$
\Phi(\beta)=\frac{k_{m}^{2}}{4 \pi}\left(\gamma_{\kappa}-\gamma_{\rho}\right) \int_{-L / 2}^{L / 2} \int_{0}^{\xi\left(z_{0}\right)_{2 \pi}^{2 \pi}} \int_{0}^{i 2 \mathbf{k}_{i} \cdot \mathbf{r}_{0}} d \theta_{0} \xi_{0} d \xi_{0} d z_{0} .
$$

Where, for a prolate spheroid,

$$
\xi\left(z_{0}\right)=\frac{a}{L} \sqrt{L^{2}-4 z_{0}^{2}}
$$

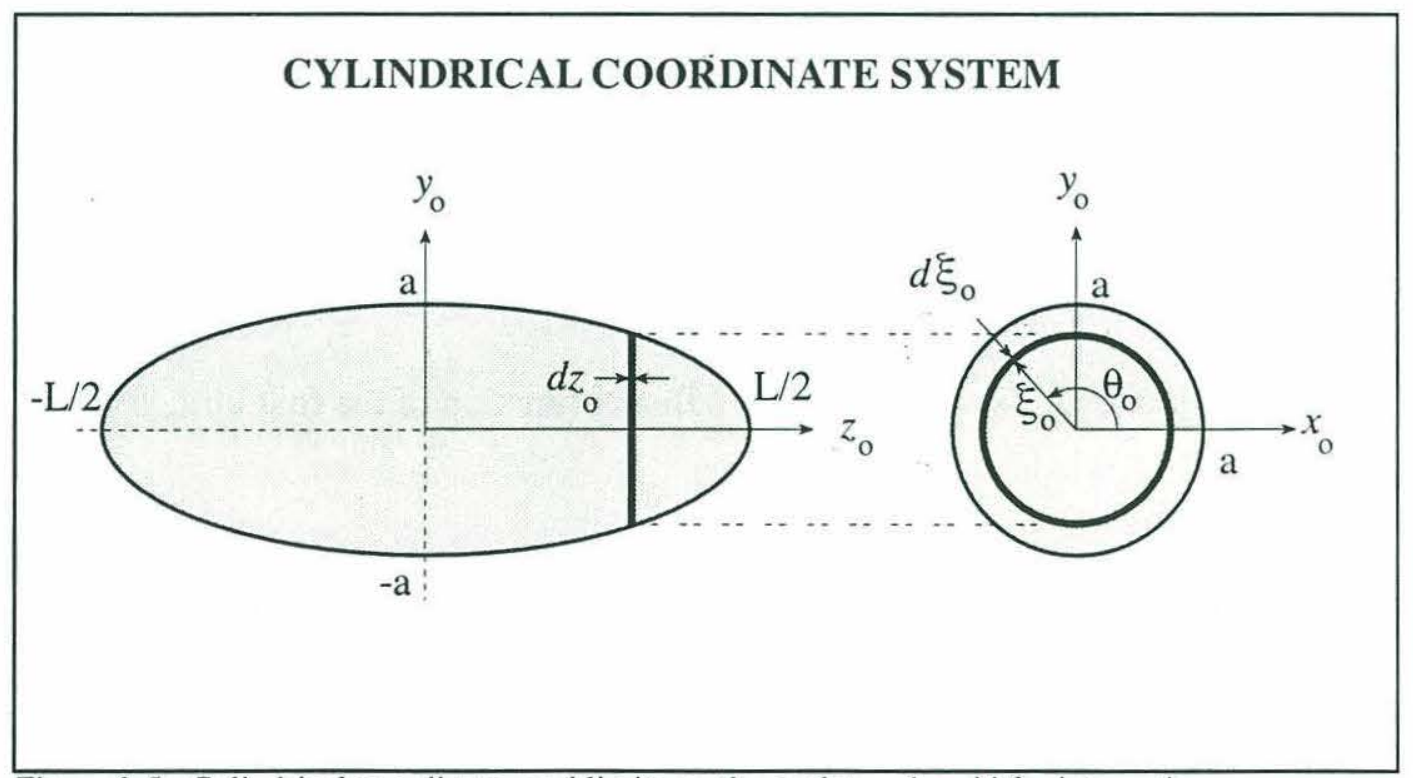

Figure 2-5: Cylindrical coordinates and limits on the prolate spheroid for integration. 
The vectors $\mathbf{k}_{i}$ and $\mathbf{r}_{0}$ can be written in terms of the orientation angle and cylindrical coordinates defined in Figs. 2-4 and 2-5:

$$
\mathbf{k}_{i}=-k_{a}\left[\cos (\beta) \mathbf{e}_{z_{0}}+\sin (\beta) \mathbf{e}_{x_{0}}\right]
$$

and,

$$
\mathbf{r}_{0}=\xi_{0} \cos \left(\theta_{0}\right) \mathbf{e}_{x_{0}}+\xi_{0} \sin \left(\theta_{0}\right) \mathbf{e}_{y_{0}}+z_{0} \mathbf{e}_{z_{0}}
$$

Within the volume to be integrated, the material properties of the discrete inhomogeneity are used, instead of the material properties of the homogeneous medium. This is referred to as the distorted wave Born approximation, in which the internal wave is assumed to be the same as the incident wave, but is corrected for the material conditions that the internal wave is exposed to.

The exponent in the integrand of Eq. (2.86) can be written:

$$
i 2 \mathbf{k}_{i} \bullet \mathbf{r}_{0}=-i 2 k_{a}\left[z_{0} \cos (\beta)+\xi_{0} \cos \left(\theta_{0}\right) \sin (\beta)\right] .
$$

Applying Eq. (2.90) to Eq. (2.86):

$$
\Phi(\beta)=\frac{k_{m}^{2}}{4 \pi}\left(\gamma_{\kappa}-\gamma_{\rho}\right) \int_{-L / 2}^{L / 2} e^{-i 2 k_{u} z_{0} \cos (\beta)} \int_{0}^{\xi\left(z_{0}\right)} \xi_{0} \int_{0}^{2 \pi} e^{-i 2 k_{a} \xi_{0} \cos \left(\theta_{0}\right) \sin (\beta)} d \theta_{0} d \xi_{0} d z_{0} .
$$

\subsubsection{Solution of the Integral for the Prolate Spheroid Geometry}

The integral over the variable $\theta_{0}$, is a Bessel function of the first kind, of order zero [22]:

$$
\begin{aligned}
\Phi(\beta) & =\frac{k_{m}^{2}}{4 \pi}\left(\gamma_{\kappa}-\gamma_{\rho}\right) \int_{-L / 2}^{L / 2} e^{-i 2 k_{a} z_{0} \cos (\beta)} \int_{0}^{\xi\left(z_{0}\right)} \xi_{0}\left\{2 \pi \mathrm{J}_{0}\left[2 k_{a} \xi_{0} \sin (\beta)\right]\right\} d \xi_{0} d z_{0} \\
& =\frac{k_{m}^{2}}{2}\left(\gamma_{\kappa}-\gamma_{\rho}\right) \int_{-L / 2}^{L / 2} e^{-i 2 k_{a} z_{0} \cos (\beta)} \int_{0}^{\xi\left(z_{0}\right)} \xi_{0} \mathrm{~J}_{0}\left[2 k_{a} \xi_{0} \sin (\beta)\right] d \xi_{0} d z_{0} .
\end{aligned}
$$


To integrate over the variable $\xi_{0}$, the integration variable is changed to $\eta_{0}=2 k_{a} \xi_{0} \sin (\beta)$ :

$$
\begin{aligned}
\Phi(\beta) & =\frac{k_{m}^{2}}{2}\left(\gamma_{\kappa}-\gamma_{\rho}\right) \int_{-L / 2}^{L / 2} e^{-i 2 k_{u} z_{0} \cos (\beta)} \int_{0}^{\eta\left(z_{0}\right)} \frac{\left[2 k_{a} \xi_{0} \sin (\beta)\right]}{\left[2 k_{a} \sin (\beta)\right]^{2}} \mathrm{~J}_{0}\left[2 k_{a} \xi_{0} \sin (\beta)\right] d\left[2 k_{a} \xi_{0} \sin (\beta)\right] d z_{0} \\
& =\frac{k_{m}^{2}}{8 k_{a}^{2} \sin ^{2}(\beta)}\left(\gamma_{\kappa}-\gamma_{\rho}\right) \int_{-L / 2}^{L / 2} e^{-i 2 k_{a} z_{0} \cos (\beta)} \int_{0}^{\eta\left(z_{0}\right)} \eta_{0} \mathrm{~J}_{0}\left(\eta_{0}\right) d \eta_{0} d z_{0}
\end{aligned}
$$

Here,

$$
\eta\left(z_{0}\right)=2 k_{a} \sin (\beta) \frac{a}{L} \sqrt{L^{2}-4 z_{0}^{2}} .
$$

The integral over the variable $\eta_{0}$ produces a Bessel function of the first kind, of order one [23]:

$$
\Phi(\beta)=\frac{k_{m}^{2}}{8 k_{a}^{2} \sin ^{2}(\beta)}\left(\gamma_{\kappa}-\gamma_{\rho}\right) \int_{-L / 2}^{L / 2} e^{-i 2 k_{u} z_{0} \cos (\beta)}\left\{\eta\left(z_{0}\right) J_{1}\left[\eta\left(z_{0}\right)\right]\right\} d z_{0} .
$$

Substituting Eq. (2.94) into Eq. (2.95), and simplifying:

$$
\begin{aligned}
\Phi(\beta) & =\frac{k_{m}^{2}\left(\gamma_{\kappa}-\gamma_{\rho}\right)}{8 k_{a}^{2} \sin ^{2}(\beta)} \int_{-L / 2}^{L / 2} e^{-i 2 k_{a} z_{0} \cos (\beta)}\left\{2 k_{a} \sin (\beta) \frac{a}{L} \sqrt{L^{2}-4 z_{0}^{2}} \mathrm{~J}_{1}\left[2 k_{a} \sin (\beta) \frac{a}{L} \sqrt{L^{2}-4 z_{0}^{2}}\right]\right\} d z_{0} \\
& =\frac{k_{m}^{2} a\left(\gamma_{\kappa}-\gamma_{\rho}\right)}{2 k_{a} L \sin (\beta)} \int_{-L / 2}^{L / 2} e^{-i 2 k_{u} \cos (\beta) z_{0}} \sqrt{\left(\frac{L}{2}\right)^{2}-z_{0}^{2}} \mathrm{~J}_{1}\left[\frac{4 k_{a} a \sin (\beta)}{L} \sqrt{\left(\frac{L}{2}\right)^{2}-z_{0}^{2}}\right] d z_{0} .
\end{aligned}
$$

To perform the final integration, the exponential in the integrand must be changed to a sine and cosine representation:

$$
e^{-i 2 k_{a} \cos (\beta) z_{0}}=\cos \left(\mu z_{0}\right)-i \sin \left(\mu z_{0}\right),
$$

with $\mu=2 k_{a} \cos (\beta)$. Defining for simplicity,

$$
C_{\Phi}=\frac{k_{m}^{2} a\left(\gamma_{\kappa}-\gamma_{\rho}\right)}{2 k_{a} L \sin (\beta)}
$$

and

$$
F\left(z_{0}\right)=\sqrt{\left(\frac{L}{2}\right)^{2}-z_{0}^{2}} \mathrm{~J}_{1}\left[\frac{4 k_{a} a \sin (\beta)}{L} \sqrt{\left(\frac{L}{2}\right)^{2}-z_{0}^{2}}\right],
$$


Eq. (2.96) can be written:

$$
\Phi(\beta)=C_{\Phi} \int_{-L / 2}^{L / 2} F\left(z_{0}\right)\left[\cos \left(\mu z_{0}\right)-i \sin \left(\mu z_{0}\right)\right] d z_{0} .
$$

This equation can be reduced to a simpler form:

$$
\begin{aligned}
\Phi(\beta) & =C_{\Phi}\left\{\int_{-L / 2}^{0} F\left(z_{0}\right)\left[\cos \left(\mu z_{0}\right)-i \sin \left(\mu z_{0}\right)\right] d z_{0}+\int_{0}^{L / 2} F\left(z_{0}\right)\left[\cos \left(\mu z_{0}\right)-i \sin \left(\mu z_{0}\right)\right] d z_{0}\right\} \\
& =C_{\Phi}\left\{-\int_{0}^{L / 2} F\left(z_{0}\right)\left[\cos \left(\mu z_{0}\right)-i \sin \left(\mu z_{0}\right)\right] d z_{0}+\int_{0}^{L / 2} F\left(z_{0}\right)\left[\cos \left(\mu z_{0}\right)-i \sin \left(\mu z_{0}\right)\right] d z_{0}\right\} \\
& =C_{\Phi}\left\{-\int_{0}^{L / 2} F\left(-z_{0}\right)\left[\cos \left(-\mu z_{0}\right)-i \sin \left(-\mu z_{0}\right)\right]\left(-d z_{0}\right)+\int_{0}^{L / 2} F\left(z_{0}\right)\left[\cos \left(\mu z_{0}\right)-i \sin \left(\mu z_{0}\right)\right] d z_{0}\right\} \\
& =C_{\Phi}\left\{\int_{0}^{L / 2} F\left(z_{0}\right)\left[\cos \left(\mu z_{0}\right)+i \sin \left(\mu z_{0}\right)\right] d z_{0}+\int_{0}^{L / 2} F\left(z_{0}\right)\left[\cos \left(\mu z_{0}\right)-i \sin \left(\mu z_{0}\right)\right] d z_{0}\right\} \\
& =2 C_{\Phi} \int_{0}^{L / 2} F\left(z_{0}\right) \cos \left(\mu z_{0}\right) d z_{0} .
\end{aligned}
$$

The fact that $F\left(z_{0}\right)=F\left(-z_{0}\right)$ is used in deriving Eq. (2.101). Substituting Eqs. (2.98) and (2.99) into Eq. (2.101):

$$
\Phi(\beta)=\frac{k_{m}^{2} a\left(\gamma_{\kappa}-\gamma_{\rho}\right)}{k_{a} L \sin (\beta)} \int_{0}^{L / 2} \sqrt{\left(\frac{L}{2}\right)^{2}-z_{0}^{2}} \mathrm{~J}_{1}\left[\frac{4 k_{a} a \sin (\beta)}{L} \sqrt{\left(\frac{L}{2}\right)^{2}-z_{0}^{2}}\right] \cos \left[2 k_{a} \cos (\beta) z_{0}\right] d z_{0} .
$$

Integrating over the variable $z_{0}[24]$ :

$$
\begin{aligned}
\Phi(\beta)= & \frac{k_{m}^{2} a\left(\gamma_{\kappa}-\gamma_{\rho}\right)}{k_{a} L \sin (\beta)} \sqrt{\frac{\pi}{2}}\left(\frac{L}{2}\right)^{3 / 2} \frac{4 k_{a} a \sin (\beta)}{L}\left\{\left[\frac{4 k_{a} a \sin (\beta)}{L}\right]^{2}+\left[2 k_{a} \cos (\beta)\right]^{2}\right\}^{-3 / 4} \\
& \times \mathrm{J}_{3 / 2}\left\{\frac{L}{2} \sqrt{\left.\left[\frac{4 k_{a} a \sin (\beta)}{L}\right]^{2}+\left[2 k_{a} \cos (\beta)\right]^{2}\right\}}\right. \\
= & \frac{k_{m}^{2} a^{2} L\left(\gamma_{\kappa}-\gamma_{\rho}\right) \sqrt{\pi}}{2 \sqrt{2}} \frac{\mathrm{J}_{3 / 2}\left[k_{a} \sqrt{4 a^{2} \sin ^{2}(\beta)+L^{2} \cos ^{2}(\beta)}\right]}{\left[k_{a} \sqrt{4 a^{2} \sin ^{2}(\beta)+L^{2} \cos ^{2}(\beta)}\right]^{3 / 2}}
\end{aligned}
$$


The cylindrical Bessel function $\mathrm{J}_{3 / 2}(\zeta)$, can be transformed into a spherical Bessel function $j_{1}(\zeta)$, by the following equation [25]:

$$
\mathrm{J}_{3 / 2}(\zeta)=j_{1}(\zeta) \sqrt{\frac{2 \zeta}{\pi}} .
$$

Applying Eq. (2.104) to Eq. (2.103):

$$
\begin{aligned}
\Phi(\beta) & =\frac{k_{m}^{2} a^{2} L\left(\gamma_{\kappa}-\gamma_{\rho}\right)}{2} \frac{j_{1}\left[k_{a} \sqrt{4 a^{2} \sin ^{2}(\beta)+L^{2} \cos ^{2}(\beta)}\right]}{\left[k_{a} \sqrt{4 a^{2} \sin ^{2}(\beta)+L^{2} \cos ^{2}(\beta)}\right]^{3 / 2}}\left[k_{a} \sqrt{4 a^{2} \sin ^{2}(\beta)+L^{2} \cos ^{2}(\beta)}\right]^{1 / 2} \\
& =\frac{k_{m}^{2} a^{2} L\left(\gamma_{\kappa}-\gamma_{\rho}\right)}{2} \frac{j_{1}\left[k_{a} \sqrt{4 a^{2} \sin ^{2}(\beta)+L^{2} \cos ^{2}(\beta)}\right]}{k_{a} \sqrt{4 a^{2} \sin ^{2}(\beta)+L^{2} \cos ^{2}(\beta)}} .
\end{aligned}
$$

The spherical Bessel function of the first kind of order one can be written as [25]:

$$
j_{1}(\zeta)=\frac{1}{\zeta}\left[\frac{\sin (\zeta)}{\zeta}-\cos (\zeta)\right] .
$$

Utilizing Eq. (2.106) to rewrite Eq. (2.105):

$$
\begin{aligned}
\Phi(\beta)= & \frac{k_{m}^{2} a^{2} L\left(\gamma_{\kappa}-\gamma_{\rho}\right)}{2 k_{a}^{2}\left[4 a^{2} \sin ^{2}(\beta)+L^{2} \cos ^{2}(\beta)\right]} \\
& \times\left\{\frac{\sin \left[k_{a} \sqrt{4 a^{2} \sin ^{2}(\beta)+L^{2} \cos ^{2}(\beta)}\right]}{k_{a} \sqrt{4 a^{2} \sin ^{2}(\beta)+L^{2} \cos ^{2}(\beta)}}-\cos \left[k_{a} \sqrt{4 a^{2} \sin ^{2}(\beta)+L^{2} \cos ^{2}(\beta)}\right]\right\} \\
= & \frac{h^{2} L\left(\gamma_{\kappa}-\gamma_{\rho}\right)}{8\left[\sin ^{2}(\beta)+\left(\frac{L}{2 a}\right)^{2} \cos ^{2}(\beta)\right]} \\
& \times\left\{\frac{\sin \left[\frac{2 k_{m} a}{h} \sqrt{\sin ^{2}(\beta)+\left(\frac{L}{2 a}\right)^{2} \cos ^{2}(\beta)}\right]}{\frac{2 k_{m} a}{h} \sqrt{\sin ^{2}(\beta)+\left(\frac{L}{2 a}\right)^{2} \cos ^{2}(\beta)}}-\cos \left[\frac{2 k_{m} a}{h} \sqrt{\sin ^{2}(\beta)+\left(\frac{L}{2 a}\right)^{2} \cos ^{2}(\beta)}\right]\right\} .
\end{aligned}
$$


This is the equation for the backscatter amplitude as a function of orientation angle. The equation can be written in a more compact form by defining,

$$
\psi(\beta) \equiv \frac{2}{h} \sqrt{\sin ^{2}(\beta)+\left(\frac{L}{2 a}\right)^{2} \cos ^{2}(\beta)} .
$$

Substituting Eq. (2.108) into Eq. (2.107):

$$
\Phi(\beta)=\frac{L\left(\gamma_{\kappa}-\gamma_{\rho}\right)}{4 \psi^{2}(\beta)}\left\{\frac{\sin \left[\left(k_{m} a\right) \psi(\beta)\right]}{\left(k_{m} a\right) \psi(\beta)}-\cos \left[\left(k_{m} a\right) \psi(\beta)\right]\right\} .
$$

A parameter called the differential backscatter cross section is defined as follows [21]:

$$
\sigma_{b s}(\beta)=|\Phi(\beta)|^{2}
$$

Thus, the differential backscatter cross section is:

$$
\sigma_{b s}(\beta)=\left|\frac{L\left(\gamma_{\kappa}-\gamma_{\rho}\right)}{4 \psi^{2}(\beta)}\left\{\frac{\sin \left[\left(k_{m} a\right) \psi(\beta)\right]}{\left(k_{m} a\right) \psi(\beta)}-\cos \left[\left(k_{m} a\right) \psi(\beta)\right]\right\}\right|^{2} .
$$

The backscatter cross section is related to another parameter, the backscatter target strength. Backscatter target strength is defined as $[26,27]$ :

$$
T S=10 \log \left[\frac{\sigma_{b s}(\beta)}{A_{r e f}}\right],
$$

where $A_{r e f}$ is a reference area used to make the argument of the logarithm dimensionless.

The cross section is seen to be a function of shape and material properties of the prolate spheroid as well as acoustic frequency. It is not solely a function of $k a$, as $\psi(\beta)$ is a function of $a$, but not a function of acoustic frequency (wavenumber). Nor is it a function of $L /(2 a)$ as the $[(k a) \psi(\beta)]$ terms are related to, but not sole functions of $L /(2 a)$. The cross section is characterized by $L$ and $a$. This is as expected. Both are used to define the shape of the spheroid, but the ratio of $L$ and $a$ is not sufficient, as the ratio does not account for the size of the inhomogeneity with respect to the acoustic wavelength. 


\subsection{Ideal Backscatter Directivity Pattern}

By plotting the equation for the differential backscatter cross section versus orientation angle [Eq. (2.111)], the backscatter directivity pattern is produced. The directivity patterns are normalized to the maximum backscatter cross section, since the shape of the curves and not their absolute amplitudes are being studied. Figure 2-6 shows normalized backscatter directivity patterns for various acoustic frequencies. The angular width of main (broadside) lobe of this plot is referred to as the "beamwidth" in this thesis (not to be confused with "beamwidth" of the radiation pattern of a transducer).

The beamwidth for this analysis is defined as the angle subtended between the smallest and largest angles at which the backscatter cross section reaches one-half the maximum value of the backscatter cross section. The beamwidth has been marked on Fig. 2-6 as a horizontal line at a normalized backscatter cross section of 0.5 .

Referring to Fig. 2-6, it is seen that the general trend is for the beamwidth of an individual lobe to get narrower with increasing frequency [Fig. 2-6 (a-c)]. Also, as the acoustic frequency is increased, the side lobes have a greater magnitude compared to the center lobe [Fig. 2-6 (d-g)]. This phenomenon can be understood by considering the prolate spheroid to be a three-dimensional array of infinitesimal point sources [28,29]. At certain frequencies, the geometry within the prolate spheroid would cause the pressure waves from the infinitesimal point sources to add out of phase, causing cancellation of pressure waves [30]. This produces nulls in the backscattering cross section at certain values of $k a[4,12,14]$, which in turn results in a splitting of the main lobe [Fig. 2-6 (h).] At higher frequencies, the null becomes narrower until the two side lobes "degenerate" into 

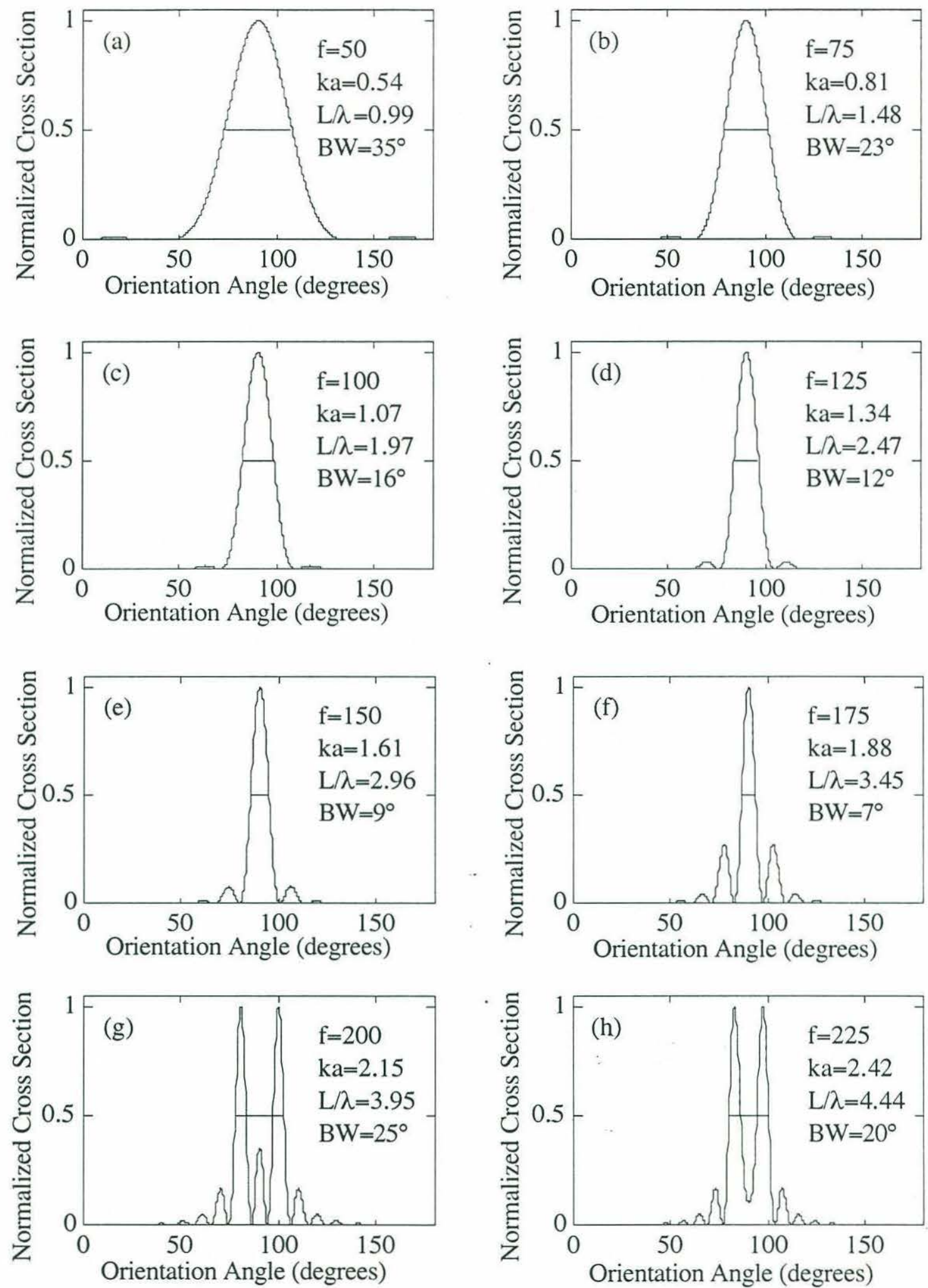

Figure 2-6: Normalized backscatter directivity patterns for a weak scattering prolate spheroid in a homogeneous medium. The nature of the shape changes in the directivity pattern made it necessary to define the beamwidth of the main lobe (BW) as the width of the pattern at the point where the backscatter cross section $\left(\sigma_{b s}\right)$ is one-half the maximum value. The beamwidth in each subplot is shown as a solid horizontal 

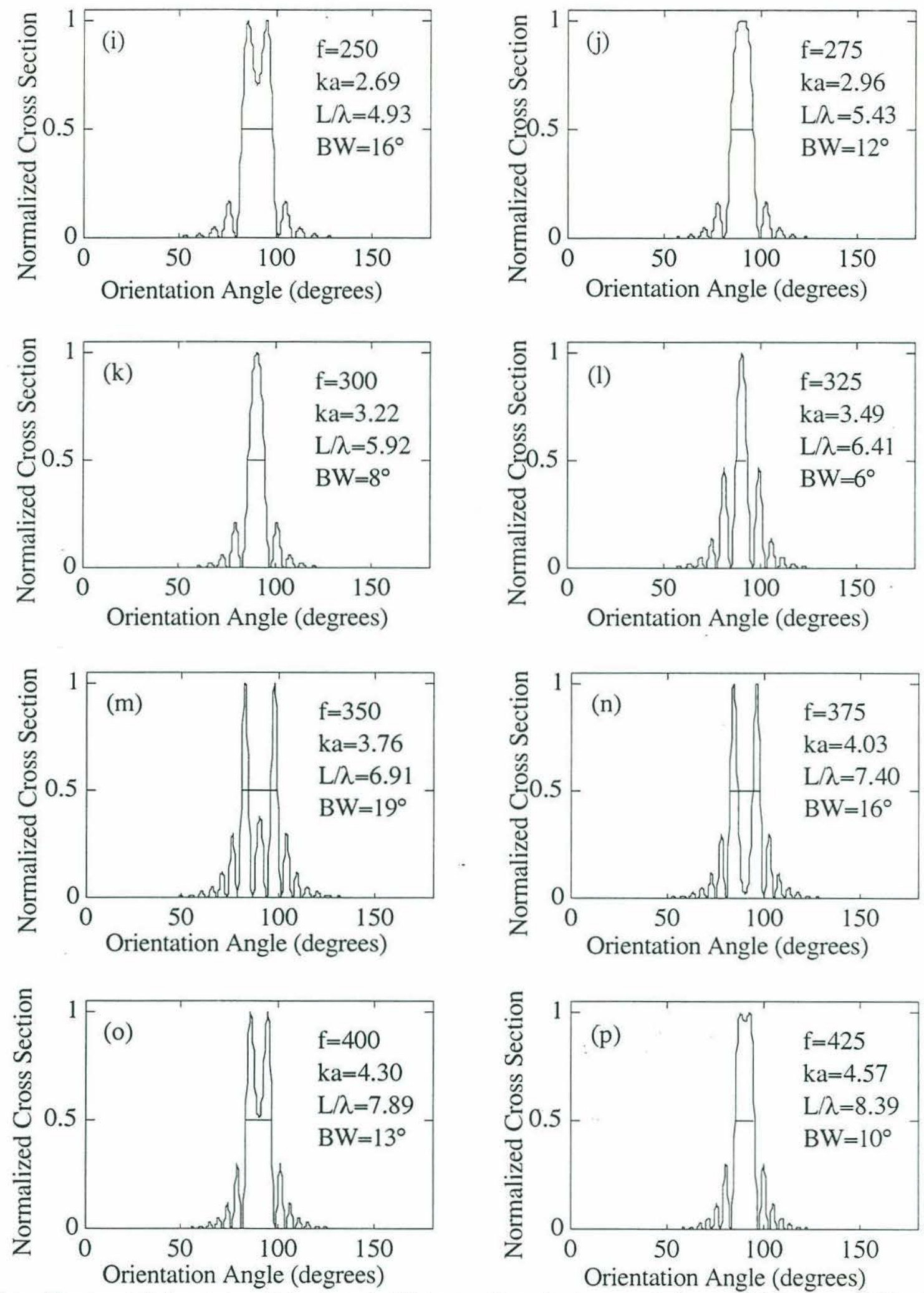

line. The acoustic frequency (f) is given in kilohertz. Note the increase in beamwidth between 175 and $200 \mathrm{kHz}$ and between 325 and $350 \mathrm{kHz}$. This increase is due to the method of defining the beamwidth. 
a new center lobe [Fig. 2-6 (i,j)]. The process repeats itself as frequency continues to increase [Fig. 2-6 (j-p)].

The phenomena described above result from the model for a decapod shrimp being a penetrable volume. Another model treats the animal as an impenetrable surface. The target strength equation for the impenetrable surface is:

$$
T S=10 \log \left[\frac{\sigma_{i p}(\beta)}{A_{r e f}}\right],
$$

where, the backscatter cross section for the impenetrable surface is [26]:

$$
\sigma_{i p}(\beta)=\frac{a^{2} \sin ^{2}[2 k L \cos (\beta)]}{4 \pi^{2} \cos ^{2}(\beta)} \sin ^{2}(\beta) .
$$

The backscatter directivity pattern based on the impenetrable boundary does not exhibit the same characteristics as the backscatter directivity pattern based upon the penetrable volume. At lower acoustic frequencies the patterns have similar shapes, with the prolate spheroid model exhibiting larger side lobes. At higher acoustic frequencies, the impenetrable plate has an increasingly narrow beamwidth, but the side lobe structure for the prolate spheroid gives the prolate spheroid's directivity pattern a wider beamwidth. Figure 2-7 compares the backscatter directivity patterns for the penetrable and the impenetrable cases.

Beamwidth can be plotted against a dimensionless parameter related to spheroid size and acoustic frequency. In Fig. 2-8, the beamwidth is plotted as a function of the frequency dependent dimensionless parameters $k a$ and $L / \lambda$. For each case the frequency is varied, but it is made non-dimensional by the radius of the prolate spheroid in the $k a$ case, and by the length of the prolate spheroid in the $L / \lambda$ case. The trends described above referring to Fig. 2-6 are evident in Fig. 2-8. Over the range of frequencies plotted (50 to 500 $\mathrm{kHz}$ ), three beamwidth step increases are seen. 

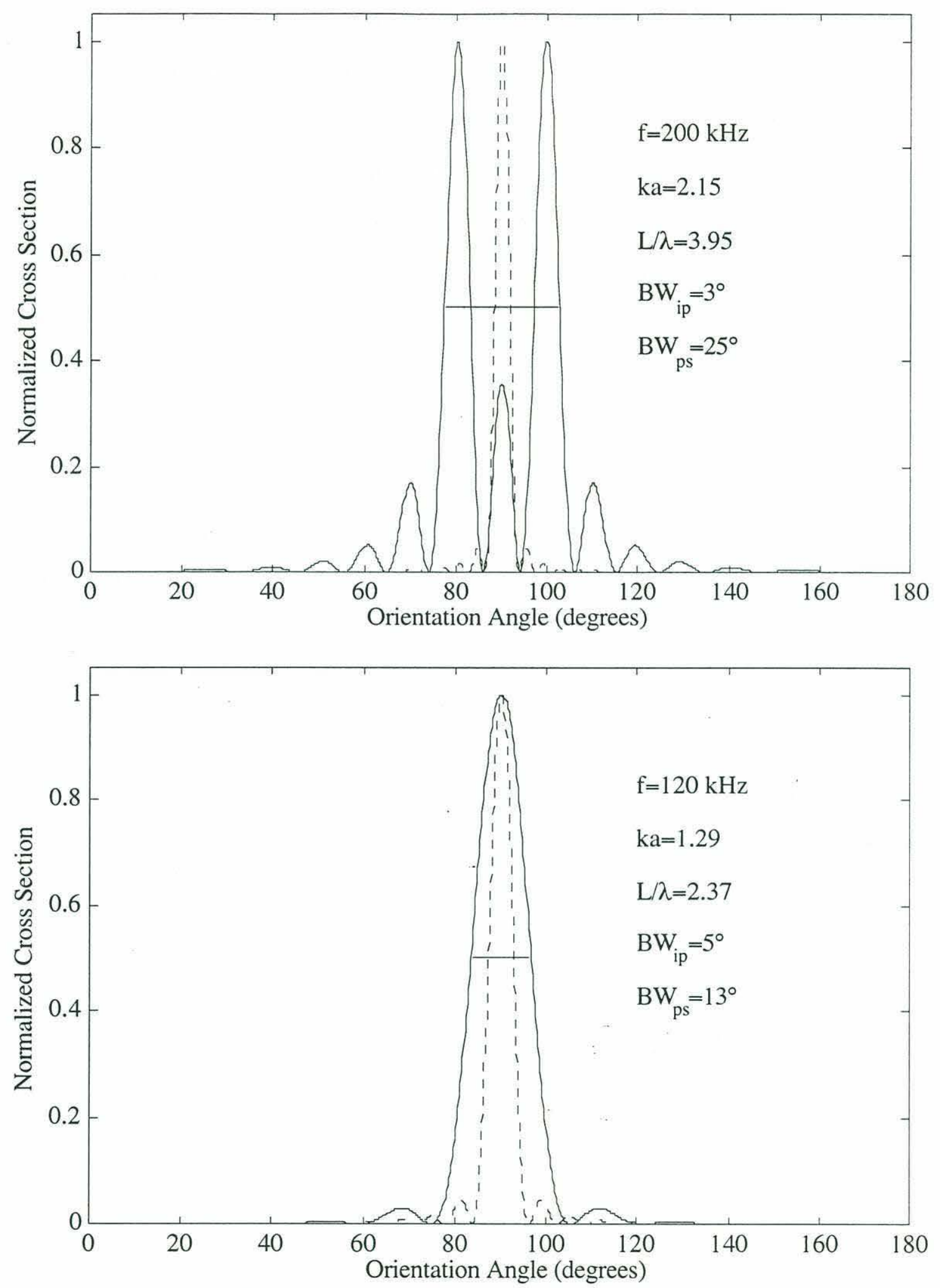

Figure 2-7: Comparison of backscatter directivity patterns for penetrable and impenetrable models [(-): backscatter directivity pattern for penetrable volume; (- -): backscatter directivity pattern for impenetrable surface.] 
Also plotted on Fig. 2-8 is the beamwidth as a function of acoustic frequency for the impenetrable surface. Comparing the penetrable and impenetrable cases, the impenetrable case shows a monotonic decrease in beamwidth with increasing acoustic frequency, while the penetrable case produces a non-monotonic function.

Figure 2-8 is plotted for a prolate spheroid $30 \mathrm{~mm}$ long and with a maximum radius of $2.6 \mathrm{~mm}$. These dimensions are based upon the average size of the animals used in the experiment. (Length was measured from the eyes to the tip of the telson.)

The effects on the plots of beamwidth versus $k a$ and $L / \lambda$ for the range of animal sizes used in the experiments are shown in Fig. 2-9. Varying the animal size had little effect on the plot of beamwidth versus $k a$. The predicted beamwidths at a given value of $k a$ varied no more than $2^{\circ}$ over the range of animal sizes used. The plot versus $L / \lambda$ shows a different effect. If the ratio $(L / 2 a)$ is constant, the predicted beamwidths for changing animal sizes match closely (within a couple of degrees). Changing the ratio $(L / 2 a)$ caused the plot to shift left and right - a decrease in the ratio caused a shift to the left. For the range of animal sizes and shapes used in the experiment, the maximum shift in the plot appeared to be approximately 10 percent of the value of $L / \lambda$. This is most noticeable in the regions where the step increases in beamwidth occur. 

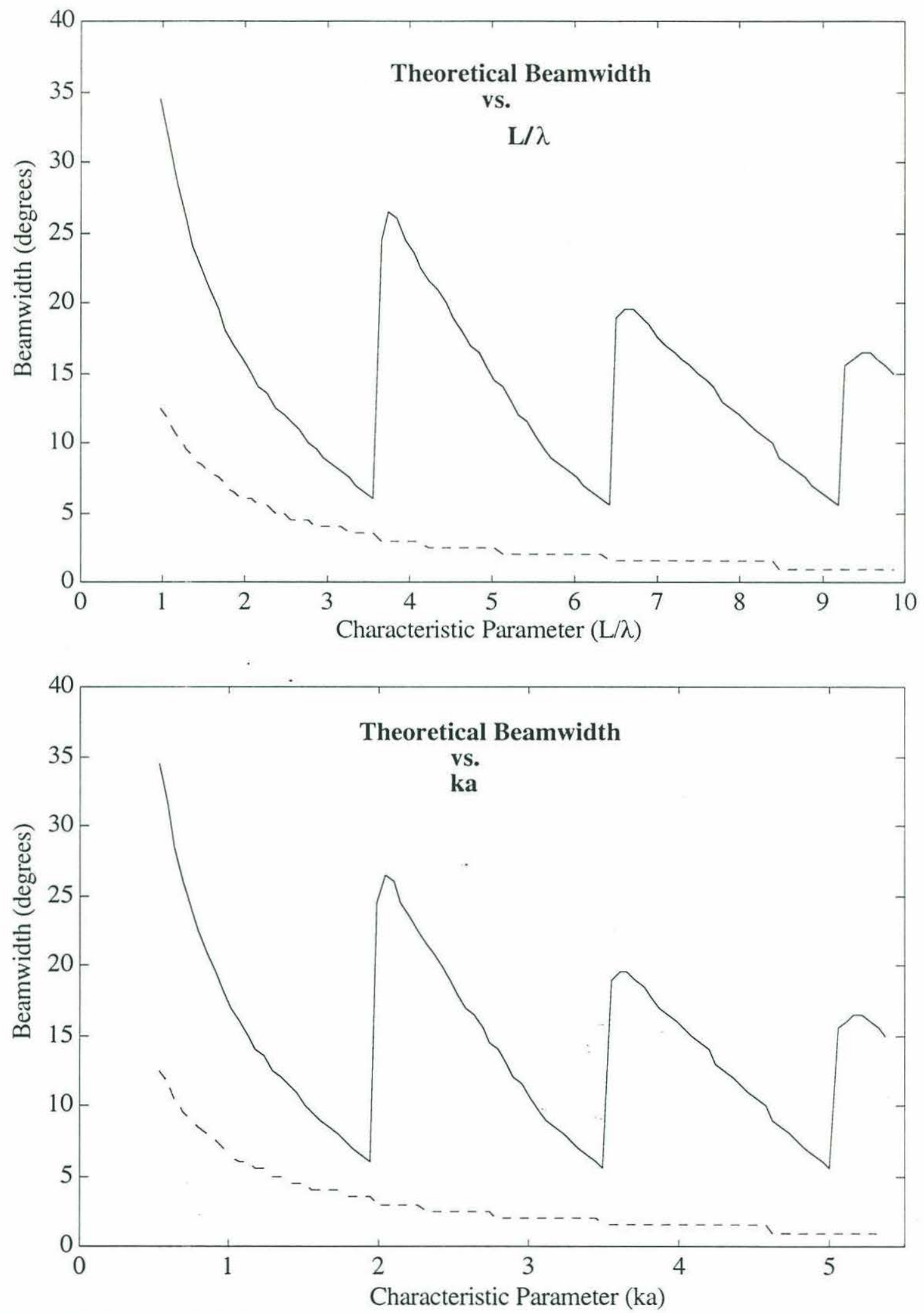

Figure 2-8: Beamwidth of the main lobe of the backscatter directivity pattern for a weak scattering prolate spheroid (-) and an impenetrable flat plate (- -). For this data, $L=30 \mathrm{~mm}$ and $a=2.6 \mathrm{~mm}$. 

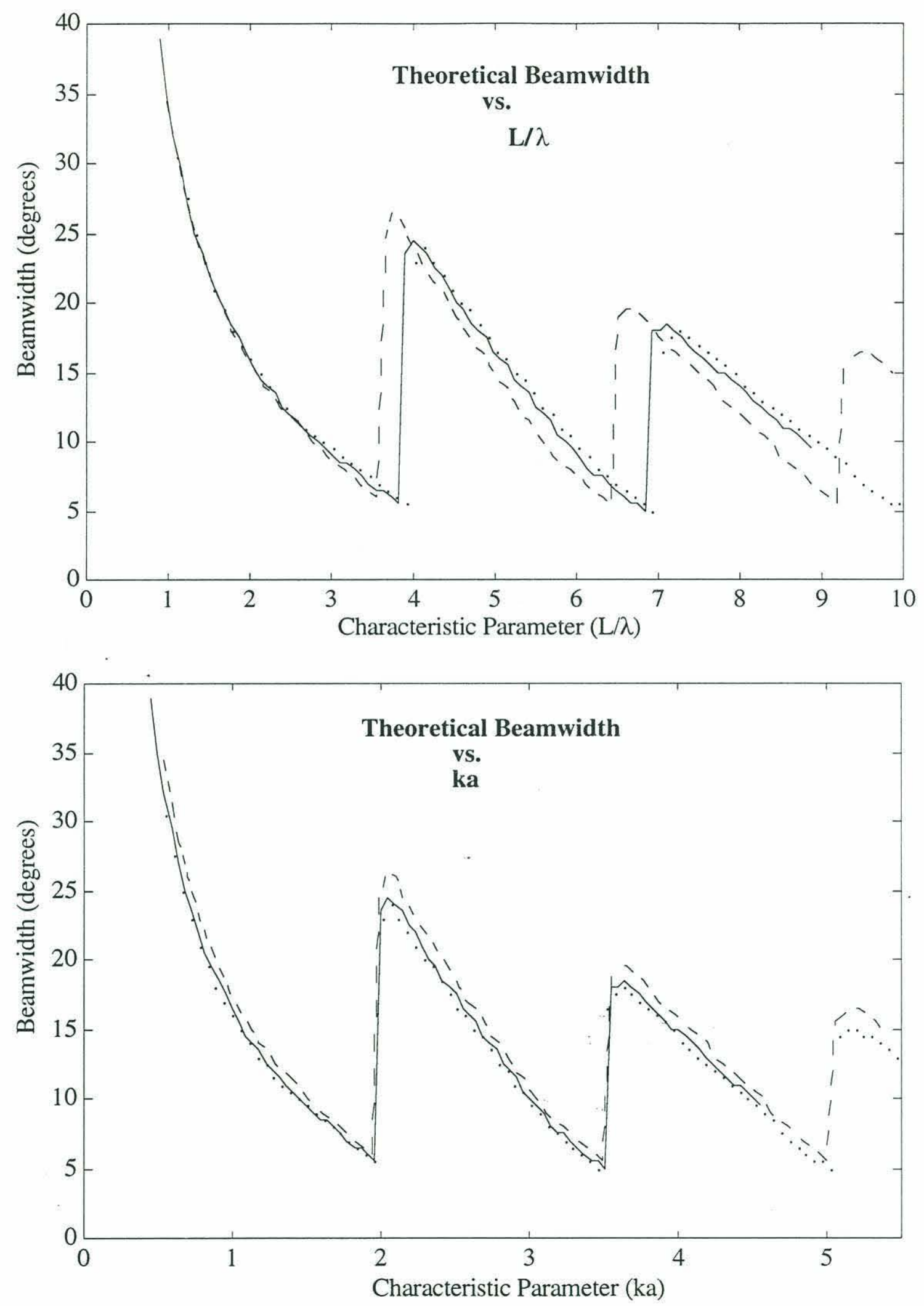

Figure 2-9: Effect of animal sizes on plots of beamwidth versus $k a$ and $L / \lambda$. [( -$): L=34 \mathrm{~mm}, a=2.7 \mathrm{~mm}$, $(L / 2 a)=6.3 ;(--): L=30 \mathrm{~mm}, a=2.6 \mathrm{~mm},(L / 2 a)=5.7 ;(\cdots): L=27 \mathrm{~mm}, a=2.2 \mathrm{~mm},(L / 2 a)=6.1]$ 


\subsection{Describing Simulated Data to Approximate Experimental Limitations}

The experiment restrained a live decapod shrimp with a snugly tied human hair in a seawater tank. Due to the imperfection of this restraint, the shrimp was able to move an estimated $\pm 3^{\circ}$ from the desired orientation angle, hence resulting in error in the scatter directivity measurements. It was assumed that the shrimp's deviation from the desired orientation angle could be modeled as a zero-mean Gaussian random variable with a variance of $3^{\circ}$. This deviation, $\Delta \beta$, was then added to the desired orientation angle $\left(\beta_{\text {desired }}\right)$ in the scattering amplitude equation [Eq. (2.111)]:

$$
\beta_{\text {actual }}=\beta_{\text {desired }}+\Delta \beta \text {. }
$$

For the experiment, the animal was insonified with typically five "pings" at each desired orientation angle. Figure 2-10 shows a plot of the angular deviation, averaged over five pings, versus orientation angle. The general characteristics of this figure agreed with observations of animal behavior made during the experiment.

The backscatter cross section of a prolate spheroid [Eq. (2.111)], is a continuous function of the orientation angle, but the measured data occurred at discrete angular increments of $3^{\circ}, 5^{\circ}$, or $10^{\circ}$. These discrete points were converted into a continuous function by means of a cubic spline algorithm. The combined effects of the animal motion within the restraint and the curve fitting algorithm would tend to smear and/or skew the microstructure of the backscatter directivity pattern. Figure 2-11 shows a comparison between the backscatter directivity pattern for a prolate spheroid and a "fitted curve" prolate spheroid with a $3^{\circ}$ Gaussian angular deviation. The beamwidth from a fitted curve $\left(\mathrm{BW}_{\mathrm{cf}}\right)$ could be either greater than or less than the beamwidth from an unmodified, perfectly restrained prolate 
spheroid $\left(\mathrm{BW}_{\mathrm{id}}\right)$. The difference is dependent upon the effect caused by the angular deviation $(\Delta \beta)$; while the process of curve fitting between discrete angular measurements tends to exaggerate the angular deviation effects.

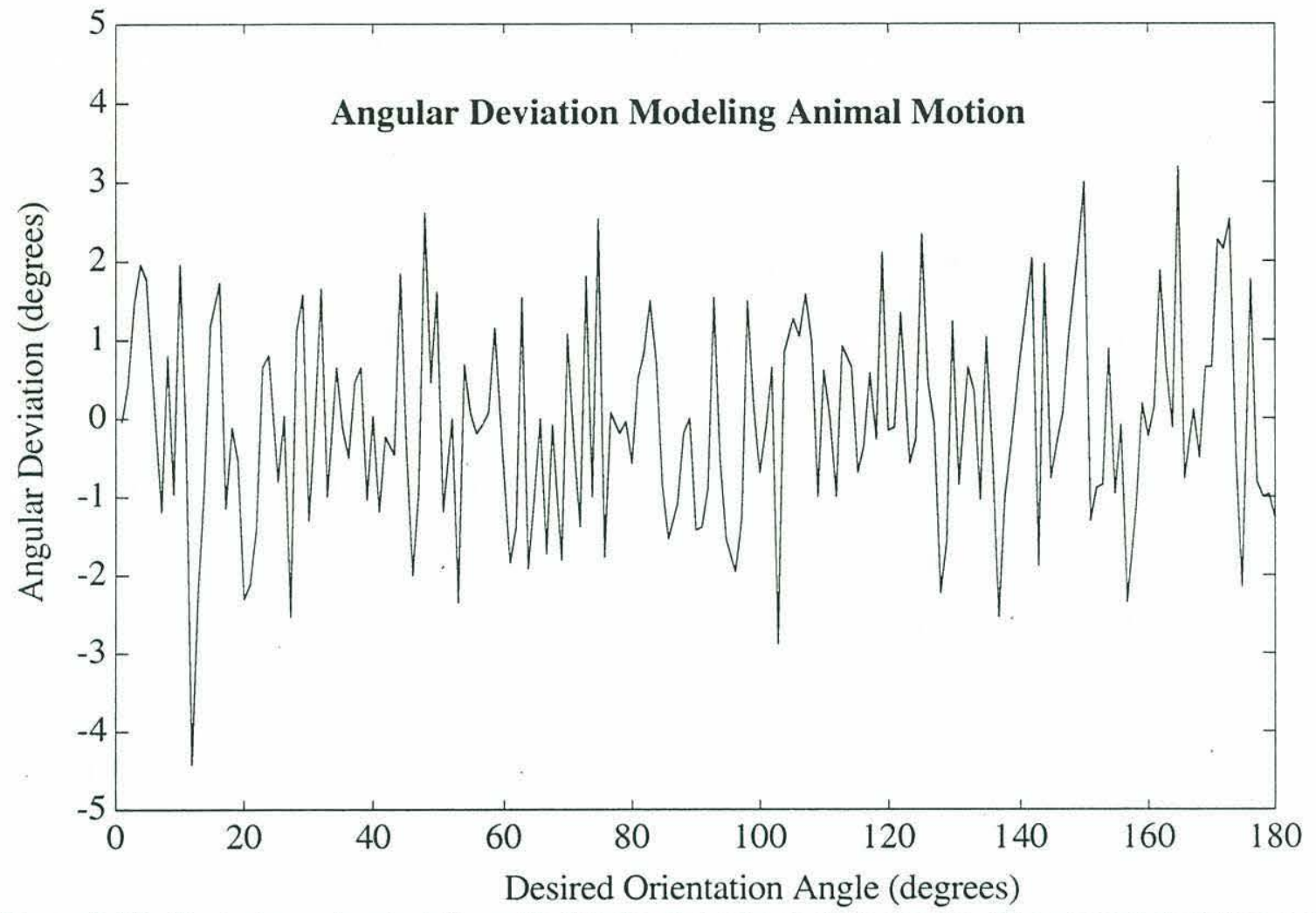

Figure 2-10: Typical angular deviation added to the desired orientation angle. The angular deviation is a zero-mean Gaussian random variable with a $3^{\circ}$ variance averaged over five pings. This factor was used to account for the movement of the decapod shrimp within its restraint.

The angular deviation is a Gaussian random variable, this allows the beamwidth as a function of frequency to be modeled as a Gaussian random process. Figure 2-12 depicts plots of the mean beamwidth as a function of $k a$ and $L / \lambda$. The plots were generated by calculating the beamwidth at a given frequency, taking into account the angular deviation and sampling at discrete angular positions separated by $5^{\circ}$. One hundred beamwidths were computed at each frequency. From this sample set, the mean and standard deviation for the beamwidths were determined. The mean theoretical data is plotted as a solid line $(-)$, the dashed lines (- -) represents the beamwidths that fall within one standard deviation of the 

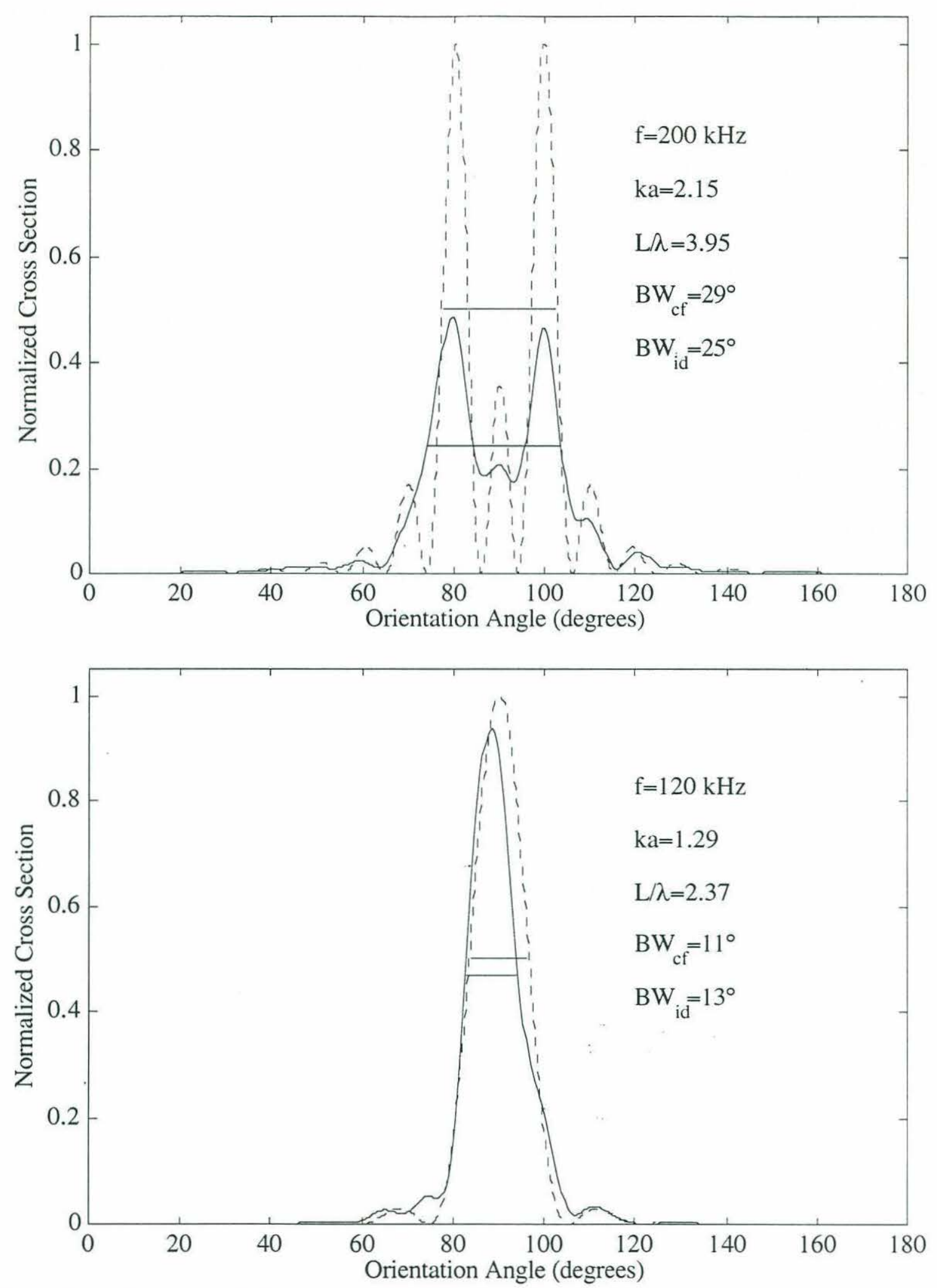

Figure 2-11: Comparison of the backscatter directivity pattern for an "ideal" non-moving prolate spheroid $(--)$ and the backscatter directivity pattern of the "curve fitted" prolate spheroid with animal motion within the restraint (一). ["Ideal" $\left(\mathrm{BW}_{\mathrm{id}}\right)$ and "curve fitted" $\left(\mathrm{BW}_{\mathrm{cf}}\right)$ beamwidths are shown as solid horizontal lines.] 
mean theoretical data, and the dotted lines $(\cdots)$ represent the minimum and maximum values of theoretical beamwidth predicted in the one hundred trial runs performed.

Comparing Fig. 2-12 to Fig. 2-8, it is noted that the mean beamwidth is greater than the beamwidth of the unmodified, perfectly restrained prolate spheroid over the range of frequencies evaluated. The unmodified beamwidth of Fig. 2-8 does fall within the maximum and minimum beamwidths found in the set of one hundred samples at each frequency. By modeling the theoretical data to reflect to limitations experienced during the collection of experimental data, the marked increases in the beamwidth at certain frequencies ( $k a$ and $L / \lambda$ ) were smoothed out. These step increases, at higher frequencies, were even more smoothed out. This can be seen by observing Fig. 2-12 at $L / \lambda$ between 3 and 4 , and $L / \lambda$ between 9 and 10 ; or $k a$ near 2 and $k a$ near 5.2. This phenomenon can be understood by referring to Fig. 2-6, and noticing that at higher frequencies, there are more and narrower significant side lobes. These narrow side lobes increase the effect of the angular deviation, a small change in angle is more likely to cause a large change in amplitude; this will cause larger fluctuations in the beamwidth, thus the smoothing of the step would be greater.

Comparing Fig. 2-12 to Fig. 2-9, over the range of animal sizes studied, the smearing due to animal motion and sampling limitations covers the changes in beamwidth versus $k a$ due to animal size differences. The shifts in the plots of beamwidth versus $L / \lambda$ due to changes in the ratio $(L / 2 a)$ are not completely hidden by the smearing from experimental limitations. This could add to the discrepancies between the experimental and theoretical results. 

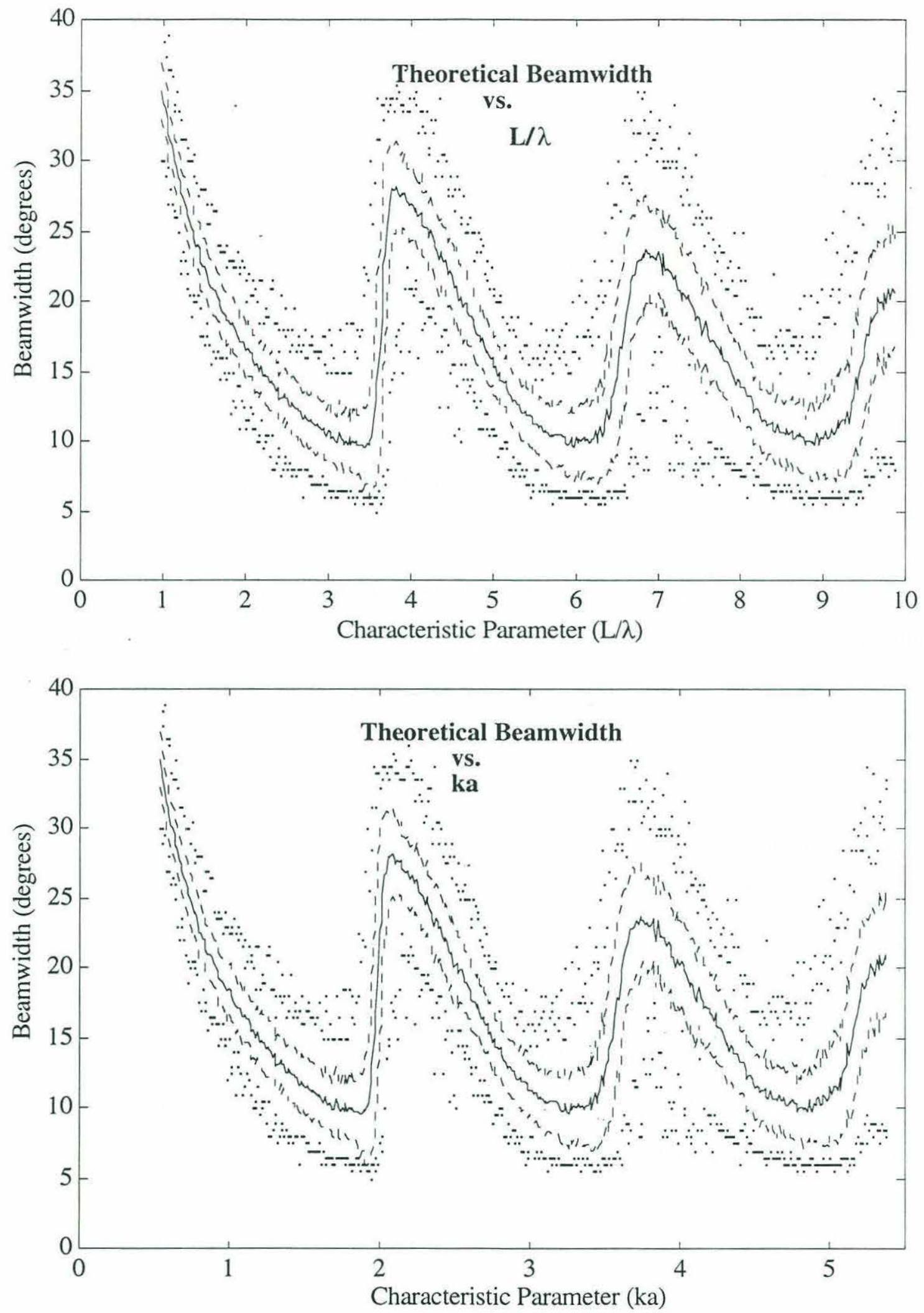

Figure 2-12: Mean beamwidth of the main lobe of the backscatter directivity pattern for a weak scattering prolate spheroid. (For this data, $L=30 \mathrm{~mm}$ and $a=2.6 \mathrm{~mm}$. Also depicted are the mean beamwidth plus/minus the standard deviation, and the maximum and minimum beamwidths computed.) 


\section{Chapter 3}

\section{Experimental Measurements}

\subsection{Overview of Apparatus and Procedures}

All of the experiments for this thesis were conducted in an acoustic pulse-echo laboratory at the Woods Hole Oceanographic Institute (Room G-8, Bigelow Laboratory). In these experiments, a live decapod shrimp was restrained in an acoustically transparent frame. The animal was then suspended in an 11,000 liter seawater tank $(360 \mathrm{~cm} \times 240$ $\mathrm{cm})$. It was manually rotated through $360^{\circ}$ in steps of $3^{\circ}, 5^{\circ}$ or $10^{\circ}$, while being insonified by a narrowband $(72,120,165,200,350,375,400,450,475$, and $525 \mathrm{kHz})$ or a broadband (320 to $487 \mathrm{kHz}$ chirp) acoustic transducer. The backscattered pressure wave was measured by a second transducer adjacent to the first, to resemble a monostatic geometry. For each transducer pair and angle of orientation, the backscattered pressure wave was amplified and sent through an appropriate bandpass filter: After repeating the measurement for all angles, the data set was stored and analyzed. The backscatter directivity pattern was determined, and the main lobe width of the directivity pattern was estimated. 


\subsection{Laboratory Arrangement}

\subsubsection{Mechanical Equipment and the Geometry of the Experiment}

There were three major pieces of mechanical equipment needed for the experiments: an acoustically transparent support to hold and restrain the animal, a method to support the transducers, and a seawater tank large enough to conduct the experiments.

The frame used to restrain the shrimp is depicted in Fig. 3-1. The animal is an extremely weak scatterer even at high frequencies; fine monofilament, thread, even horse hair proved to have a large enough backscatter cross section to adversely influence backscattering measurements of the animal. Human hair was found to be a sufficiently weak scatterer to allow measurements of the animal's backscattered pressure wave.

The side supports of the target frame (Fig. 3-2), although not acoustically transparent, could be eliminated from the data as the echoes arrived at times different than that of the animal (that is, the echoes from the supports were "gated out"). When the target was showing an end-on incidence with respect to the transducers, there was a time separation between the incident pressure wave reaching the target and either of the monofilament supports. The time separation, $\tau$, is equal to the displacement, $s$, divided by the speed of sound in water, $c$ :

$$
\tau=\frac{s}{c} .
$$




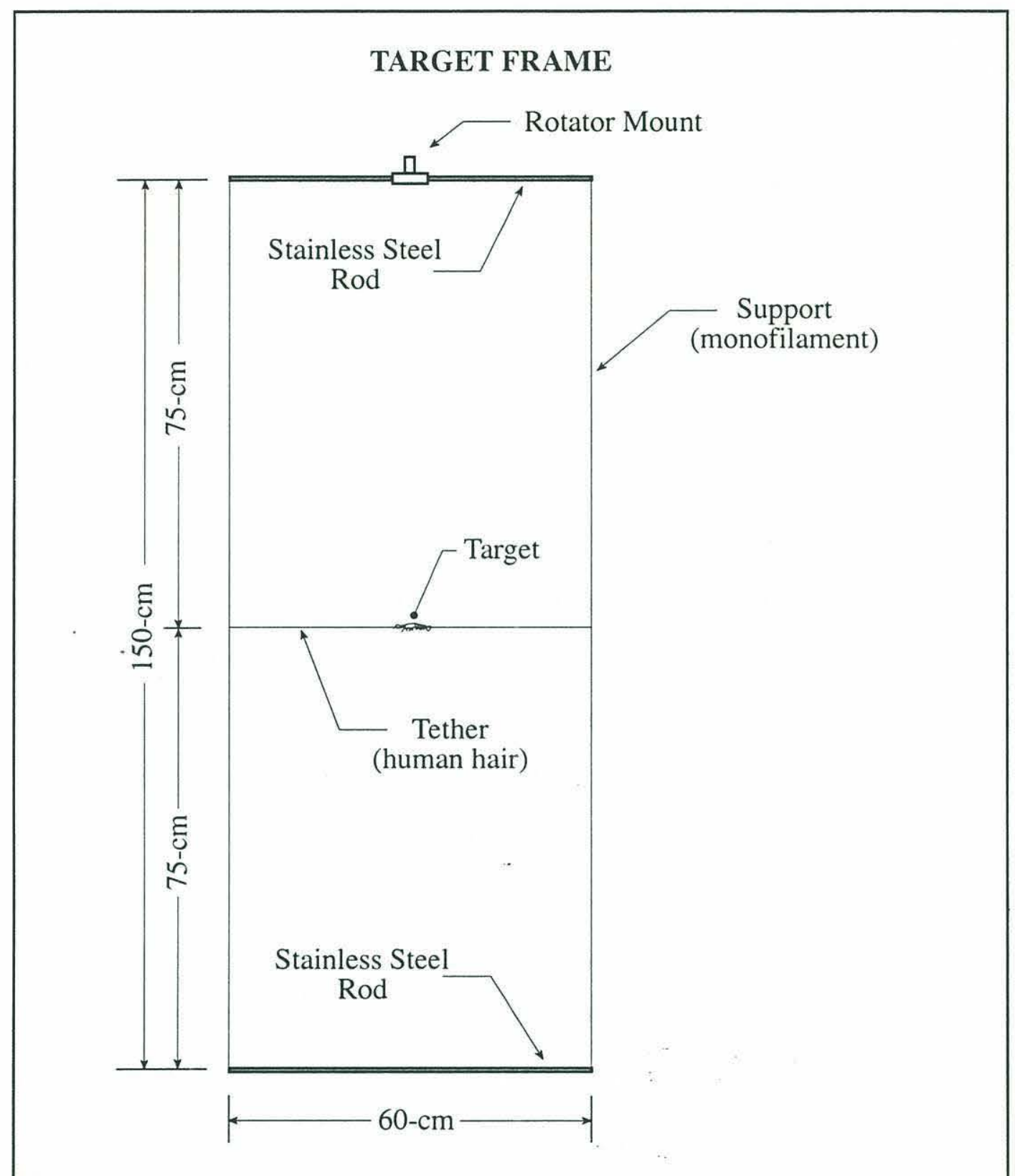

Figure 3-1: Target frame used to suspend a live animal in a seawater tank. [The top and bottom are made of stainless steel rods, 4-mm in diameter. The vertical supports consist of four-pound test fishing line (monofilament). The animal is tethered at the head and tail with a length of human hair.] 


\section{TARGET FRAME -TRANSDUCER GEOMETRY}

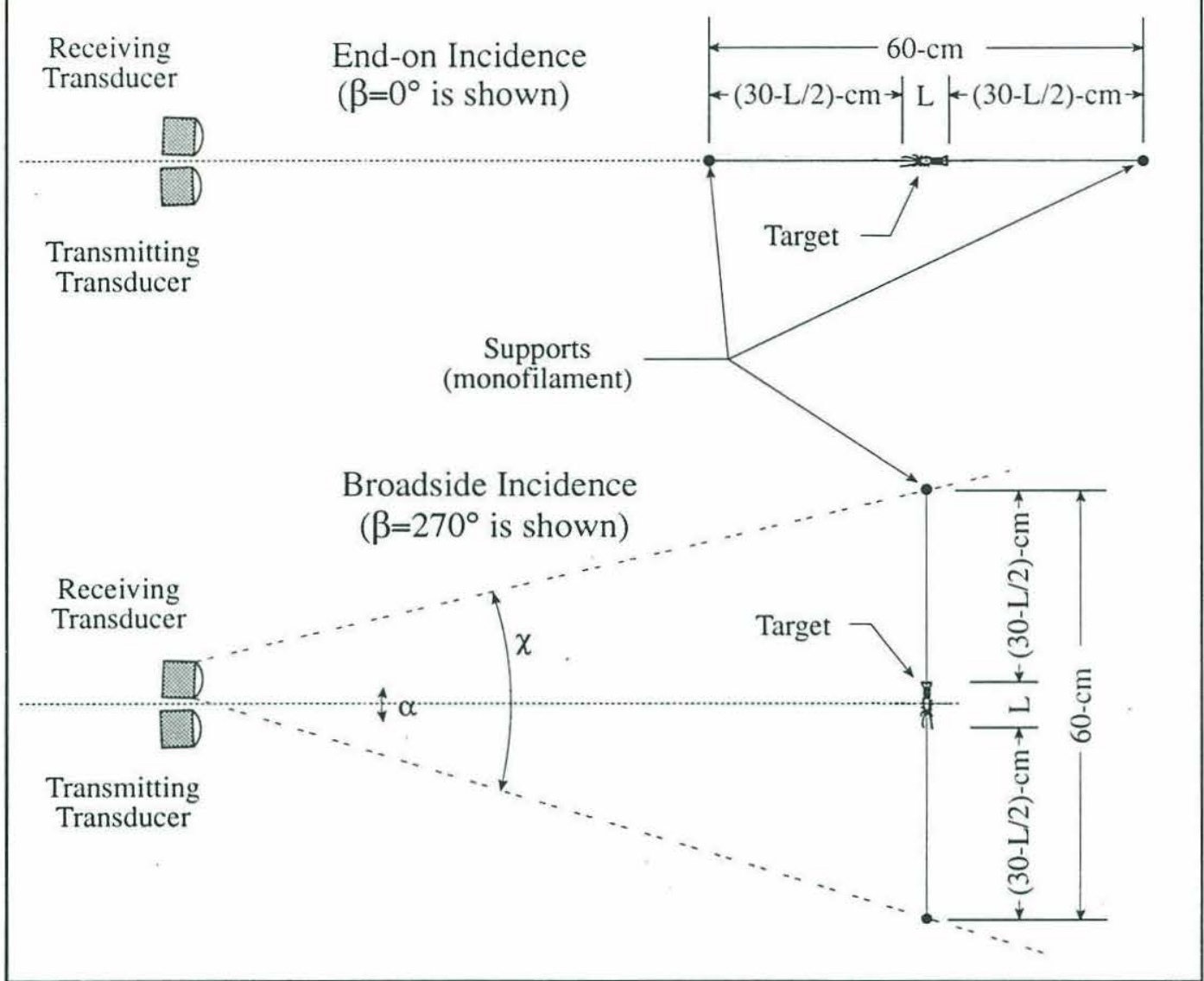

Figure 3-2: The target frame - transducer geometry designed to prevent the monofilament supports from interfering with the backscattered pressure signal from the shrimp (target). Top, geometry for end-on target orientation; bottom, geometry for broadside target orientation.

For these experiments, the maximum sound speed was $1525 \mathrm{~m} / \mathrm{s}$, and the largest animal had a length, $L$, of $3.4 \mathrm{~cm}$. Thus, the minimum time separation necessary is

$$
\begin{gathered}
\tau=\frac{\left(30-\frac{3.4}{2}\right) \mathrm{cm}}{1525 \frac{\mathrm{m}}{\mathrm{sec}}} \cdot \frac{1 \mathrm{~m}}{100 \mathrm{~cm}} \\
\tau=185.7 \mu \mathrm{sec} .
\end{gathered}
$$

If the incident pressure wave signal was less than $185.6 \mathrm{msec}$, at end-on incidence, the monofilament supports would not interfere with the scattering from the target. The longest input signal during these experiments was $100 \mathrm{msec}$. Referring to the geometries of Figs. $2-4$ and $3-2$, it is seen that

$$
s=d \cdot \cos (\beta)
$$


where

$$
d=\left(30-\frac{L}{2}\right) \mathrm{cm} .
$$

Rearranging Eq. (3.1), the maximum orientation angle, $\beta_{\max }$, for which a time separation would prevent the supports from interfering with the target is:

$$
\beta_{\text {max }}=\arccos \left[\frac{c \tau}{d}\right] .
$$

For the experiments:

$$
\begin{gathered}
\beta_{\max }=\arccos \left[\frac{0.1525 \frac{\mathrm{cm}}{\mu \mathrm{sec}} \cdot 100 \mu \mathrm{sec}}{\left(30-\frac{3.4}{2}\right) \mathrm{cm}}\right] \\
\beta_{\text {max }}=57^{\circ} .
\end{gathered}
$$

Conservatively assuming that the maximum orientation angle for which temporal separation ("time gating") of edges prevents support interference as $45^{\circ}$ (vice $57^{\circ}$ calculated above), the maximum allowable beamwidth for the receiving transducer, $\chi_{\max }$, is found to be (Fig. 3-2):

$$
\chi_{\text {max }}=2 \cdot\left\{\arctan \left[\frac{d \sin \left(\beta_{\max }\right)}{R+d \cos \left(\beta_{\max }\right)}\right]-\frac{\alpha}{2}\right\} .
$$

As before, $d$ is the distance separating the target from the support. The range from the transducer face to the target is $R$. The maximum value for the transducer angle, $\alpha$ was $6^{\circ}$. The maximum range was $110 \mathrm{~cm}$. This makes the maximum allowable beamwidth to prevent the supports from interfering with the backscattered pressure wave from the target:

$$
\begin{gathered}
\chi_{\max }=2 \cdot\left\{\arctan \left[\frac{\left(30-\frac{3.4}{2}\right) \mathrm{cm} \cdot \sin \left(45^{\circ}\right)}{110 \mathrm{~cm}+\left(30-\frac{3.4}{2}\right) \mathrm{cm} \cdot \cos \left(45^{\circ}\right)}\right]-\frac{6^{\circ}}{2}\right\} \\
\chi_{\text {max }}=11.5^{\circ} .
\end{gathered}
$$


The maximum beamwidth of the transducers used in this experiment was measured to be $8^{\circ}$, less than the maximum allowable value of $11.5^{\circ}$ (in this case "beamwidth" is the width of the main lobe of the diffraction pattern of the transducer). Thus, the target frame design was transparent. The frame was verified to be transparent by placing it within the seawater and rotating it through $360^{\circ}$ without a target in the frame. During these tests, the frame proved to be transparent.

The transducer frame (Fig. 3-3) was required to rigidly hold the transducer pairs in place within the seawater tank, yet allow focusing of, and rapid changing of the transducers. The frame also needed to provide for the transducer arrangement for bistatic calibration. The transducer frame produced met these requirements. Each transducer was held rigidly in a nylon block; the nylon blocks were each placed on their own stainless steel rod. The nylon blocks could be positioned vertically (z-direction) on the rod and locked in place. The transducer-block-rod composite could then be fitted in the combed top and bottom of the frame ( $y$-direction). The composite unit could be rotated in the $x-y$ plane and locked in place. The entire frame could be moved within the seawater tank and clamped into place ( $x$-direction).

The seawater tank was an 11,000 liter tank (Fig. 3-4). It was filled with filtered seawater from Woods Hole Harbor. The water had a lower salinity (31.6 ppt) than ocean seawater $(35 \mathrm{ppt})$. The mean temperature of the water ranged from $17^{\circ} \mathrm{C}$ to $23^{\circ} \mathrm{C}$. The corresponding sound speed can be calculated from [5]:

$$
c=1449.2+4.6 T-0.55 T^{2}+0.00029 T^{3}+(1.34-0.010 T)(S-35)+0.016 z,
$$

where $c$ is sound speed in meters per second, $T$ is temperature in degrees Celsius, $S$ is salinity in parts per thousand, and $z$ is depth in meters. Sound speeds for the temperatures and salinities measured during the experiments are tabulated in Table 3-1. 


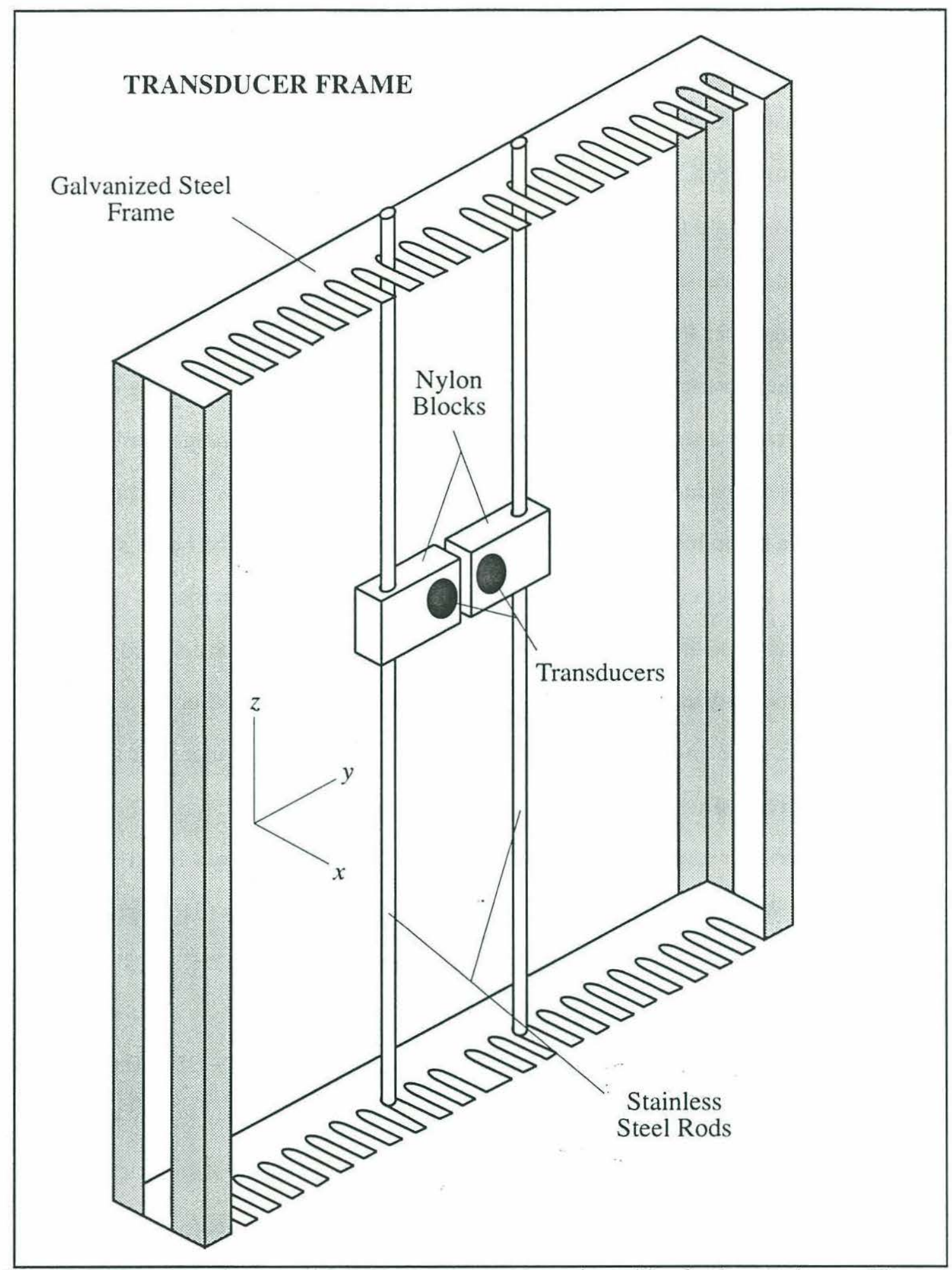

Figure 3-3: Transducer frame used to hold acoustic transducers in position for the experiment. (The transducers were held rigidly in custom made nylon blocks. The nylon blocks could be positioned vertically, and locked in place on $16 \mathrm{~mm}$ stainless steel rods. The rods could be positioned within the frame and rotated to the desired location, and then locked in place. This provided sufficient control of transducer position to perform the experiment.) 
Table 3-1.- Sound Speeds (m/s) for Various Temperatures and Salinities

\begin{tabular}{cccccccc} 
& \multicolumn{7}{c}{ Temperature $\left({ }^{\circ} \mathrm{C}\right)$} \\
Salinity (ppt) & 17 & 18 & 19 & 20 & 21 & 22 & 23 \\
31.5 & 1509 & 1512 & 1515 & 1518 & 1520 & 1523 & 1526 \\
31.6 & 1509 & 1512 & 1515 & 1518 & 1520 & 1523 & 1526 \\
31.7 & 1509 & 1512 & 1515 & 1518 & 1520 & 1523 & 1526
\end{tabular}

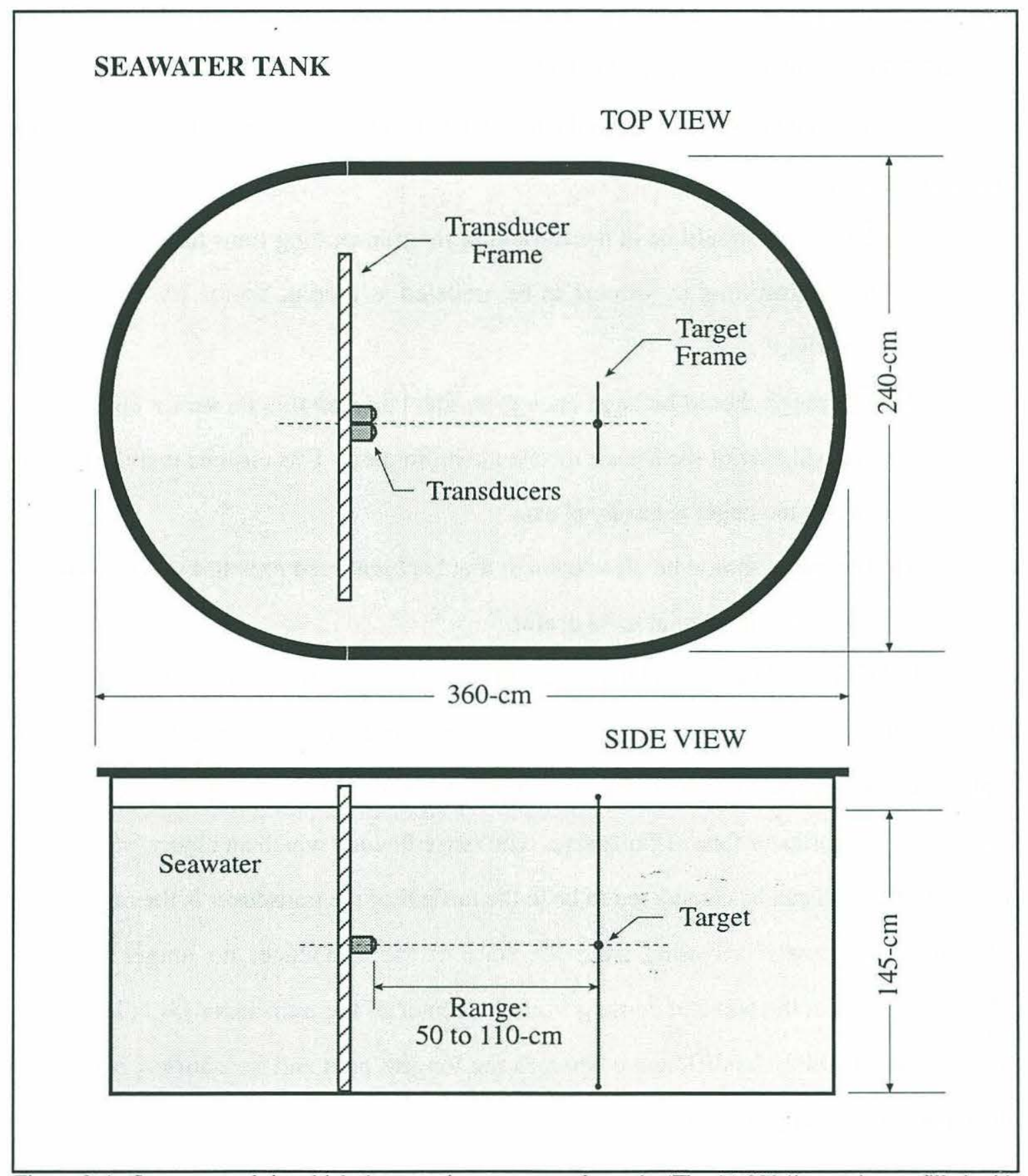

Figure 3-4: Seawater tank in which the experiment was performed. (The 11,000 liter tank was filled with filtered seawater.) 
If the tank was left for three or more days, a measurable temperature and salinity gradient was observed. This vertical gradient involved deviations of up to $1.1^{\circ} \mathrm{C}$ and $0.6 \mathrm{ppt}$, respectively. Since the medium (seawater) is assumed to be homogenous, this stratification was undesirable. By stirring the tank one or two days prior to any experiment, and then letting the tank settle for at least twelve hours, the seawater was found to be homogenous to within $0.05 \mathrm{ppt}$ and $0.1^{\circ} \mathrm{C}$.

The desired range, $r$ between the transducer faces and the target was determined by three criteria [5]:

1) The target should be in the farfield of the transmitting transducer. This allows the transmitting transducer to be modeled as a point source (with, of course, directivity).

2) The range should be large enough so that target should be well within the first Fresnel zone of the source/receiver combination. This ensures that the pressure field at the target is locally planar.

3) The range should be close enough that backscattered pressure wave produces a strong enough signal to be useful.

In the farfield of a transducer, the particle velocity is approximately in phase with the pressure. In this region the pressure wave is radiating outward with a uniform spherical divergence; thus the pressure is behaving as if it emanated from a point source rather than a transducer face of finite size. The range beyond which an object (at broadside to the transducer) can be considered to be in the farfield of the transducer is the range where the Huygens wavelet traveling from the edge of the transducer no longer interferes destructively with the wavelet coming from the center of the transducer [5]. (That is, the range beyond which the difference between the longest path and the shortest path is less than one-half an acoustic wavelength.) This produces the equation:

$$
R_{i n t}-R_{f f t} \lesssim \frac{\lambda}{2}
$$


where $R_{f f}$ is the range to the transducer's farfield from the center of the transducer face and $R_{\text {int }}$ is the range to the farfield from the edge of the transducer face. Applying the Pythagorean relation to the geometry of Fig. 3-5,

$$
R_{\text {int }}=\sqrt{R_{f f}^{2}+a_{\text {trans }}^{2}} \text {. }
$$

\section{FARFIELD CRITERIA}

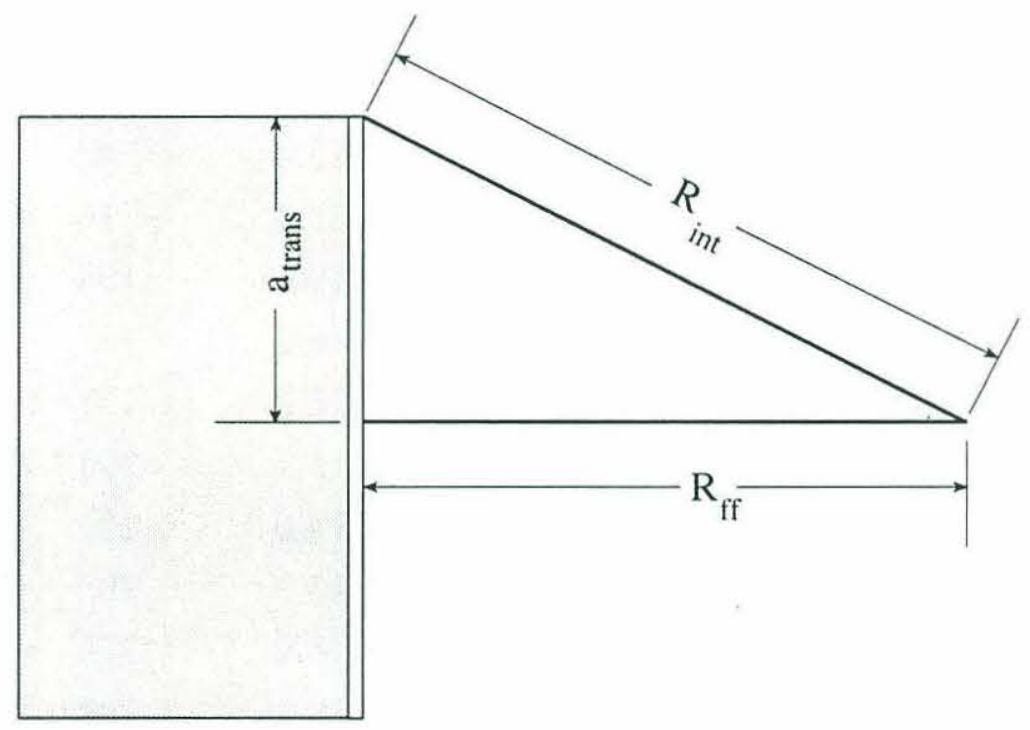

Transducer

Figure 3-5: Geometry for determining the range to the farfield of a piston transducer.

Substituting Eq. (3.7) into Eq. (3.6):

$$
\sqrt{R_{f f}^{2}+a_{t r a n s}^{2}}-R_{f f} \lesssim \frac{\lambda}{2}
$$

Solving for $R_{f f}$ :

$$
\begin{aligned}
\sqrt{R_{f f}^{2}+a_{\text {trans }}^{2}} & \lesssim \frac{\lambda}{2}+R_{f f} \\
R_{f f}^{2}+a_{\text {trans }}^{2} & \lesssim \frac{\lambda^{2}}{4}+\lambda R_{f f}+R_{f f}{ }^{2} \\
a_{\text {trans }}^{2} & \propto \frac{\lambda^{2}}{4}+\lambda R_{f f} \\
R_{f f} & \gtrsim \frac{a_{\text {trans }}^{2}}{\lambda}-\frac{\lambda}{4} .
\end{aligned}
$$


The American National Standards Institute has arbitrarily specified that [5]:

$$
R_{f f}=\frac{\pi a_{\text {trans }}^{2} .}{\lambda} .
$$

Comparing Eq. (3.9) to Eq. (3.10), it is obvious that Eq. (3.10) is a more restrictive requirement; it specifies a greater range to the transducer's farfield. The criterion of Eq. (3.10) was used to determine the minimum range. The ranges to the farfield for the transducers used are given in Table 3-2.

Table 3-2. - Distance to Farfield $\left(R_{f f}\right)$ for Various Transducers $(c=1520 \mathrm{~m} / \mathrm{s})$

$\begin{array}{rrrrr}f(\mathrm{kHz}) & \lambda(\mathrm{cm}) & a_{\text {trans }}(\mathrm{cm}) & R_{f f}(\mathrm{~cm}) & k R_{f f} \\ 72 & 2.11 & 3.0 & 13.4 & 40 \\ 120 & 1.27 & 3.0 & 22.3 & 111 \\ 165 & 0.92 & 2.4 & 19.6 & 134 \\ 200 & 0.76 & 2.0 & 16.5 & 137 \\ 350 & 0.43 & 1.3 & 12.2 & 177 \\ 375 & 0.41 & 1.3 & 13.1 & 203 \\ 400 & 0.38 & 1.3 & 14.0 & 231 \\ 450 & 0.34 & 1.3 & 15.7 & 292 \\ 475 & 0.32 & 1.3 & 16.6 & 326 \\ 525 & 0.29 & 1.3 & 18.3 & 398\end{array}$

The basic relation for the second range criterion is found by referring to Fig. 3-6, and noting the relationship:

$$
R-r=\Delta r .
$$

The first Fresnel zone is defined by the diameter, $D_{f}$, at which

$$
\Delta r=\frac{\lambda}{4} .
$$

Where $\Delta r=\lambda / 4$, the phase difference between the backscattered signal from the center of the Fresnel zone and the backscattered signal from the edge of the Fresnel zone is p. This is because the round trip (backscattered) distance traveled to the edge of the zone is one-half wavelength longer than the round trip distance to the center. The diameter of the first 
Fresnel zone $\left(D_{f}\right)$ is a function of target range $(r)$ and acoustic wavelength $(\lambda)$. From the Pythagorean relation:

$$
D_{f=} / 2=\sqrt{R^{2}-r^{2}} .
$$

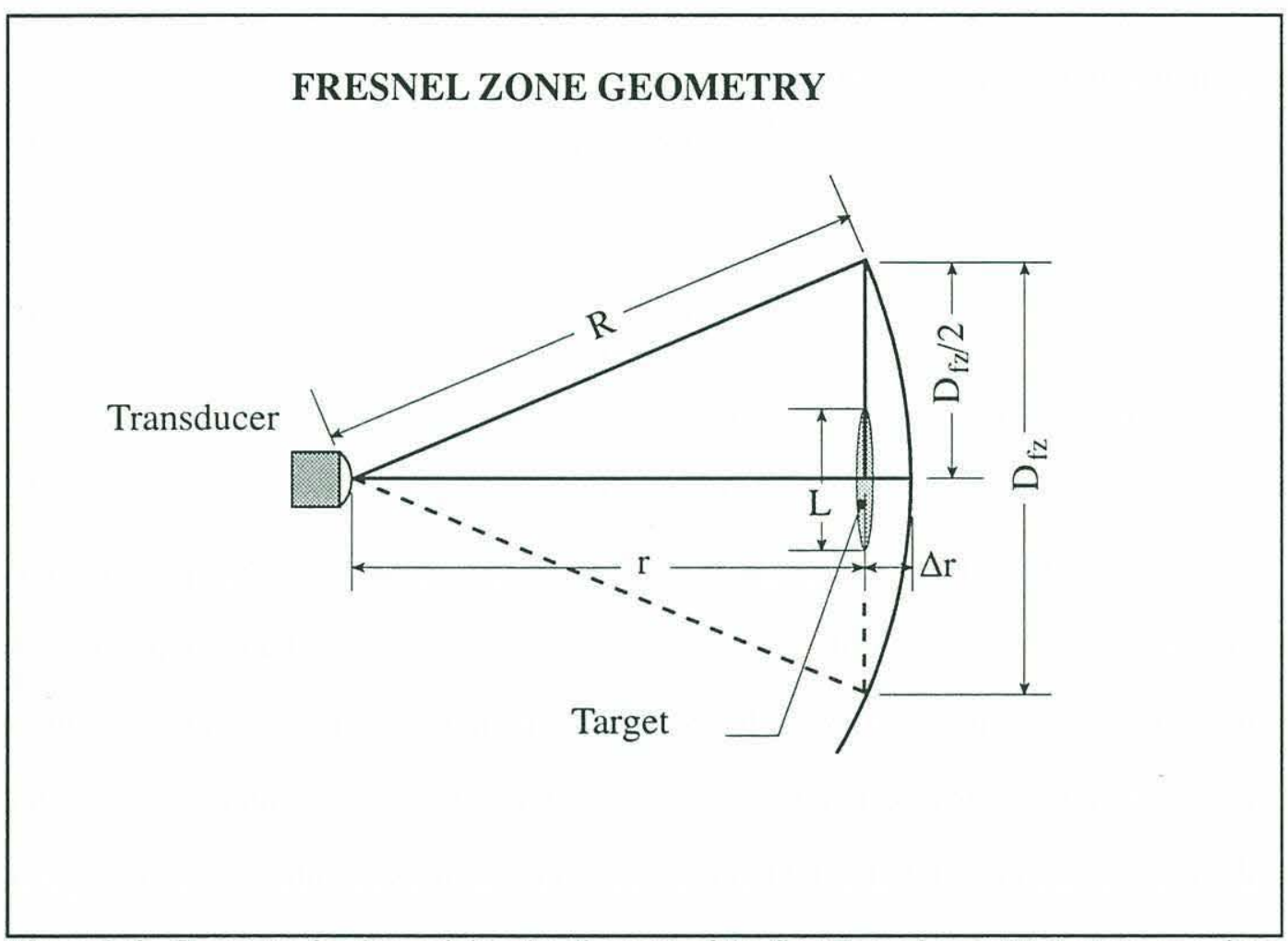

Figure 3-6: Geometry for determining the diameter of the first Fresnel zone $\left(D_{f}\right)$, at range $r$, from the transducer.

Rearranging and substituting Eqs. (3.11) and (3.12) into Eq. (3.13):

$$
\begin{aligned}
D_{f} / 2 & =\sqrt{(r+\Delta r)^{2}-r^{2}} \\
& =\sqrt{\left(r+\frac{\lambda}{4}\right)^{2}-r^{2}} \\
& =\sqrt{\left(r^{2}+\frac{r \lambda}{2}+\frac{\lambda^{2}}{16}\right)-r^{2}} \\
& =\sqrt{\frac{r \lambda}{2}+\frac{\lambda^{2}}{16}}
\end{aligned}
$$

or, 


$$
D_{f z}=\sqrt{2 \lambda\left(r+\frac{\lambda}{8}\right)} .
$$

The target range $(r)$ must be in the farfield of the transmitting transducer,

$$
r \geq R_{f f} .
$$

Applying this to the data in Table 3-2,

$$
\frac{2 \pi r}{\lambda}=k r>k R_{f f} \gg 1
$$

thus

$$
r \gg \frac{\lambda}{8}
$$

This allows Eq. (3.14) to be reduced to:

$$
D_{f z}=\sqrt{2 r \lambda} .
$$

Applying Eq. (3.18) to ranges of 50 and $100 \mathrm{~cm}$, the size of the first Fresnel zone is computed and tabulated in Table 3-3. These results show that at $100 \mathrm{~cm}$, the diameter of the first Fresnel zone is approximately four times the maximum length of the animal (3.4 $\mathrm{cm}$ ) for the three higher frequency transducers. The $72 \mathrm{kHz}$ transducer gave a very low signal-to-noise ratio (SNR) at a $100 \mathrm{~cm}$ range, and was moved into a $50 \mathrm{~cm}$ range to improve the signal strength. This produced a satisfactory SNR. From Table 3-3 it is clear that the target was well within the first Fresnel zone of the transducer.

Comparing Tables 3-2 and 3-3 to the transducer-to-target-range criteria listed above, it was found that a range of $50 \mathrm{~cm}$ was satisfactory for the $72 \mathrm{kHz}$ transducers and a range of $100 \mathrm{~cm}$ was satisfactory for the other transducers. Due to physical limitations within the seawater tank, the actual ranges used in the experiments were $54 \mathrm{~cm}$ and 107 $\mathrm{cm}$, respectively. These ranges were more conservative than those given in the tables, and thus the range criteria specified were still satisfied. 
Table 3-3.- Diameters of the First Fresnel Zone for Ranges of 50 and $100 \mathrm{~cm}$

$\begin{array}{rccc}f(\mathrm{kHz}) & \lambda(\mathrm{cm}) & \begin{array}{c}D_{f=}(\mathrm{cm}) \\ (r=50 \mathrm{~cm})\end{array} & \begin{array}{c}D_{f z}(\mathrm{~cm}) \\ (r=100 \mathrm{~cm})\end{array} \\ 72 & 2.11 & 14.5 & 20.5 \\ 120 & 1.27 & 11.3 & 15.9 \\ 165 & 0.92 & 9.6 & 13.6 \\ 200 & 0.76 & 8.7 & 12.3 \\ 350 & 0.43 & 6.6 & 9.3 \\ 375 & 0.41 & 6.4 & 9.1 \\ 400 & 0.38 & 6.2 & 8.7 \\ 450 & 0.34 & 5.8 & 8.2 \\ 475 & 0.32 & 5.7 & 8.0 \\ 525 & 0.29 & 5.4 & 7.6\end{array}$

\subsubsection{Equipment Set-up and Connections}

The data acquisition system (Fig. 3-7) was controlled by a personal computer. The specifics of the equipment used are given in Table 3-4.

All equipment was powered up at least thirty minutes prior to use to allow the devices to reach a steady operating temperature. Using a software package, $A T F$, developed by Bob Eastwood of WHOI, the computer would initialize the oscilloscope and prescribe the desired incident pressure wave equation into the function generator. The output from the function generator would then be sent to the transmitting transducer via the power amplifier. The function generator's output is also sent to the oscilloscope as a trigger for the oscilloscope. The data were collected bistatically, that is, there was a separate transmitting and receiving transducer, closely spaced to resemble a "monostatic" geometry. Cables to and from the transducers were rigged to give as much isolation as possible between the 
relatively high powered transmitted (incident) signal and the much lower powered received (backscattered) signal. This reduced the noise in the received signal.

The amplified signal excited the transmitting transducer causing it to emit an outgoing pressure wave. This incident pressure wave would strike the target and be scattered; the backscattered component was sensed by the receiving transducer. The receiving transducer converted the backscattered pressure wave to an electrical signal. This low powered electrical signal was amplified and sent to the bandpass filter. The bandpass filter rejected frequencies above and below the transducer frequency, thus improving the signal-to-noise ratio of the received electrical signal. The settings on the bandpass filtered are tabulated in Table 3-5. The settings were determined empirically, during the calibration phase of the experimental procedure, by adjusting the bandpass filter while observing the received calibration signal. The bandpass filter setting was adjusted by decreasing the width of the pass band around the central transducer frequency until signal reduction was observed. The pass band was then increased approximately ten-percent to ensure that there would be no reduction of the signal by the bandpass filter.

The modified received signal was then sent to the digital oscilloscope. The signal was further refined within the oscilloscope by subtracting off the systematic noise within the seawater tank. During the alignment phase of the experimental procedure, approximately one hundred pulses were averaged without the target in the seawater tank. The random noise would average out to nearly zero, while the systematic noise would remain. The systematic noise was due to reflections from surfaces within the tank such as the sides and bottom of the tank, and the surface of the water. This averaged noise was stored in the oscilloscope and subtracted from the signals received from the target in real time. The refined signal was stored in the computer for future analysis. 
Table 3-4.-Electronic Equipment used in the Data Acquisition System

$\begin{array}{ll}\text { Equipment: } & \text { Description: } \\ \text { Computer } & \begin{array}{l}\text { DTK Model FEAT-5030, 486DX-50MHz } \\ \text { Computer }\end{array} \\ \text { Oscilloscope } & \begin{array}{l}\text { LeCroy 9430, 10-bit 100-Msample/sec Digital } \\ \text { Oscilloscope }\end{array} \\ \text { Function Generator } & \begin{array}{l}\text { Analogic, Data Precision Polynomial Waveform } \\ \text { Synthesizer, Model 2020 }\end{array} \\ \text { Bandpass Filter } & \begin{array}{l}\text { A.P. Circuit Corporation, Variable Frequency } \\ \text { Filter }\end{array} \\ \text { Power Amplifier } & \text { ENI Model 2100L RF Power Amplifier } \\ \text { Pre-amplifier } & \text { Constructed at WHOI } \\ \text { Power Supply } & \text { Global Specialties Model 1301A Power Supply } \\ \text { Transmit/Receive Switch } & \text { Constructed at WHOI }\end{array}$

Table 3-5.- Bandpass Filter Settings

Transducer Frequency Bandpass Filter Settings

$\begin{array}{rr}72 \mathrm{kHz} & 24-160 \mathrm{kHz} \\ 120 \mathrm{kHz} & 60-200 \mathrm{kHz} \\ 165 \mathrm{kHz} & 60-400 \mathrm{kHz} \\ 200 \mathrm{kHz} & 60-500 \mathrm{kHz} \\ 350 \mathrm{kHz} & 200-800 \mathrm{kHz} \\ 375 \mathrm{kHz} & 200-800 \mathrm{kHz} \\ 400 \mathrm{kHz} & 200-800 \mathrm{kHz} \\ 450 \mathrm{kHz} & 200-900 \mathrm{kHz} \\ 475 \mathrm{kHz} & 200-1000 \mathrm{kHz} \\ 525 \mathrm{kHz} & 200-1000 \mathrm{kHz} \\ .25-.55 \mathrm{MHz} \text { chirp } & 100-1000 \mathrm{kHz}\end{array}$




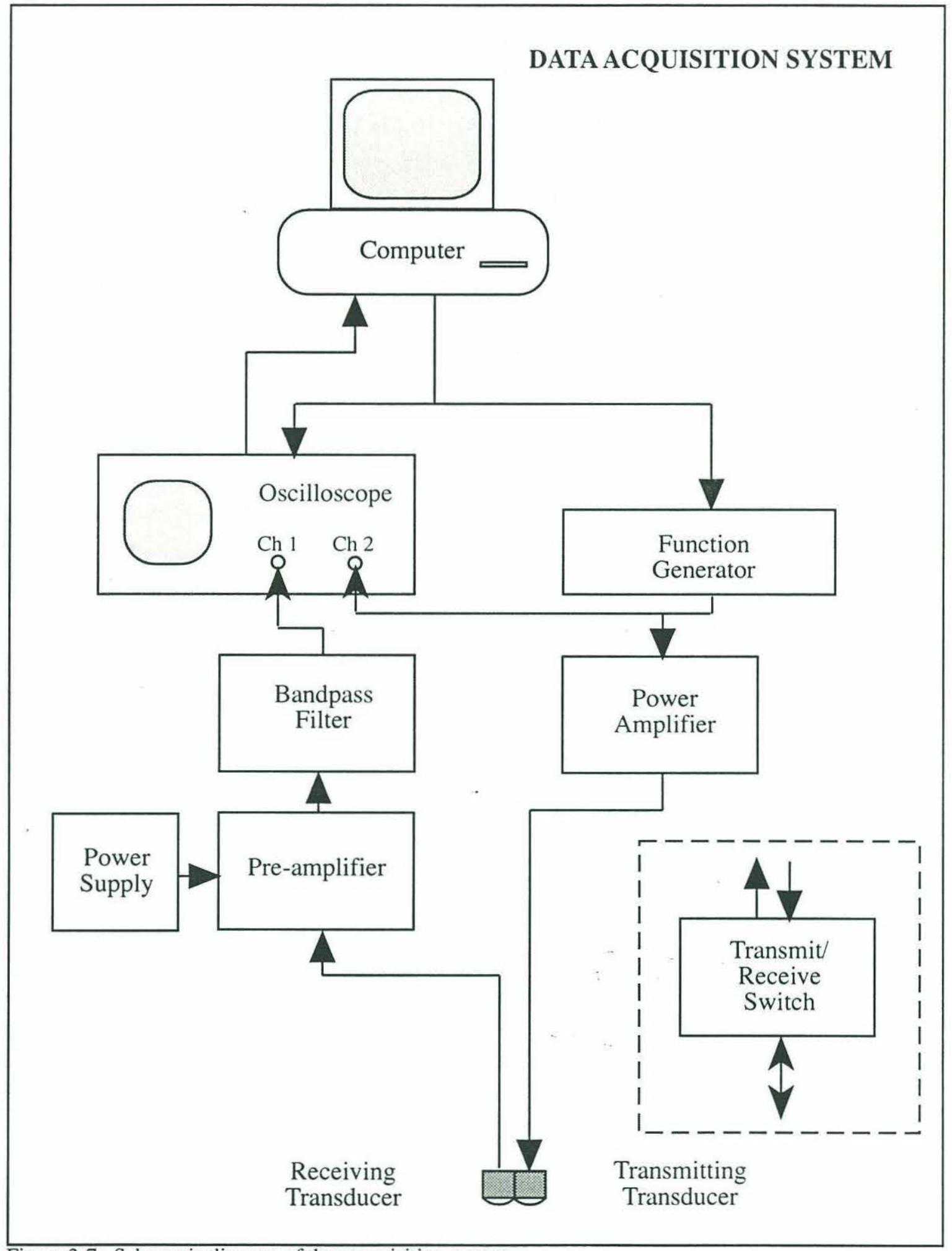

Figure 3-7: Schematic diagram of data acquisition system. 


\subsection{Experimental Procedure}

Once the laboratory had been set up as described in the previous section, the experiment was performed using a given pair of transducers. The pair was carefully placed in the transducer frame. After extended submergence, nucleate bubbles would form on the transducer faces. The presence of these bubbles could corrupt the data. To prevent the formation of these bubbles, the transducer faces were coated with a liquid detergent while remaining in the water. Similar bubbles were also observed to form on the decapod shrimp and the human hair tether. These were removed by carefully tapping the target frame to break the bubbles free from the animal, and also carefully wiping fingers along the human hair to free bubbles from the tether.

All actions in and around the tank were performed with slow deliberation to prevent disturbing the seawater. The higher frequency gravity waves in the seawater tank would dissipate within minutes, but lower frequency gravity waves appeared to cause fluctuations in the backscattered wave several hours later. This was especially evident at lower acoustic frequencies with a temperature and/or salinity gradient in the seawater tank. 


\subsubsection{System Calibration}

The first phase of the experiment was calibration of the system. The purpose of the system calibration is to accurately measure the overall system sensitivity. The system sensitivity produced a reference to which the data collected could be compared to. It also provided a quick check that the equipment was operating correctly.

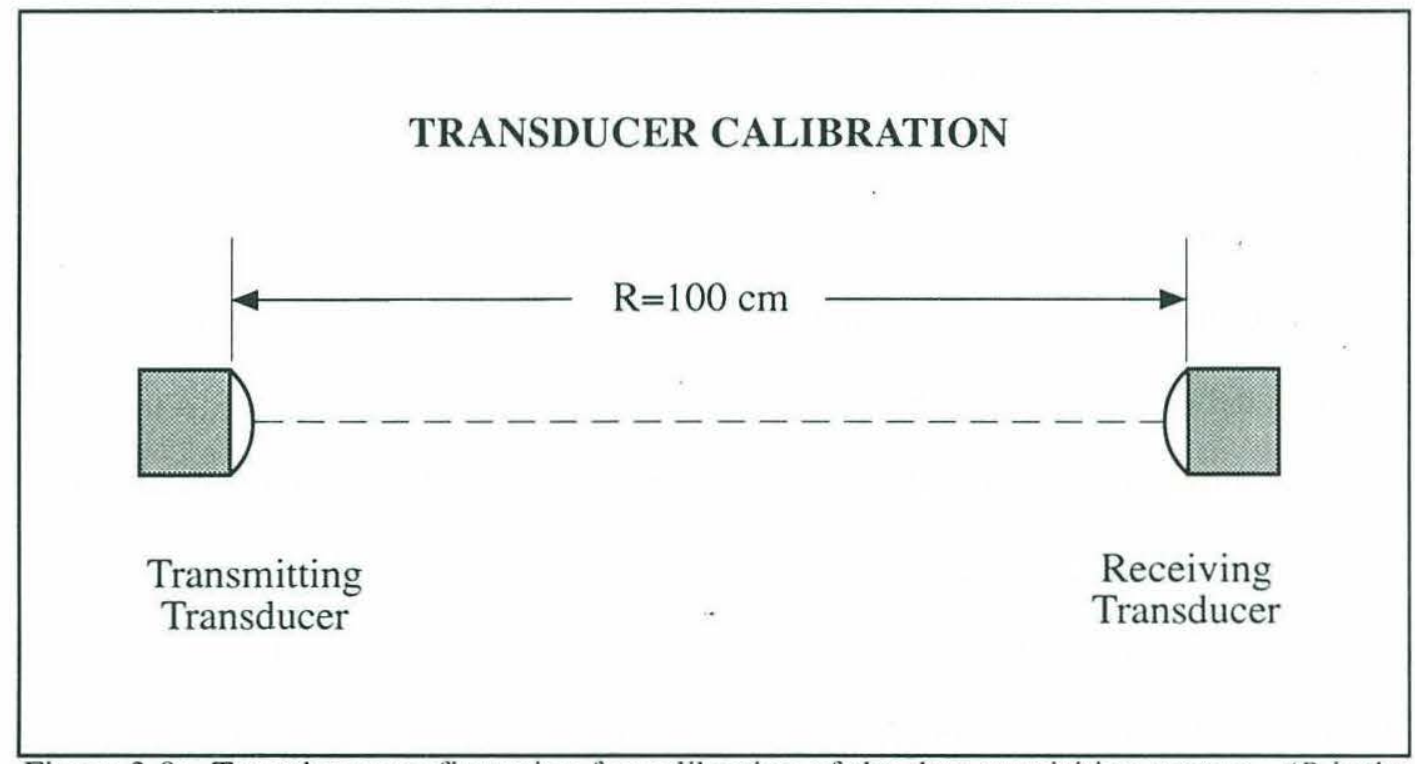

Figure 3-8: Transducer configuration for calibration of the data acquisition system. $(R$ is the range between transducer faces. During the actual measurements, the range was $98 \mathrm{~cm}$, vice $100 \mathrm{~cm}$, due to physical limitations within the transducer frame.)

The calibration was performed by having the transducers face each other in the transducer frame, approximately $100 \mathrm{~cm}$ apart (Fig. 3-8). While observing the received signal on the oscilloscope, the transducers were individually rotated about $10^{\circ}$ in each direction, noting the angular position at which the observed signal was at a maximum. Both transducers were then placed at the angular positions which produced the greatest signal. 
The input and maximum output voltages were recorded, and the maximized received signal was stored in the computer's memory.

\subsubsection{Alignment of Transducers and Measurement of Systematic Noise}

TRANSDUCER ALIGNMENT

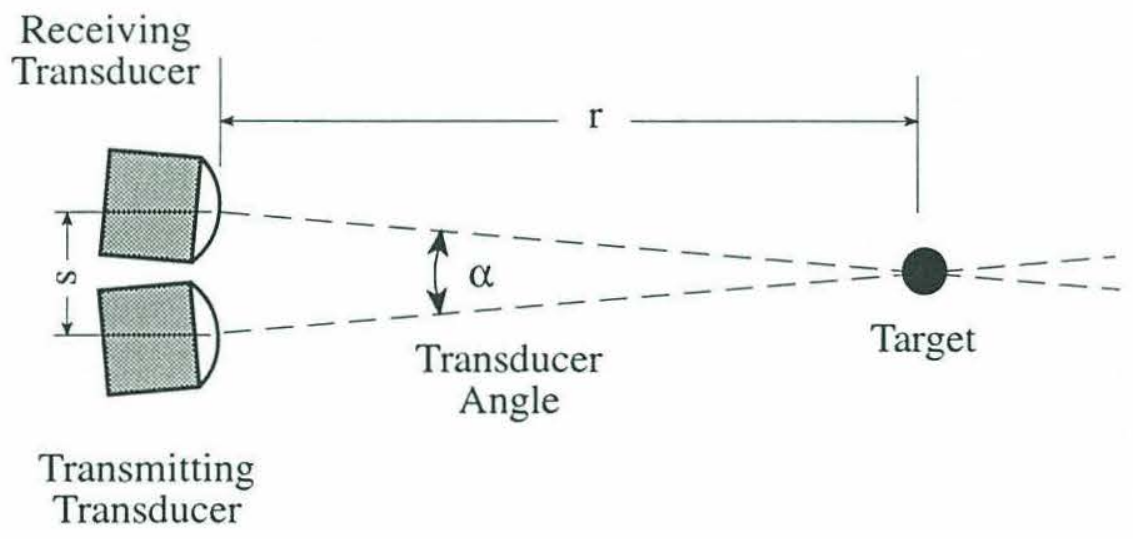

Figure 3-9: Configuration for transducer alignment. ( $r$ is the range from the transducer face to the target; $s$ is the centerline separation between the transducers; a is defined as the "transducer angle".)

After the system calibration, the transducers had to be aligned such that the center of the beam of each transducer was aimed at the location where the target would be. This was done by connecting each transducer, in turn, in a monostatic configuration. Figure 3-7 shows the bistatic configuration in which there are two transducers: one transmitting and one receiving. For the monostatic configuration, only one transducer is used, the transmit/receive switch was connected into the system with only one transducer in use. 
The transmit/receive switch would cause the single transducer to act alternately as a transmitter and a receiver.

The centerlines of the transducers were physically separated, thus there was some transducer angle, a, at which the transducers would be focused on the target (Fig. 3-9). Table 3-6 gives the transducer angle for the transducers used at ranges of 54 and $107 \mathrm{~cm}$. The formula for computing the transducer angle is:

$$
\alpha=\arctan \left[\frac{s / 2}{r}\right]
$$

where $\alpha$ is the transducer angle, $s$ is the distance between the centerlines of the transducer faces, and $r$ is the range between the target and the transducer.

Table 3-6.- Transducer Angles at Ranges of 54 and 107 centimeters

$\begin{array}{rccc}\text { Transducer } & \begin{array}{c}\text { Centerline-to-Centerline } \\ \text { Frequency }\end{array} & \begin{array}{c}\text { Transducer Angle } \\ (r=54 \mathrm{~cm})\end{array} & \begin{array}{c}\text { Transducer Angle } \\ (r=107 \mathrm{~cm})\end{array} \\ 72 \mathrm{kHz} & 10.8 \mathrm{~cm} & 5.7^{\circ} & 2.8^{\circ} \\ 120 \mathrm{kHz} & 7.4 \mathrm{~cm} & 3.9^{\circ} & 2.0^{\circ} \\ 165 \mathrm{kHz} & 5.2 \mathrm{~cm} & 2.8^{\circ} & 1.4^{\circ} \\ 200 \mathrm{kHz} & 5.2 \mathrm{~cm} & 2.8^{\circ} & 1.4^{\circ} \\ 500 \mathrm{kHz} & 4.6 \mathrm{~cm} & 2.4^{\circ} & 1.2^{\circ}\end{array}$

The alignment was performed by placing a 25 -mm-diameter steel ball (suspended by monofilament) at the same position in the seawater tank that the decapod shrimp would occupy. Then, with the "receiving" transducer connected in a monostatic configuration, it was rotated $\alpha / 2$ degrees to the right of the $x$-axis (see Fig. 3-3). From this position, it was rotated $10^{\circ}$ in each direction. During the rotation, the received signal was monitored on the oscilloscope, the angular position where the received signal was at a maximum was noted. The "receiving" transducer was placed at the noted angular position and locked in place. This procedure was then repeated for the "transmitting" transducer. 
After aligning both transducers monostatically, they were restored to the bistatic configuration. The steel ball was then moved up and down, from side to side, and toward and away from the transducers. While the ball was being moved, the received signal was observed on the oscilloscope. This was to verify that, in the bistatic configuration, the system was focused on the target, and that the beams were narrow enough that scattering from the frame would not interfere with scattering from the target.

The steel ball was removed, and the systematic background noise was measured by averaging at least 100 scattered signals with the target not in the seawater tank. This background signal was stored in the oscilloscope's memory and used to refine the data collected, in real time, from the backscattering off of the decapod shrimp.

\subsubsection{Data Acquisition}

The data was attained by carefully placing the target frame and target in the seawater tank, at the focal point of the transducers. The animal was then visually aligned with its head toward the transducers. This "end-on" incidence was defined as $\beta=0^{\circ}$. The animal was then insonified one, ten, fifteen, or twenty times and rotated three, five, or ten degrees, depending on the data set. In each data set the animal was rotated a total of $360^{\circ}$ in either the clockwise or counter-clockwise direction. The data sets are summarized in Table 3-7.

The number of insonification events (pings) and the angular increments were varied to provide a sufficient number of data points, at a sufficient number angular positions to provide an accurate mapping of the backscatter directivity pattern of the animal. Additionally, the data had to be collected rapidly enough so that the animal's motion would be consistent (active swimming or passive resting) throughout the data taking process. 
With the apparatus available, both of these criteria could not be met. Thus, on data runs 4 through 7 both "fast angular sweep" and "slow angular sweep" data sets were acquired. A fast angular sweep data set consisted of performing one insonification event every $3^{\circ}$ for $360^{\circ}$. This took approximately 90 seconds to perform, but the data was more irregular than slow angular sweep data sets. The slow angular sweep data sets generally consisted of fifteen insonification events every $5^{\circ}$ for $360^{\circ}$. These data sets took approximately 75 minutes. The slow angular sweep data sets tended to smear the structure of the backscatter directivity pattern, but gave more consistent results.

The rotation direction was varied in data runs 5 through 15 because of the asymmetries between port and starboard beamwidths measured. By comparing the port and starboard beamwidths in both the clockwise and counter-clockwise directions it was determined that the asymmetries were due to the stochastic nature of the measurements and animal movement, instead of being due to physical differences.

For data runs 9 through 15 a broadband $500 \mathrm{kHz}$ transducer was used. At these higher frequencies (from 350 to $525 \mathrm{kHz}$ ), the fast angular sweep data sets produced no useful data due to lower SNR and large irregularities at the higher acoustic frequencies. 
Table 3-7.- Animal Size and Data Sampled in the Experiments

\begin{tabular}{|c|c|c|c|c|c|c|}
\hline $\begin{array}{l}\text { Data Set } \\
\text { Number }\end{array}$ & Animal & $\begin{array}{l}\text { Length } \\
\text { (L) }\end{array}$ & $\begin{array}{c}\text { Mean } \\
\text { Radius (a) }\end{array}$ & $\begin{array}{l}\text { Pings per } \\
\text { Angular } \\
\text { Step }\end{array}$ & $\begin{array}{l}\text { Size of } \\
\text { Angular } \\
\text { Step }\end{array}$ & $\begin{array}{l}\text { Direction } \\
\text { of } \\
\text { Rotation }\end{array}$ \\
\hline 1 & $\# 1$ & $30.2 \mathrm{~mm}$ & $2.5 \mathrm{~mm}$ & 20 & 10 & $\mathrm{CW}$ \\
\hline 2 & $\# 2$ & $27.1 \mathrm{~mm}$ & $2.4 \mathrm{~mm}$ & 10 & 5 & $\mathrm{CW}$ \\
\hline 3 & $\# 3$ & $26.2 \mathrm{~mm}$ & $2.0 \mathrm{~mm}$ & 15 & 5 & $\mathrm{CW}$ \\
\hline $4 a$ & $\# 4$ & $34.1 \mathrm{~mm}$ & $2.7 \mathrm{~mm}$ & 15 & 5 & $\mathrm{CW}$ \\
\hline $4 \mathrm{~b}$ & $\# 4$ & $34.1 \mathrm{~mm}$ & $2.7 \mathrm{~mm}$ & 1 & 3 & $\mathrm{CW}$ \\
\hline $5 \mathrm{a}$ & $\# 4$ & $34.1 \mathrm{~mm}$ & $2.7 \mathrm{~mm}$ & 1 & 3 & $\mathrm{CW}$ \\
\hline $5 b$ & \#4 & $34.1 \mathrm{~mm}$ & $2.7 \mathrm{~mm}$ & 15 & 5 & $\mathrm{CW}$ \\
\hline $5 c$ & \#4 & $34.1 \mathrm{~mm}$ & $2.7 \mathrm{~mm}$ & 15 & 5 & $\mathrm{CCW}$ \\
\hline $6 \mathrm{a}$ & $\# 5$ & $30.1 \mathrm{~mm}$ & $2.6 \mathrm{~mm}$ & 15 & 5 & $\mathrm{CW}$ \\
\hline $6 \mathrm{~b}$ & $\# 5$ & $30.1 \mathrm{~mm}$ & $2.6 \mathrm{~mm}$ & 15 & 5 & $\mathrm{CCW}$ \\
\hline $6 c$ & $\# 5$ & $30.1 \mathrm{~mm}$ & $2.6 \mathrm{~mm}$ & 1 & 3 & $\mathrm{CW}$ \\
\hline $6 d$ & $\# 5$ & $30.1 \mathrm{~mm}$ & $2.6 \mathrm{~mm}$ & 1 & 3 & $\mathrm{CCW}$ \\
\hline $7 \mathrm{a}$ & $\# 5$ & $30.1 \mathrm{~mm}$ & $2.6 \mathrm{~mm}$ & 15 & 5 & $\mathrm{CW}$ \\
\hline $7 b$ & $\# 5$ & $30.1 \mathrm{~mm}$ & $2.6 \mathrm{~mm}$ & 15 & 5 & CCW \\
\hline $7 \mathrm{c}$ & $\# 5$ & $30.1 \mathrm{~mm}$ & $2.6 \mathrm{~mm}$ & 1 & 3 & $\mathrm{CW}$ \\
\hline $7 \mathrm{~d}$ & $\# 5$ & $30.1 \mathrm{~mm}$ & $2.6 \mathrm{~mm}$ & 1 & 3 & $\mathrm{CCW}$ \\
\hline $8 a$ & $\# 5$ & $30.1 \mathrm{~mm}$ & $2.6 \mathrm{~mm}$ & 1 & 3 & $\mathrm{CW}$ \\
\hline $8 \mathrm{~b}$ & $\# 5$ & $30.1 \mathrm{~mm}$ & $2.6 \mathrm{~mm}$ & 1 & 3 & CCW \\
\hline 9 & \#6 & $30.0 \mathrm{~mm}$ & $2.4 \mathrm{~mm}$ & 15 & 5 & $\mathrm{CW}$ \\
\hline 10 & $\# 6$ & $30.0 \mathrm{~mm}$ & $2.4 \mathrm{~mm}$ & 15 & 5 & $\mathrm{CCW}$ \\
\hline 11 & \#6 & $30.0 \mathrm{~mm}$ & $2.4 \mathrm{~mm}$ & 15 & 5 & $\mathrm{CW}$ \\
\hline 12 & $\# 7$ & $28.2 \mathrm{~mm}$ & $2.4 \mathrm{~mm}$ & 15 & 5 & $\mathrm{CCW}$ \\
\hline 13 & $\# 7$ & $28.2 \mathrm{~mm}$ & $2.4 \mathrm{~mm}$ & 15 & 5 & $\mathrm{CW}$ \\
\hline 14 & $\# 7$ & $28.2 \mathrm{~mm}$ & $2.4 \mathrm{~mm}$ & 15 & 5 & $\mathrm{CCW}$ \\
\hline 15 & $\# 7$ & $28.2 \mathrm{~mm}$ & $2.4 \mathrm{~mm}$ & 16 & 5 & CW \\
\hline
\end{tabular}




\subsection{Data Processing and Results}

The stored "raw" data record corresponded to a backscattered pressure wave resulting from a single insonification event (Fig. 3-10). Data runs 1 through 14 were attained with the incident pressure wave being produced by a narrowband signal. The scattered pressure wave was acquired within a period short enough so that only the backscattered signal from the animal was processed. The incident pressure wave had a sufficient pulse length to allow the backscattered signal from the animal to reach a steady-state. The steadystate echo allowed the insonification to be characterized by the maximum amplitude of the echo.

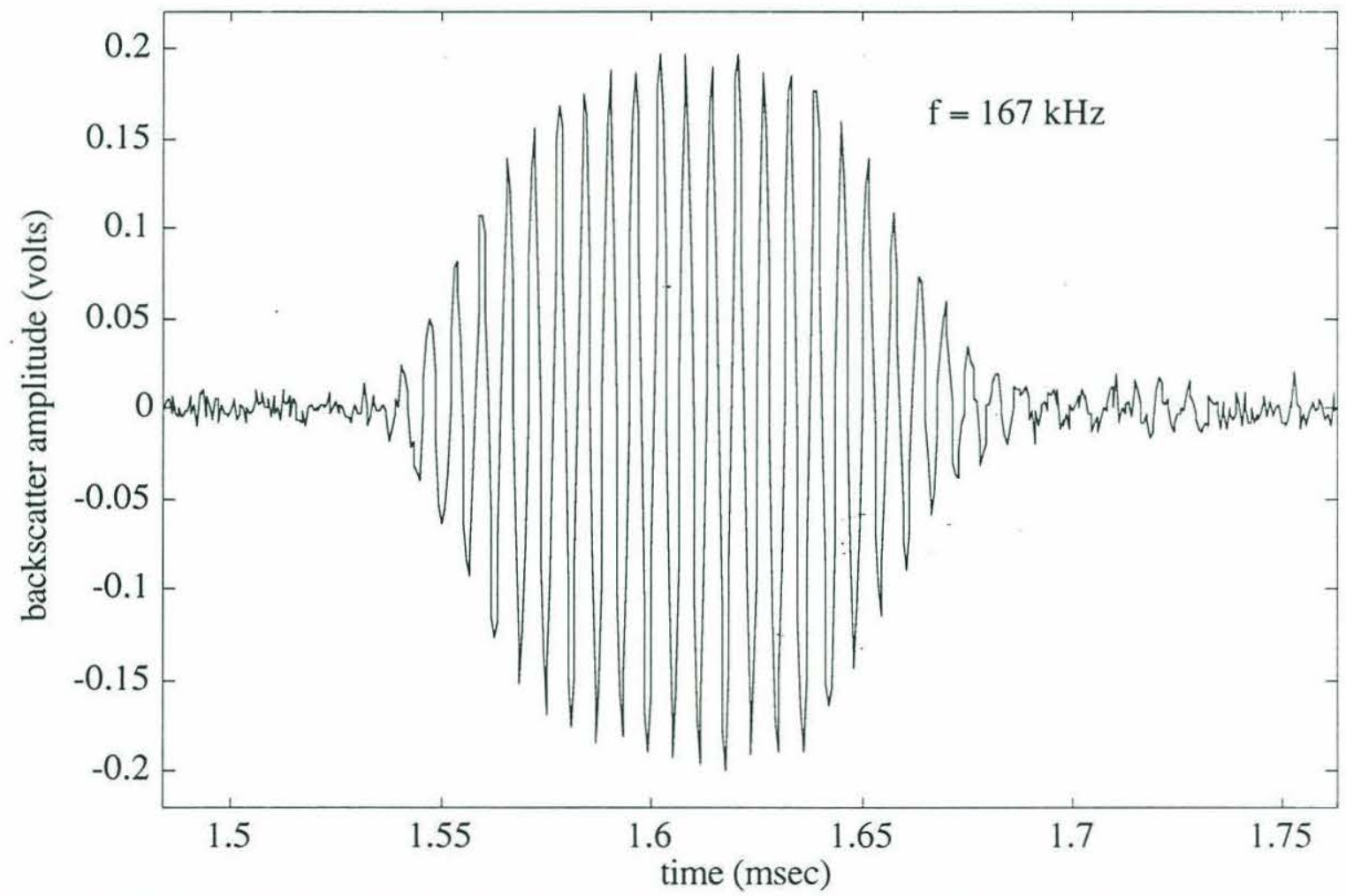

Figure 3-10: Typical backscattered pressure wave from a decapod shrimp. (This was collected at broadside orientation of an animal insonified at an acoustic frequency of $167 \mathrm{kHz}$. The horizontal scale is the time, in milliseconds, since the incident pressure wave was initiated. The incident pressure wave was a sinusoid with an amplitude of 2 volts, and a pulse length of $0.1 \mathrm{msec}$.) 
The amplitude was calculated by determining the maximum envelope of the time series. Each insonification event was thus characterized by a single number corresponding to the backscatter amplitude [21]:

$$
|\hat{\Phi}(\beta)|=\left|\frac{r \sqrt{\hat{p}_{s}^{2}(\mathbf{r})}}{A e^{i k_{m} r}}\right| .
$$

The data acquisition system measured the instantaneous value of the backscattered pressure (time-series) signal [31,32]. The backscatter cross section of each insonification event was calculated, and then the resultant points for each angular position were then averaged to give a mean backscatter cross section for a given orientation angle $[5,33,34]$. The peak backscatter cross section is related to the peak backscatter amplitude by [21]:

$$
\hat{\sigma}_{b s}(\beta) \propto|\hat{\Phi}(\beta)|^{2} .
$$

The mean backscatter cross section for a given orientation angle was determined by:

$$
\bar{\sigma}_{b s}(\beta)=\frac{1}{n} \sum_{i}^{n} \hat{\sigma}_{b s_{i}}(\beta),
$$

where $n$ is the number of insonification events at a given orientation angle.

For data run 15, a chirp signal was used to excite the broadband $500 \mathrm{kHz}$ transducer at a frequency range of 250 to $550 \mathrm{kHz}$. The backscattered pressure wave resulting from the chirp signal gave a continuous frequency response for the animal over the frequency range of the chirp. The disadvantage of using a chirp signal was that the energy at any given frequency was less than the energy for a narrowband signal at that frequency [35].

The data acquired from the chirp was processed by computing the Fourier transform of the backscattered pressure wave. The magnitude of Fourier transformed signal at a specific frequency and orientation was then squared and averaged. This produced equivalent results in the frequency domain as Eqs. (3.21) and (3.22) yielded in the time domain [36]. 
The resultant discrete values for mean backscatter cross section, from both the narrowband and broadband pressure waves, were fitted to a smooth curve by use of a cubic spline function [37]. The smoothed curves were then normalized to the maximum value of the backscatter cross section measured at each specific acoustic frequency, resulting in normalized backscatter directivity patterns for the decapod shrimp (Figs. 3-11 and 3-12).

Figure 3-11 presents the backscatter directivity plots for the narrowband data sets. It is arranged in order of increasing $k a$ and $L / \lambda$. The system noise for these data runs was less than the minimum averaged backscatter cross section of the animal, except for data set number 4 [Fig. 3-11(c,d)]. Thus, we had a measurable signal at all orientation angles for the remaining data sets; and since the shape of the backscatter directivity patterns was derived by comparing measurable signals, system noise was not a factor in these data sets. On data set number 4 system noise was approximately 20 percent of the peak signal, and masked the signals at orientation angles other than the broadside aspects. This could have caused a widening of the experimentally measured main lobe.

From the normalized backscatter directivity patterns, the broadside orientation beamwidths were measured. These beamwidths were the same beamwidths defined in chapter 2: the angle subtended between the smallest and largest angles at which the backscatter cross section reaches one-half the maximum value of the backscatter cross section. Table 3-8 lists the experimentally measured beamwidths from the narrowband analysis. Some of the narrowband data sets exhibited irregular spikes [data sets: 4a, 4b, 3, 14, 13; Fig. 3-11(c,d,j,q,t)]. These spikes produced unnaturally large beamwidths if the definition in chapter 2 was followed blindly. For these data sets, the beamwidth was measured conservatively as the author's "best guess" of the boundaries of the sometimes jagged main lobe. The "recorded" beamwidths are shown on Fig. 3-11 as horizontal chords. Due to the above use of the author's "artistic license", Fig. 3-11 includes most data to archive the results and allow the reader to judge the validity of assumptions. 

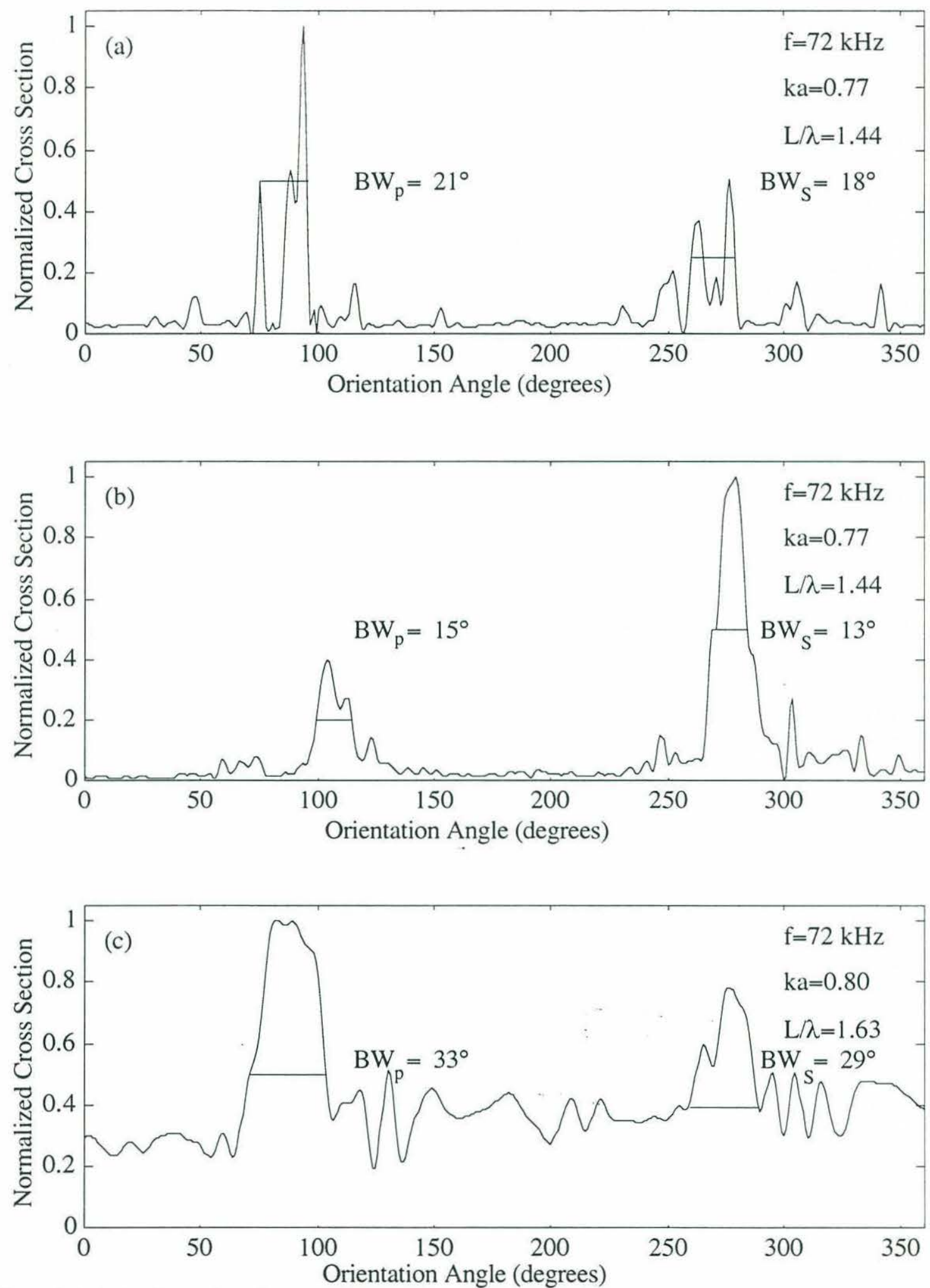

Figure 3-11(a-c): Examples of measured backscatter directivity patterns for decapod shrimp. [Top, data set number 8a (fast angular sweep), animal \#5; middle, data set number 8b (fast angular sweep), animal \#5; bottom, data set number $4 \mathrm{a}$ (slow angular sweep), animal \#4.] 

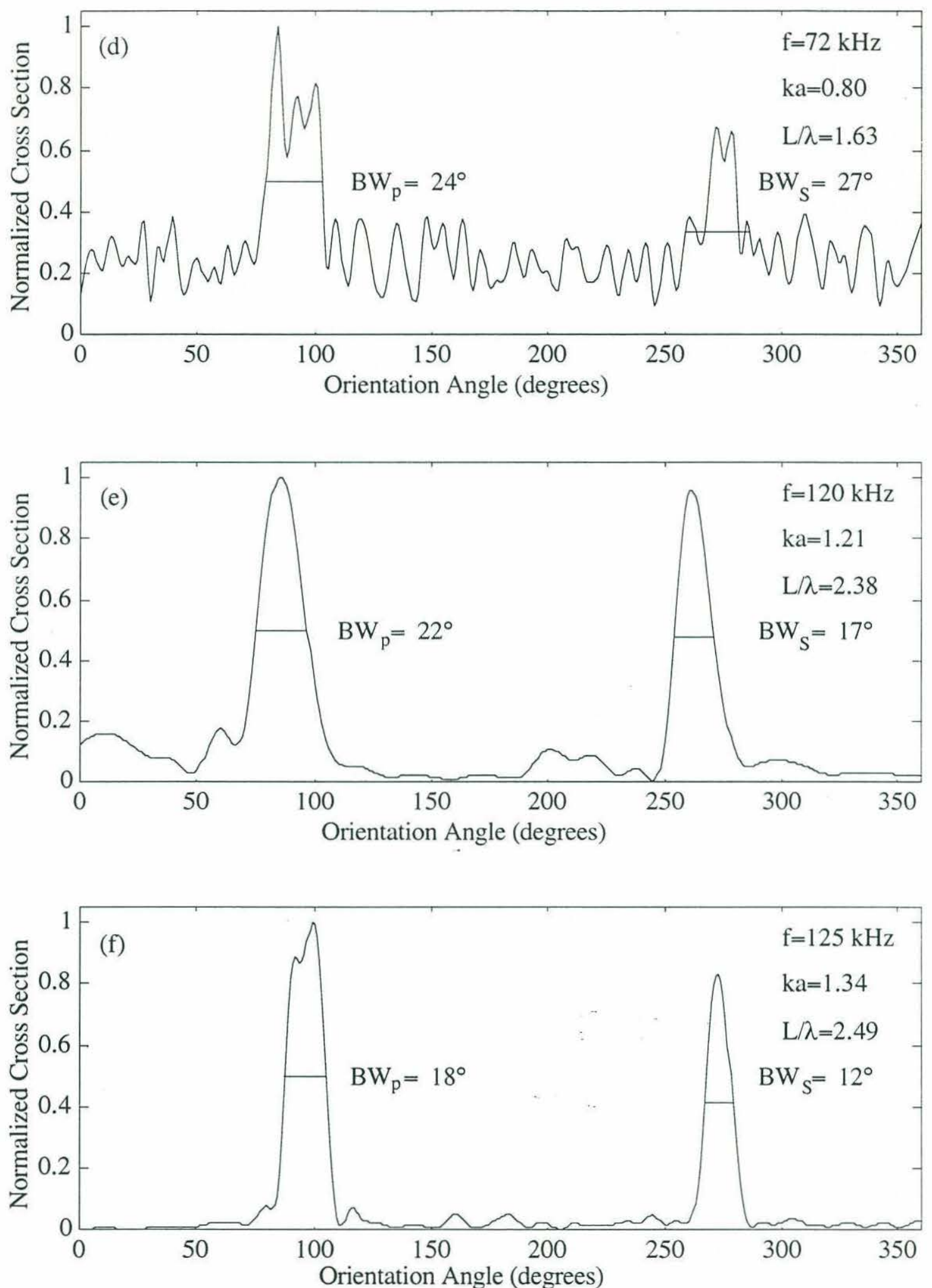

Figure 3-11(d-f): Examples of measured backscatter directivity patterns for decapod shrimp. [Top, data set number 4b (fast angular sweep), animal \#4; middle, data set number 1 (slow angular sweep), animal \#1; bottom, data set number 7a (slow angular sweep), animal \#5.] 

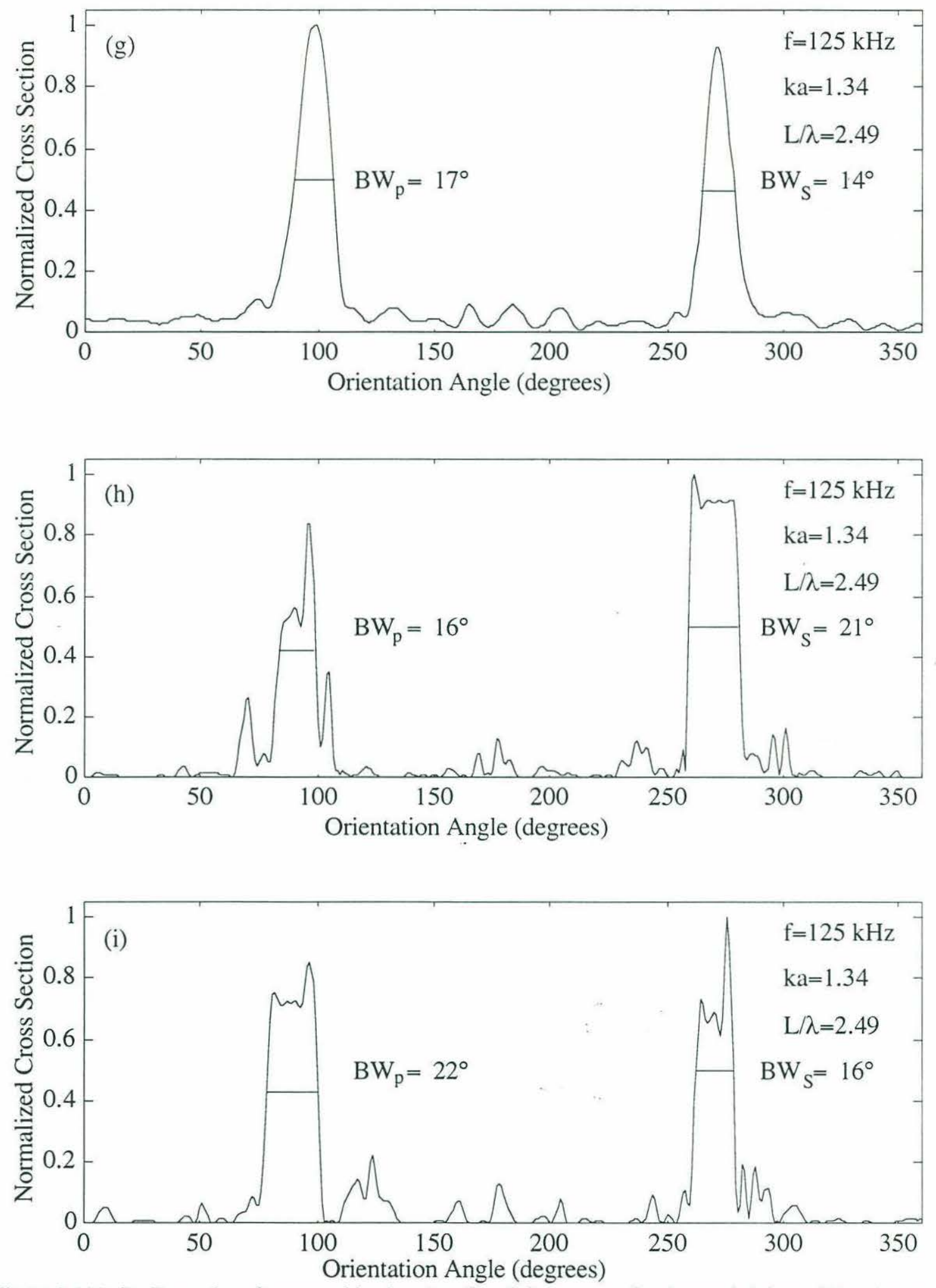

Figure 3-11(g-i): Examples of measured backscatter directivity patterns for decapod shrimp. [Top, data set number $7 \mathrm{~b}$ (slow angular sweep), animal \#5; middle, data set number 7c (fast angular sweep), animal \#5: bottom, data set number 7d (fast angular sweep), animal \#5.] 

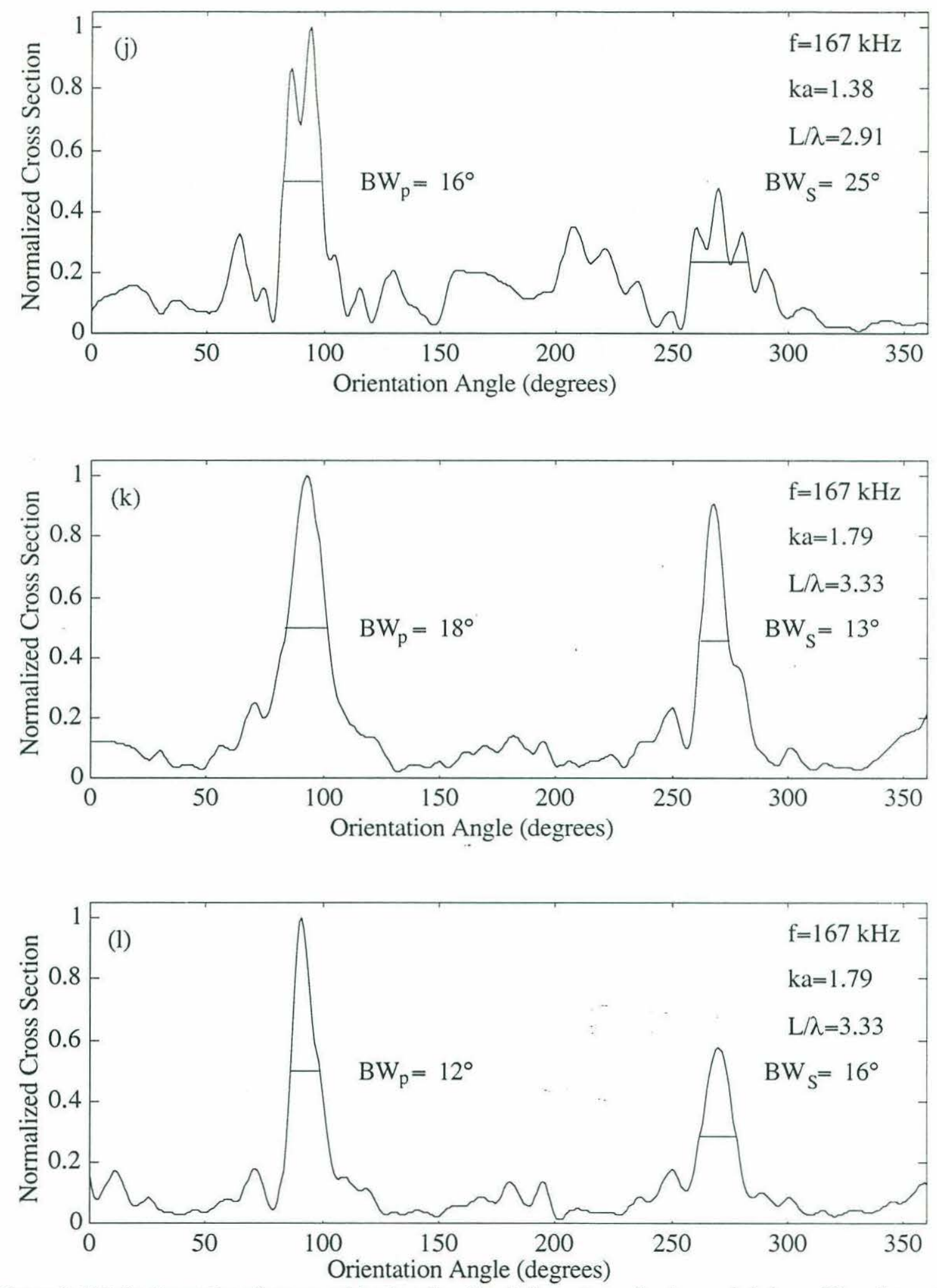

Figure 3-11(j-1): Examples of measured backscatter directivity patterns for decapod shrimp. [Top, data set number 3 (slow angular sweep), animal \#3; middle, data set number 6a (slow angular sweep), animal \#5; bottom, data set number $6 \mathrm{~b}$ (slow angular sweep), animal \#5.] 

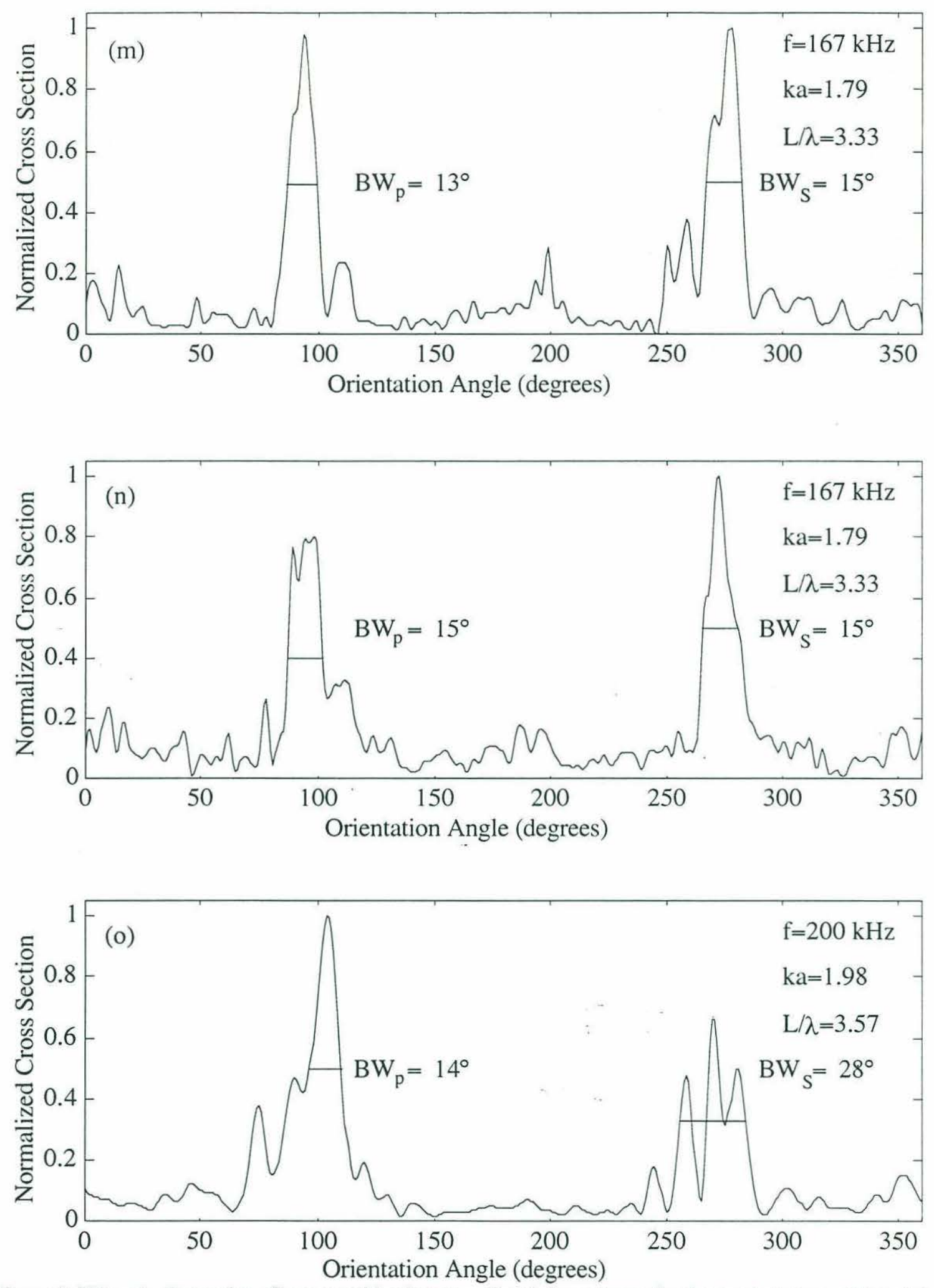

Figure 3-11(m-o): Examples of measured backscatter directivity patterns for decapod shrimp. [Top, data set number 6c (fast angular sweep), animal \#5; middle, data set number 6d (fast angular sweep), animal \#5; bottom, data set number 2 (slow angular sweep), animal \#2.] 

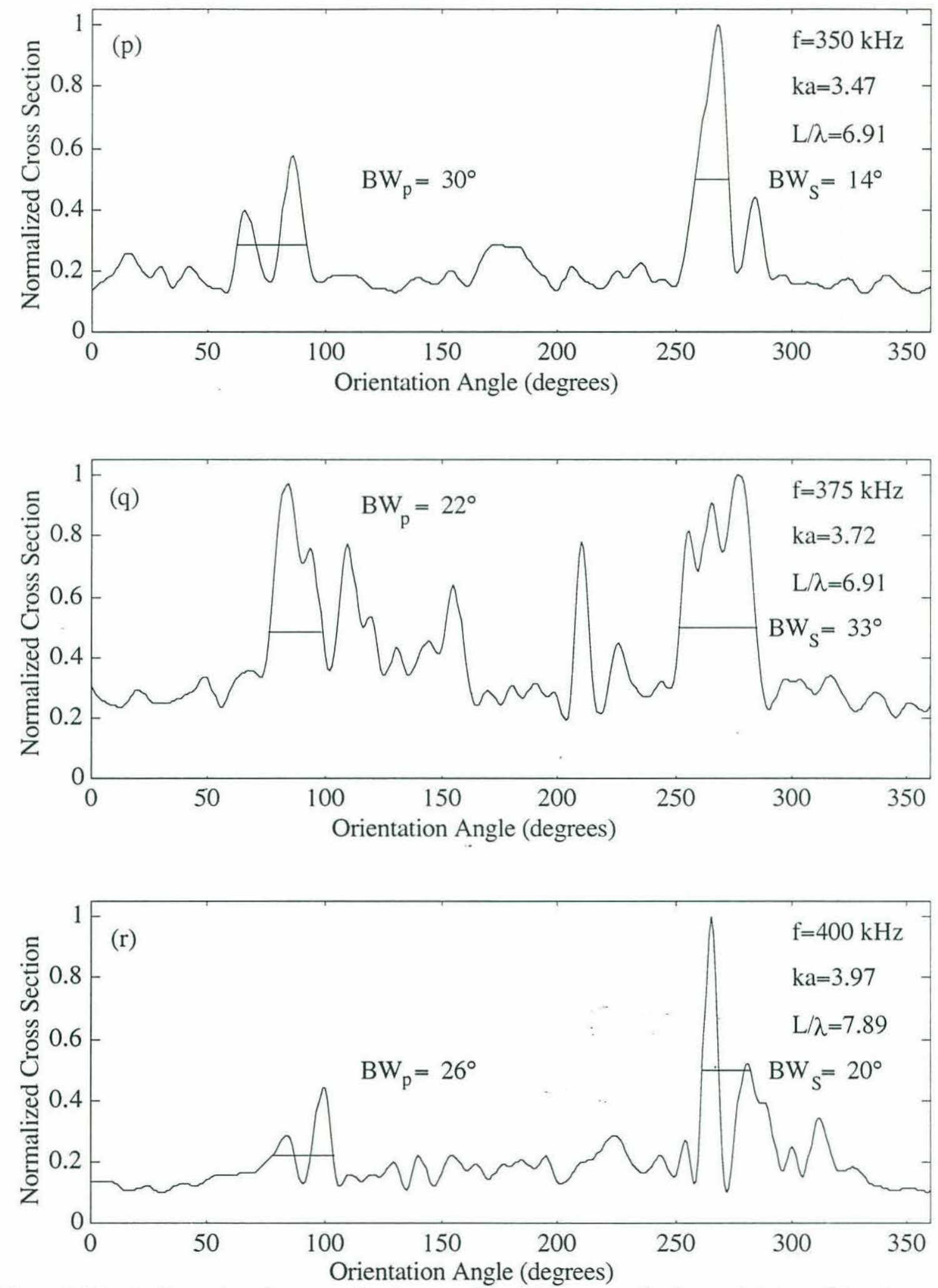

Figure 3-11(p-r): Examples of measured backscatter directivity patterns for decapod shrimp. [Top, data set number 9 (slow angular sweep), animal \#6; middle, data set number 14 (slow angular sweep), animal \#7; bottom, data set number 10 (slow angular sweep), animal \#6.] 

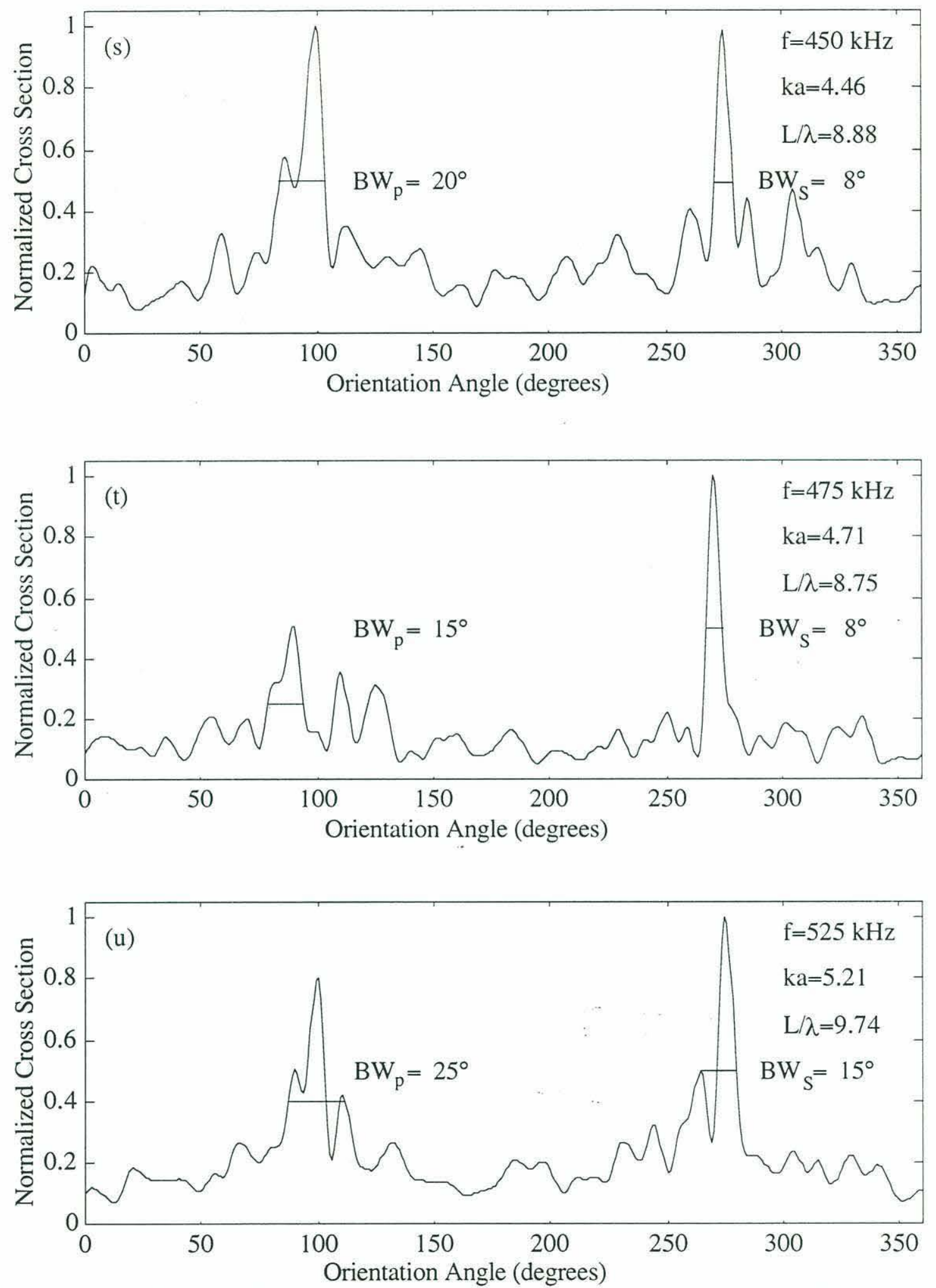

Figure 3-11(s-u): Examples of measured backscatter directivity patterns for decapod shrimp. [Top, data set number 11 (slow angular sweep), animal \#6; middle, data set number 13 (slow angular sweep), animal \#7; bottom, data set number 12 (slow angular sweep), animal \#7.] 
Table 3-8.- Experimentally Measured Beamwidths from Narrowband Analyses

$\begin{array}{ccccccc}\begin{array}{c}\text { Data Set } \\ \text { Number }\end{array} & \text { Animal } & \begin{array}{c}\text { Transducer } \\ \text { Frequency }\end{array} & k a & L / \lambda & \begin{array}{c}\text { Port } \\ \text { Beamwidth }\end{array} & \begin{array}{c}\text { Starboard } \\ \text { Beamwidth }\end{array} \\ 1 & \# 1 & 120 \mathrm{kHz} & 1.21 & 2.38 & 22^{\circ} & 17^{\circ} \\ 2 & \# 2 & 200 \mathrm{kHz} & 1.98 & 3.56 & 14^{\circ} & 28^{\circ} \\ 3 & \# 3 & 167 \mathrm{kHz} & 1.38 & 2.91 & 16^{\circ} & 25^{\circ} \\ 4 \mathrm{a} & \# 4 & 72 \mathrm{kHz} & 0.80 & 1.63 & 33^{\circ} & 33^{\circ} \\ 4 \mathrm{~b} & \# 4 & 72 \mathrm{kHz} & 0.80 & 1.63 & 24^{\circ} & 27^{\circ} \\ 5 \mathrm{a} & \# 4 & 200 \mathrm{kHz} & 2.23 & 4.52 & 18^{\circ} & 18^{\circ} \\ 5 \mathrm{~b} & \# 4 & 200 \mathrm{kHz} & 2.23 & 4.52 & 20^{\circ} & 23^{\circ} \\ 5 \mathrm{c} & \# 4 & 200 \mathrm{kHz} & 2.23 & 4.52 & 16^{\circ} & 27^{\circ} \\ 6 \mathrm{a} & \# 5 & 167 \mathrm{kHz} & 1.79 & 3.33 & 18^{\circ} & 13^{\circ} \\ 6 \mathrm{~b} & \# 5 & 167 \mathrm{kHz} & 1.79 & 3.33 & 12^{\circ} & 16^{\circ} \\ 6 \mathrm{c} & \# 5 & 167 \mathrm{kHz} & 1.79 & 3.33 & 13^{\circ} & 15^{\circ} \\ 6 \mathrm{~d} & \# 5 & 167 \mathrm{kHz} & 1.79 & 3.33 & 15^{\circ} & 15^{\circ} \\ 7 \mathrm{a} & \# 5 & 125 \mathrm{kHz} & 1.34 & 2.49 & 18^{\circ} & 12^{\circ} \\ 7 \mathrm{~b} & \# 5 & 125 \mathrm{kHz} & 1.34 & 2.49 & 17^{\circ} & 14^{\circ} \\ 7 \mathrm{c} & \# 5 & 125 \mathrm{kHz} & 1.34 & 2.49 & 16^{\circ} & 21^{\circ} \\ 7 \mathrm{~d} & \# 5 & 125 \mathrm{kHz} & 1.34 & 2.49 & 16^{\circ} & 22^{\circ} \\ 8 \mathrm{a} & \# 5 & 72 \mathrm{kHz} & 0.77 & 1.44 & 21^{\circ} & 19^{\circ} \\ 8 \mathrm{~b} & \# 5 & 72 \mathrm{kHz} & 0.77 & 1.44 & 16^{\circ} & 17^{\circ} \\ 9 & \# 6 & 350 \mathrm{kHz} & 3.47 & 6.91 & 30^{\circ} & 14^{\circ} \\ 10 & \# 6 & 400 \mathrm{kHz} & 3.97 & 7.89 & 26^{\circ} & 20^{\circ} \\ 11 & \# 6 & 450 \mathrm{kHz} & 4.46 & 8.88 & 20^{\circ} & 8^{\circ} \\ 12 & \# 7 & 525 \mathrm{kHz} & 5.21 & 9.67 & 25^{\circ} & 17^{\circ} \\ 13 & \# 7 & 475 \mathrm{kHz} & 4.71 & 8.75 & 15^{\circ} & 8^{\circ} \\ 14 & \# 7 & 375 \mathrm{kHz} & 3.72 & 6.91 & 22^{\circ} & 33^{\circ}\end{array}$


Figure 3-12 shows the results of the data from the broadband chirp for a slow angular sweep at acoustic frequencies of 320 to $487 \mathrm{kHz}$ in steps of $6.7 \mathrm{kHz}$. Table 3-9 lists the results from the broadband chirp. As discussed earlier in this section, the broadband chirp gave a range of frequencies at the expense of a lower energy at any given frequencies. This lower energy, coupled with animal motion, lead to the port aspect producing no useful data. It is believed that the backscatter directivity pattern was similar to the narrowband backscatter directivity pattern illustrated in Fig. 3-11(r) and at the lower energy of the incident pressure wave, no useful signal was received from the port aspect. Thus, only the starboard aspect is depicted in Fig. 3-12 and enumerated in Table 3-9. (Note, in comparing Figs. 3-11 and 3-12, the horizontal axis of Fig. 3-12 is such that $0^{\circ}$ corresponds to the data from the telson aspect and $180^{\circ}$ corresponds to the head aspect data; whereas the horizontal axis of Fig. 3-11 is such that at $0^{\circ}$ and $360^{\circ}$ correspond to the data from the head aspect and $180^{\circ}$ to data from the telson aspect data.)

Observing Fig. 3-12, two chords representing beamwidth are presented. They are separated vertically for illustrative purposes only. The upper one shows the "no rogue" based beamwidth $\left(\mathrm{BW}_{\mathrm{N}}\right)$ that ignores a certain "rogue" side lobe that reaches 50 percent of the maximum cross section at an acoustic frequency on $420 \mathrm{kHz}$ [Fig. 3-12(p)]. This side lobe does not appear to be part of the main lobe, as it drifts away from the main lobe as the frequency is increased [Fig. 3-12(p-z)], instead of degenerating into the main lobe as would be expected from theory (Fig. 2-6). The lower chord is the "rogue" based beamwidth $\left(\mathrm{BW}_{\mathrm{R}}\right)$ that includes this side lobe. Inclusion or exclusion of this side lobe is purely subjective, hence both sets of estimates are given .

Similar experiments were conducted by Greenlaw [15] in 1990 over a frequency range of 190 to $230 \mathrm{kHz}$ on live and preserved euphausiids (Euphausia pacifica) at single frequencies. This animal is a species of elongated zooplankton similar in shape to the decapod shrimp used in this thesis. Greenlaw's experiments presented the half-power beamwidth as a function of $L / \lambda$. The corresponding values of $k a$ were found by using 
the $L / a$ ratio for Euphausia pacifica. For these animals, $L / a \approx 14$ [38]. These results cover a smaller frequency range than the data listed previously, but the sixteen data act as an independent check on a subset of the experimentally determined points found in this thesis.

Table 3-9. - Experimentally Measured Beamwidths from Broadband Analyses

(Data Set Number 15, Animal \#7)

\begin{tabular}{|c|c|c|c|c|}
\hline Frequency & $k a$ & $L / \lambda$ & $\begin{array}{l}\text { "No rogue" } \\
\text { based } \\
\text { Beamwidth }\end{array}$ & $\begin{array}{l}\text { "Rogue" } \\
\text { based } \\
\text { Beamwidth }\end{array}$ \\
\hline $320 \mathrm{kHz}$ & 3.17 & 5.94 & $10^{\circ}$ & $10^{\circ}$ \\
\hline $327 \mathrm{kHz}$ & 3.24 & 6.06 & $10^{\circ}$ & $10^{\circ}$ \\
\hline $333 \mathrm{kHz}$ & 3.31 & 6.18 & $8^{\circ}$ & $8^{\circ}$ \\
\hline $340 \mathrm{kHz}$ & 3.37 & 6.31 & $9^{\circ}$ & $9^{\circ}$ \\
\hline $347 \mathrm{kHz}$ & 3.43 & 6.43 & $26^{\circ}$ & $26^{\circ}$ \\
\hline $353 \mathrm{kHz}$ & 3.51 & 6.56 & $25^{\circ}$ & $25^{\circ}$ \\
\hline $360 \mathrm{kHz}$ & 3.57 & 6.68 & $24^{\circ}$ & $24^{\circ}$ \\
\hline $367 \mathrm{kHz}$ & 3.64 & 6.80 & $28^{\circ}$ & $28^{\circ}$ \\
\hline $373 \mathrm{kHz}$ & 3.70 & 6.93 & $27^{\circ}$ & $27^{\circ}$ \\
\hline $380 \mathrm{kHz}$ & 3.77 & 7.05 & $25^{\circ}$ & $25^{\circ}$ \\
\hline $387 \mathrm{kHz}$ & 3.84 & 7.17 & $25^{\circ}$ & $25^{\circ}$ \\
\hline $393 \mathrm{kHz}$ & 3.90 & 7.30 & $26^{\circ}$ & $26^{\circ}$ \\
\hline $400 \mathrm{kHz}$ & 3.97 & 7.42 & $25^{\circ}$ & $25^{\circ}$ \\
\hline $407 \mathrm{kHz}$ & 4.03 & 7.54 & $24^{\circ}$ & $24^{\circ}$ \\
\hline $413 \mathrm{kHz}$ & 4.10 & 7.67 & $24^{\circ}$ & $24^{\circ}$ \\
\hline $420 \mathrm{kHz}$ & 4.17 & 7.79 & $21^{\circ}$ & $31^{\circ}$ \\
\hline $427 \mathrm{kHz}$ & 4.23 & 7.92 & $19^{\circ}$ & $33^{\circ}$ \\
\hline $433 \mathrm{kHz}$ & 4.30 & 8.04 & $19^{\circ}$ & $34^{\circ}$ \\
\hline $440 \mathrm{kHz}$ & 4.37 & 8.16 & $18^{\circ}$ & $33^{\circ}$ \\
\hline $447 \mathrm{kHz}$ & 4.43 & 8.29 & $17^{\circ}$ & $35^{\circ}$ \\
\hline $453 \mathrm{kHz}$ & 4.50 & 8.41 & $17^{\circ}$ & $37^{\circ}$ \\
\hline $460 \mathrm{kHz}$ & 4.56 & 8.53 & $17^{\circ}$ & $38^{\circ}$ \\
\hline $467 \mathrm{kHz}$ & 4.63 & 8.66 & $17^{\circ}$ & $40^{\circ}$ \\
\hline $473 \mathrm{kHz}$ & 4.70 & 8.78 & $16^{\circ}$ & $39^{\circ}$ \\
\hline $480 \mathrm{kHz}$ & 4.76 & 8.91 & $16^{\circ}$ & $40^{\circ}$ \\
\hline $487 \mathrm{kHz}$ & 4.83 & 9.03 & $16^{\circ}$ & $40^{\circ}$ \\
\hline
\end{tabular}



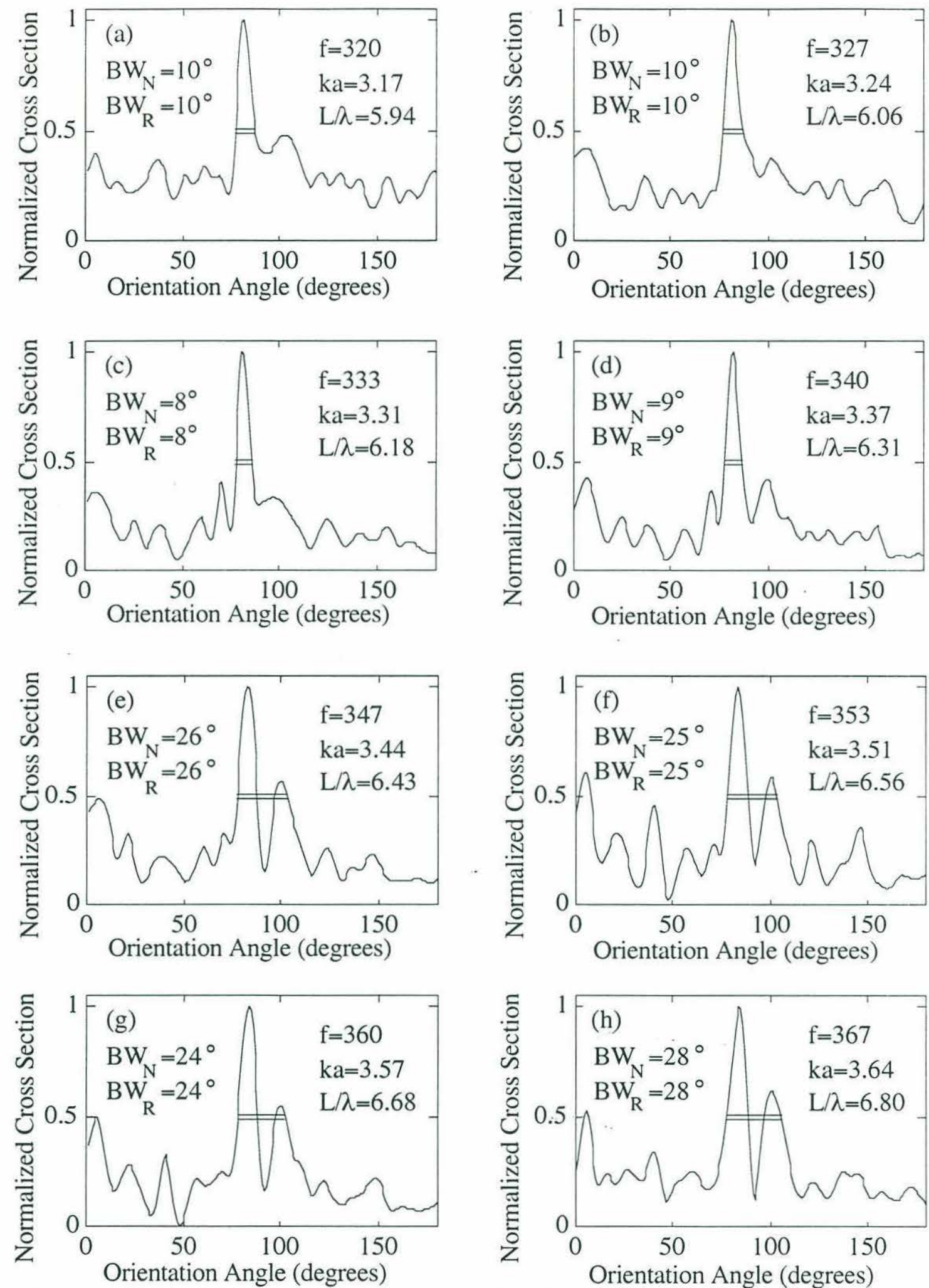

Figure 3-12(a-h): Directivity patterns from "chirp" data. This is the starboard aspect of the animal, with the head at $180^{\circ}$ and the telson at $0^{\circ}$. (Note the dramatic increase in beamwidth between acoustic frequencies of 340 and $347 \mathrm{kHz}$.) 

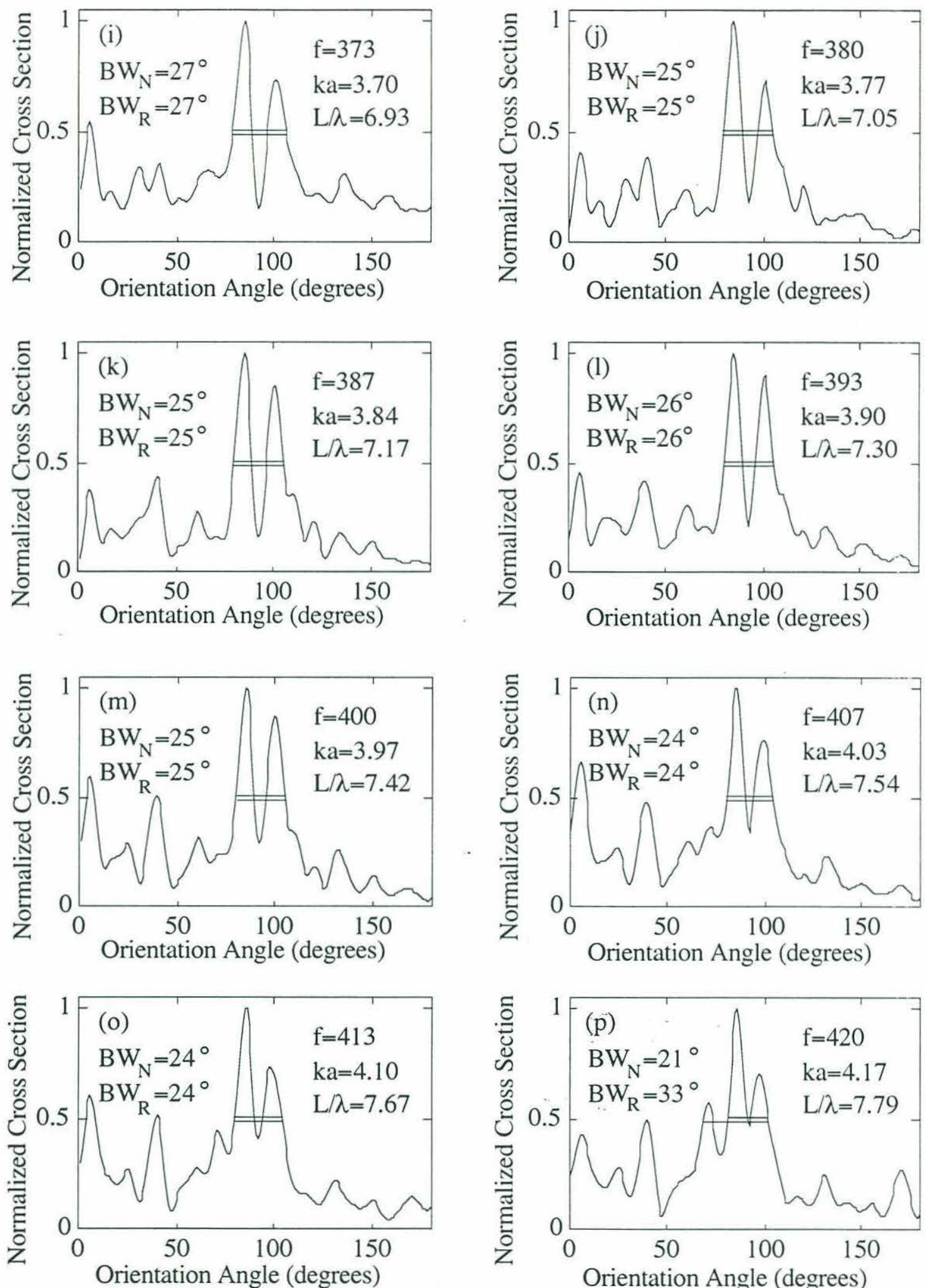

Figure 3-12(i-p): Directivity patterns from "chirp" data. This is the starboard aspect of the animal, with the head at $180^{\circ}$ and the telson at $0^{\circ}$. (Note the growth of the "rogue" side lobe at acoustic frequency of $420 \mathrm{kHz}$.) 

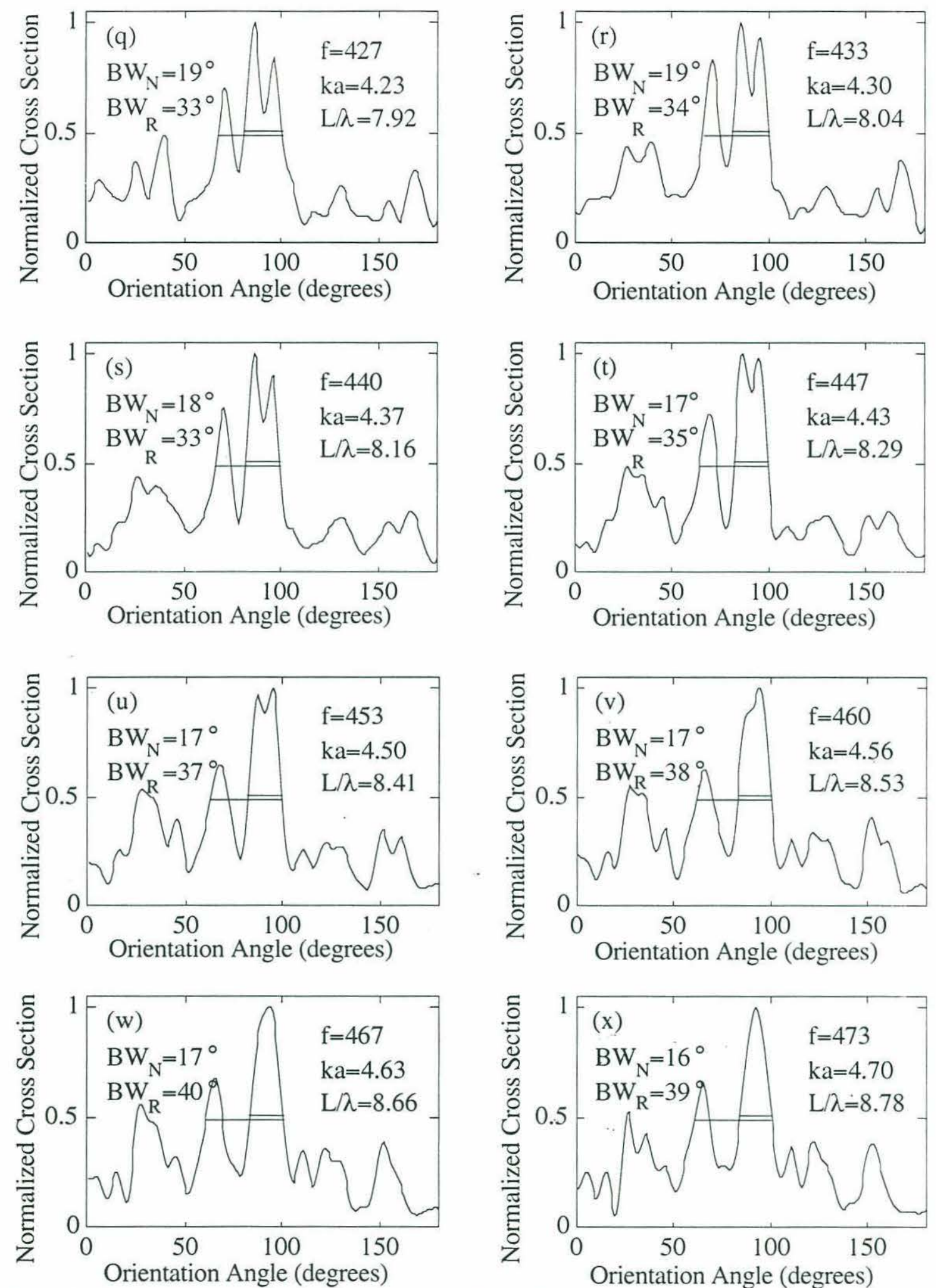

Figure 3-12(q-x): Directivity patterns from "chirp" data. This is the starboard aspect of the animal, with the head at $180^{\circ}$ and the telson at $0^{\circ}$. (Observe the increased separation of the "rogue" side lobe away from the center lobe.) 

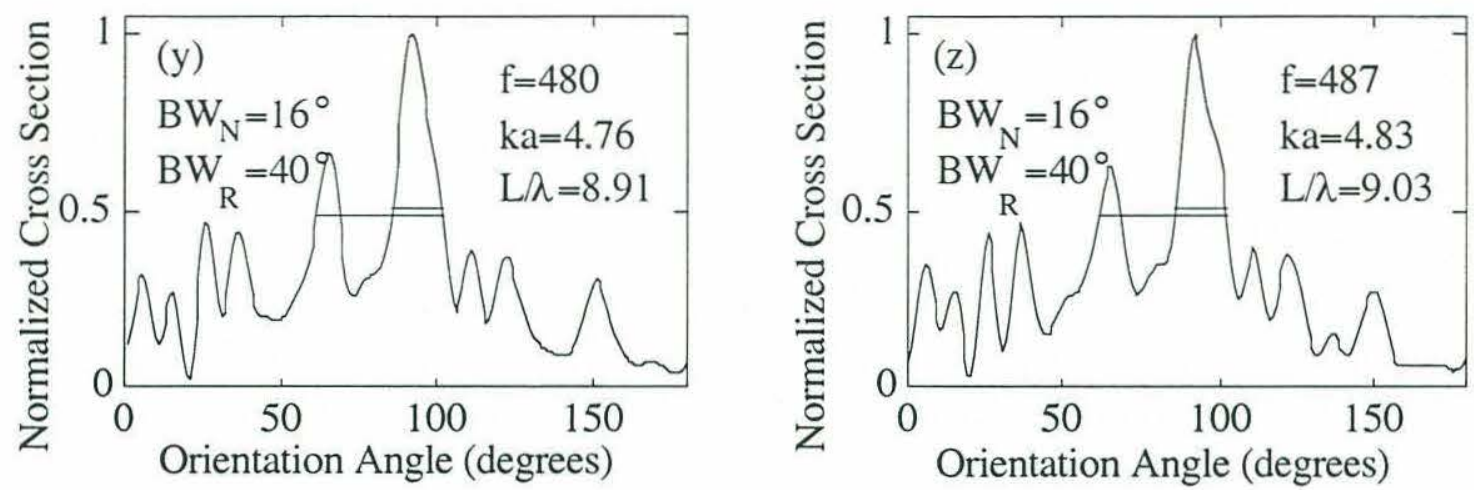

Figure 3-12(y,z): Directivity patterns from "chirp" data. This is the starboard aspect of the animal, with the head at $180^{\circ}$ and the telson at $0^{\circ}$.

Table 3-10.- Data from Greenlaw's Measurements of Euphausia pacifica (1990)

Data Set Number

$\begin{array}{cccc}1 & 0.83 & 1.86 & 17.5^{\circ} \\ 2 & 0.97 & 2.16 & 16.5^{\circ} \\ 3 & 1.03 & 2.30 & 15.2^{\circ} \\ 4 & 1.03 & 2.30 & 15.0^{\circ} \\ 5 & 1.09 & 2.43 & 16.8^{\circ} \\ 6 & 1.14 & 2.53 & 16.5^{\circ} \\ 7 & 1.14 & 2.53 & 14.0^{\circ} \\ 8 & 1.19 & 2.65 & 15.2^{\circ} \\ 9 & 1.19 & 2.65 & 15.0^{\circ} \\ 10 & 1.19 & 2.65 & 14.5^{\circ} \\ 11 & 1.21 & 2.70 & 12.0^{\circ} \\ 12 & 1.25 & 2.78 & 12.5^{\circ} \\ 13 & 1.31 & 2.91 & 14.5^{\circ} \\ 14 & 1.31 & 2.91 & 11.2^{\circ} \\ 15 & 1.37 & 3.05 & 12.0^{\circ} \\ 16 & 1.37 & 3.05 & 11.0^{\circ}\end{array}$




\section{Chapter 4}

\section{Synthesis and Discussion}

Figure 4-1 shows the experimental data from Tables 3-8, 3-9 and 3-10 plotted with theoretical prediction of beamwidth versus $k a$ and $L / \lambda$ (Fig. 2-12). The mean theoretical curve is plotted as a solid line (-), while the dashed lines (- -) represent the beamwidths that fall within one standard deviation of the mean of the one hundred trial runs performed. The narrowband data sets are denoted by a circle ('o'); the broadband chirp results are denoted by a cross within a circle (“ $\oplus$ ’) for the "no rogue” data, or an asterisk ('*’) for the "rogue" data; and the results from Greenlaw's experiments are denoted by a cross ('+'). Most data generally follow the predicted trends of the theoretical beamwidth.

The experimentally measured beamwidths tend to decrease monotonically for values of $k a$ less than 1.9 and $L / \lambda$ less than 3.4. Beyond those points the beamwidths oscillate, more-or-less about a mean value of approximately $19^{\circ}$.

The narrowband data set number $2(k a=1.98$ and $L / \lambda=3.56)$ shows the first step increment in beamwidth. The results from the broadband chirp signal show quite clearly a second step increase at $k a=3.4(L / \lambda=6.5)$. These values of $k a$ correspond to the nulls in target strength versus $k a$ plots for elongated zooplankton [2,4]. After this second step increase, the "no rogue"-based beamwidths generally decrease with increasing acoustic frequency. This local monotonic decrease is supported by the narrowband data collected in this region, until the $525 \mathrm{kHz}$ data shows an increasing trend in beamwidth $(k a=5.2$, $L / \lambda=9.7)$. Conversely, at $k a=4.2(L / \lambda=7.8)$ the "rogue"-based beamwidths increase with acoustic frequency to a value of around $40^{\circ}$. As discussed before, the effect is due to 
the definition of the main lobe, something that is subjective with these types of patterns. While this author believes that the rogue side lobe is not associated with the main lobe, its effect on the directivity pattern should not be ignored.

The narrowband data gathered at 400,450 , and $475 \mathrm{kHz}$ show a decreasing trend in beamwidth. Yet, observing Fig. 3-11(s,t) there is evidence of a rogue side lobe (smaller than that observed in the chirp echoes) that could potentially increase the beamwidth. Thus the prolate spheroid model for elongated zooplankton is apparently valid up to values of $k a \approx 4$ and $L / \lambda \approx 8$, beyond which echo features from the animal's microstructure and the definition used for beamwidth cause the experimental data to deviate from the simplified theoretical predictions.

In addition to the beamwidth, the level of the side lobes had some interesting properties. From Fig. 3-11 it is noted that the side lobes of the backscatter directivity pattern for the decapod shrimp had a magnitude in the range of approximately 5 percent of the peak value of the main lobe at lower acoustic frequencies ( 72 and $125 \mathrm{kHz}$ ), 5 to 10 percent of the peak value of the main lobe at the middle acoustic frequencies (167 and $200 \mathrm{kHz}$ ), and 10 to 20 percent of the peak value of the main lobe at higher acoustic frequencies (350 to $525 \mathrm{kHz}$ ). These average side lobe levels will be referred to as the pedestal of the backscatter (cross section) directivity pattern. (Independent experiments by Greenlaw also showed approximately the same corresponding peak-to-pedestal ratio at $200 \mathrm{kHz}$ [15].) Figure 2-11 shows that the pedestal of backscatter directivity pattern of the prolate spheroid was smaller.

The maximum normalized target strength at end-on incidence on the prolate spheroid is plotted in Fig. 4-2. End-on incidence is being defined as orientation angles $(\beta)$ between $0^{\circ}$ and $10^{\circ}$. The target strength is presented in decibels (dB), where [39]:

$$
1 \mathrm{~dB}=10 \log _{10}\left(\frac{\sigma_{b s}}{\sigma_{b s_{\max }}}\right) .
$$



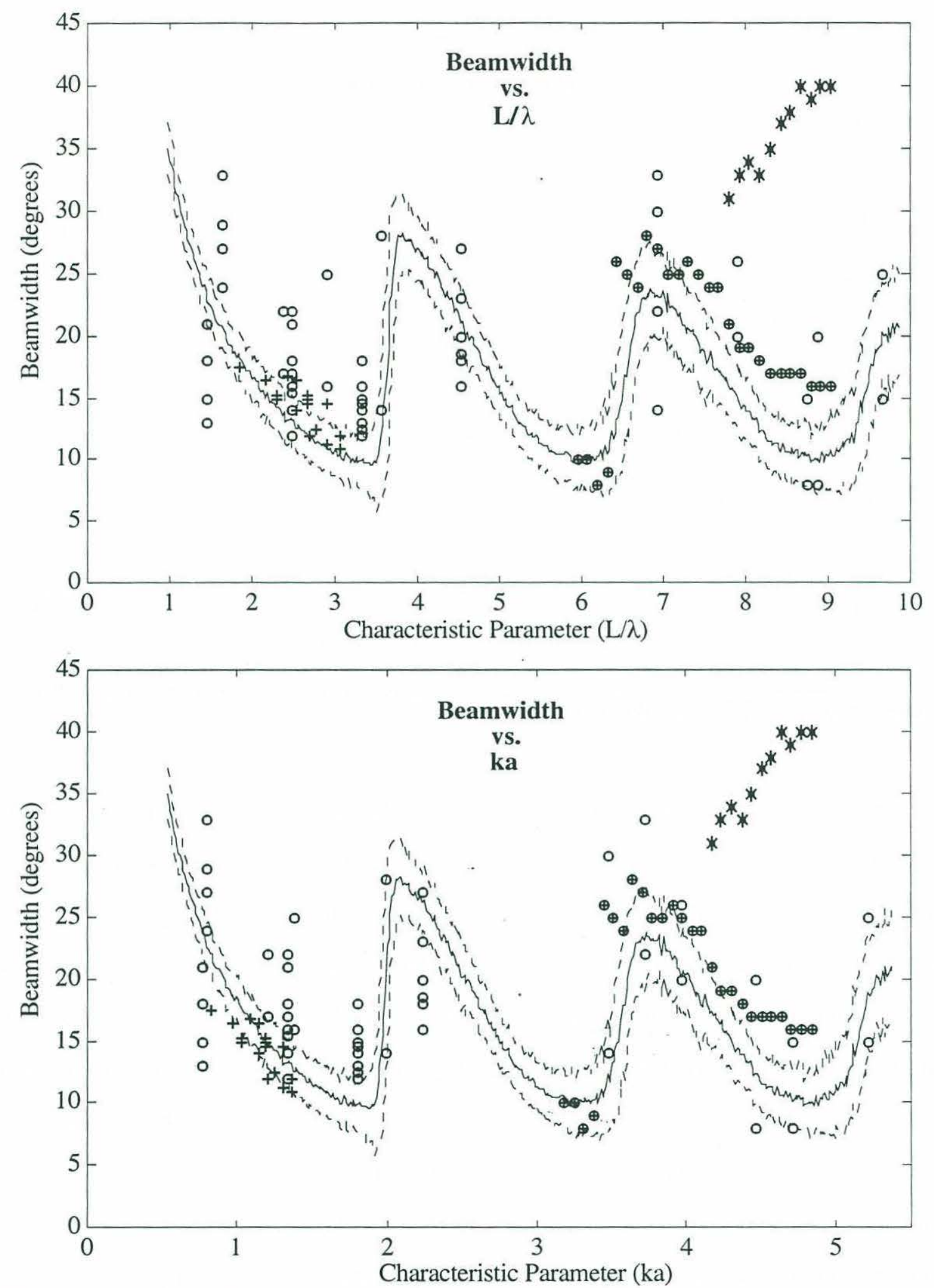

Figure 4-1: Experimentally and theoretically determined beamwidths plotted against the characteristic parameters, $k a$ and $L / \lambda$. ('o' denotes narrowband data; ' $*$ ' and ' $\oplus$ ' denote chirp data, the ' $*$ ' data involves including a "rogue" side lobe in the estimate of beamwidth; and 't' denotes Greenlaw's data. The theoretical data has modeled animal motion and sampling limitations.) 


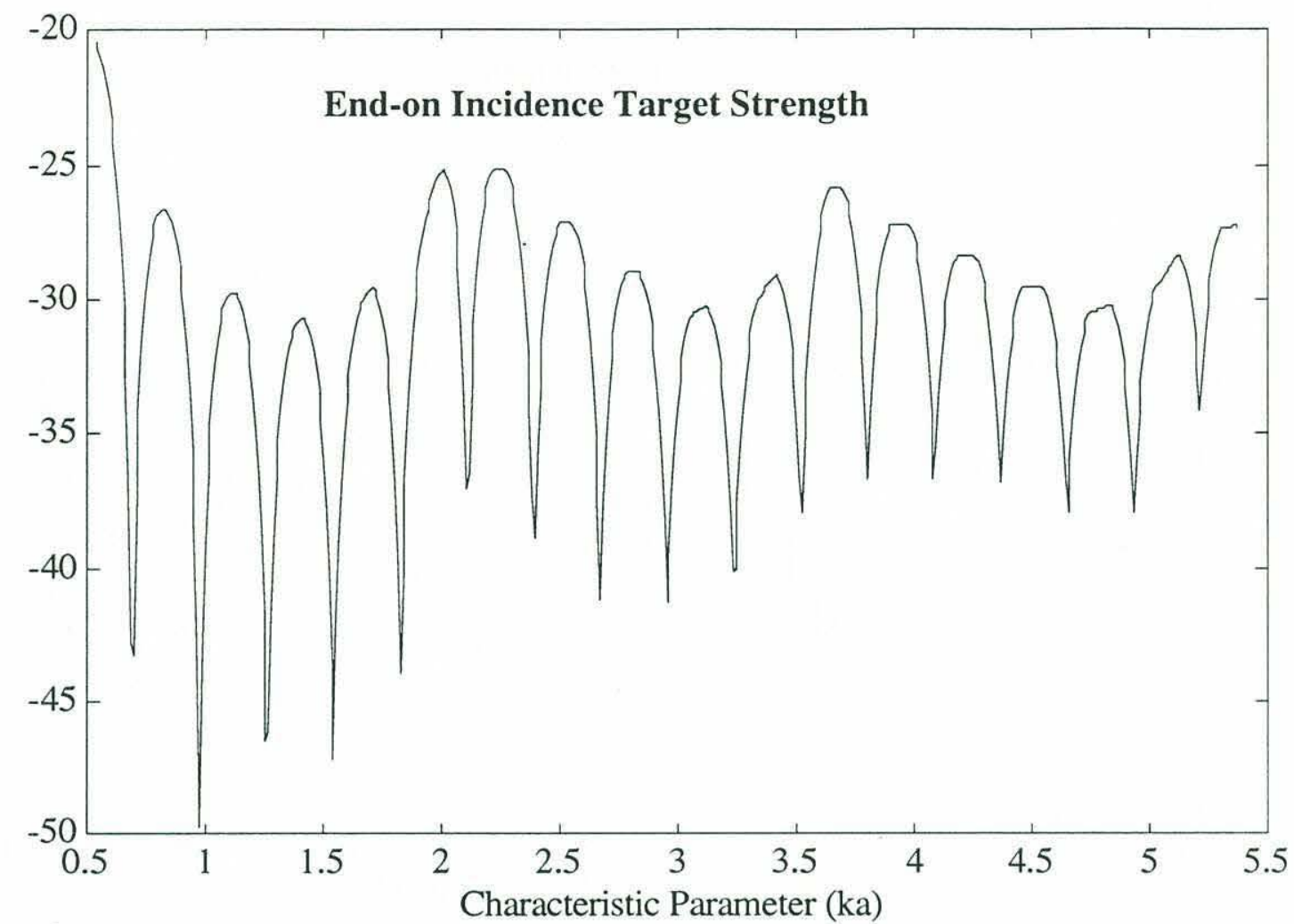

Figure 4-2: Theoretical backscatter target strength near end-on incidences. (The normalized target strength is the maximum target strength for orientation angles between $0^{\circ}$ and $10^{\circ}$. It is normalized to the maximum target strength of the backscatter directivity pattern.)

The model predicts a pedestal of 0.1 to 1 percent of the maximum backscatter cross section $(-30$ to $-20 \mathrm{~dB})$. This is one or two orders of magnitude less than the pedestal found in the experimentally derived backscatter directivity patterns of the decapod shrimp. This difference is possibly due to the failure of the model to account for backscatter from the microstructures on the animal, such as the telson, pleopods and maxilla [1]. This assumption is supported by the fact that the magnitude of the pedestals observed in Fig. 3-11 increased with increasing acoustic frequencies. Thus, the pedestal may be at least partly due to the backscatter from microstructures on the animal. [Since the fine structure of the animal, (such as the legs) is much smaller than the acoustic wavelength, any scattering off the small parts would depend strongly on wavelength, and hence, frequency.]

We explore the effect that the pedestal has on the plots of theoretical beamwidth versus $k a$ and $L / \lambda$ by: 
1) distinguishing the average contributions to the echo due to the principle or smoothed body as $\bar{\sigma}_{\text {body }}$, and the microstructure as $\bar{\sigma}_{\mu}$,

2) assuming that the cross sections add incoherently.

The backscatter cross section for the animal is then the sum of the backscattering cross section from the "body" and from the microstructure:

$$
\bar{\sigma}_{\text {animal }}=\bar{\sigma}_{\text {body }}+\bar{\sigma}_{\mu} .
$$

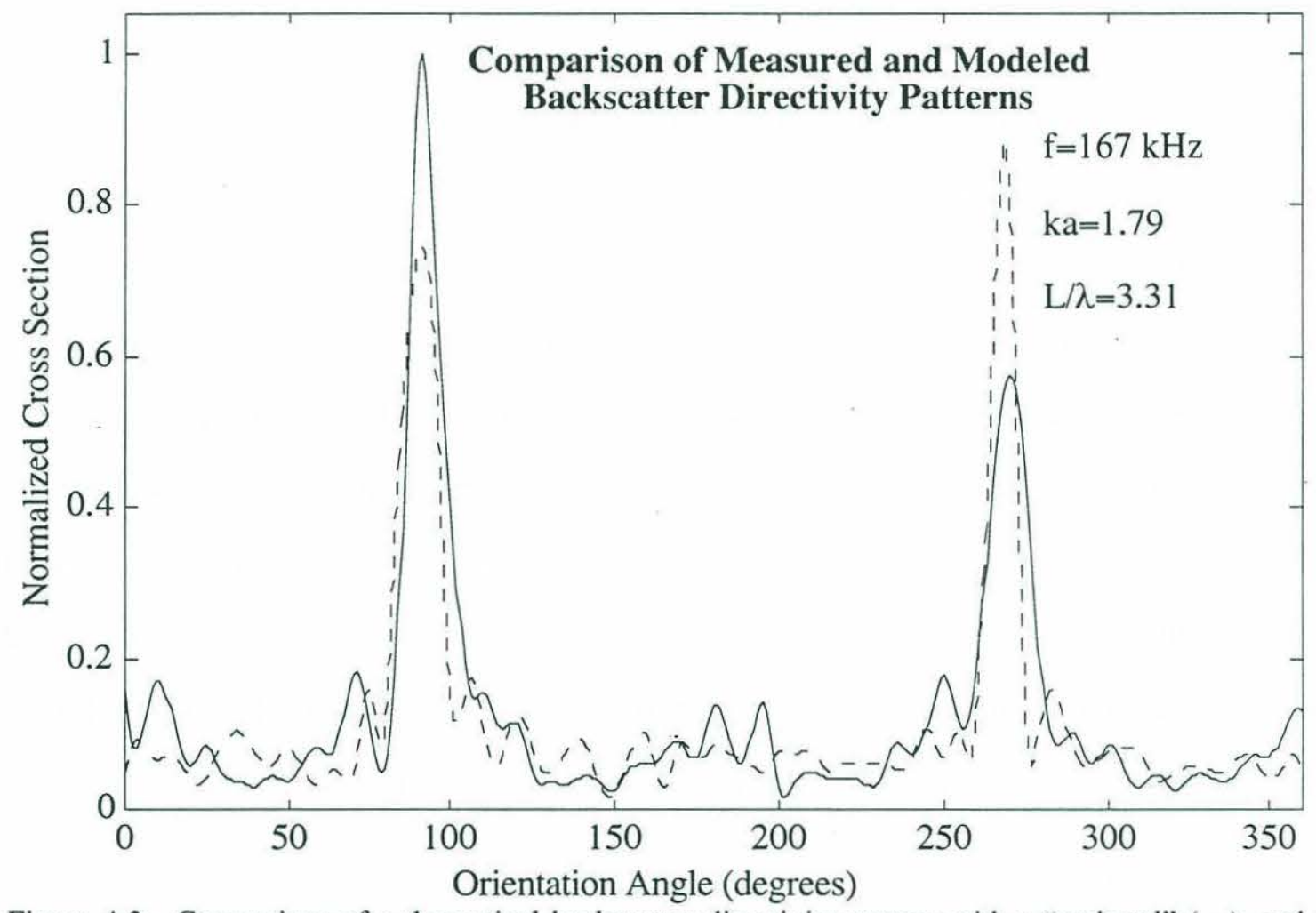

Figure 4-3: Comparison of a theoretical backscatter directivity pattern with a "pedestal" (-), and an experimentally determined backscatter directivity pattern $(--)$.

Figure 4-3 compares an actual backscatter directivity pattern from an animal with the backscatter directivity pattern from a model that includes a pedestal. For the theoretical backscatter directivity pattern, the wave form corresponding to the pedestal used was a zero-mean Gaussian random variable (random phase and amplitude) with a variance ranging from 0.00 to 0.20 , averaged over five pings. The variance was placed at 0.00 for an acoustic frequency of $50 \mathrm{kHz}$ and at 0.20 for an acoustic frequency of $500 \mathrm{kHz}$, and 
allowed to vary linearly between these two points. This is to reflect the observed increase in pedestal magnitude with respect to the main lobe height as acoustic frequency increased. (A linear change in variance was used over the frequency range due to the paucity of data - a frequency-to-the-fourth-power relationship between pedestal size and acoustic frequency may be more appropriate; this would cause only minute changes in the theoretical calculations of beamwidth.)

This improved model was used to generate the theoretical curves in Fig. 4-4 in the same fashion that the previous model was used to generate Fig. 4-1. Comparing Figs. 4-1 and 4-4, it is apparent that the correction for the pedestal slightly improved the comparison between the measured and modeled data.

Finally, the phenomenon of side lobe growth and merger into main lobes made the main lobe width difficult to define. The arbitrary definition of main lobe width used in this thesis added some artificiality to the study. As an alternative, the area under the curve of normalized backscatter directivity patterns was used as a more robust way to examine the data properties. This area is defined as the "normalized mean cross section". For an impenetrable plate, the normalized mean cross section goes to zero as $k a$ is increased. While the normalized mean cross section for the "ideal" prolate spheroid [Fig. 2-8, Eq. (2111)], and the experimental data (Figs. 3-11 and 3-12) tended level off (Fig. 4-5). 

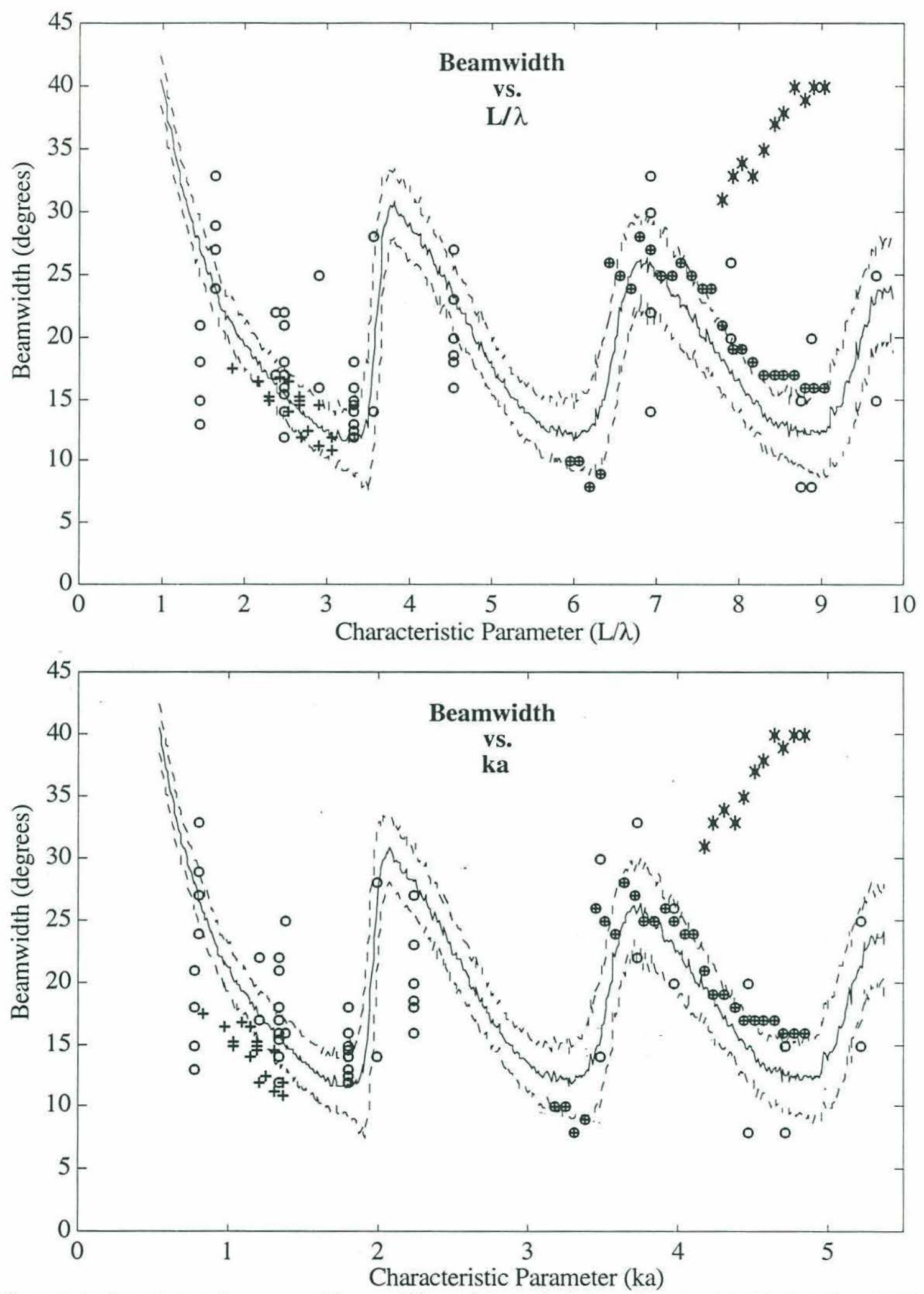

Figure 4-4: Experimentally measured beamwidths and theoretical data with a "pedestal" plotted against $k a$. and $L / \lambda$. ('o' denotes narrowband data; ' $*$ ' and ' $\approx$ ' denote chirp data, the ' $*$ ' data involves including a "rogue" side lobe in the estimate of beamwidth; and '+' denotes Greenlaw's data. The theoretical data has modeled animal motion, sampling limitations, and appendage motion.) 


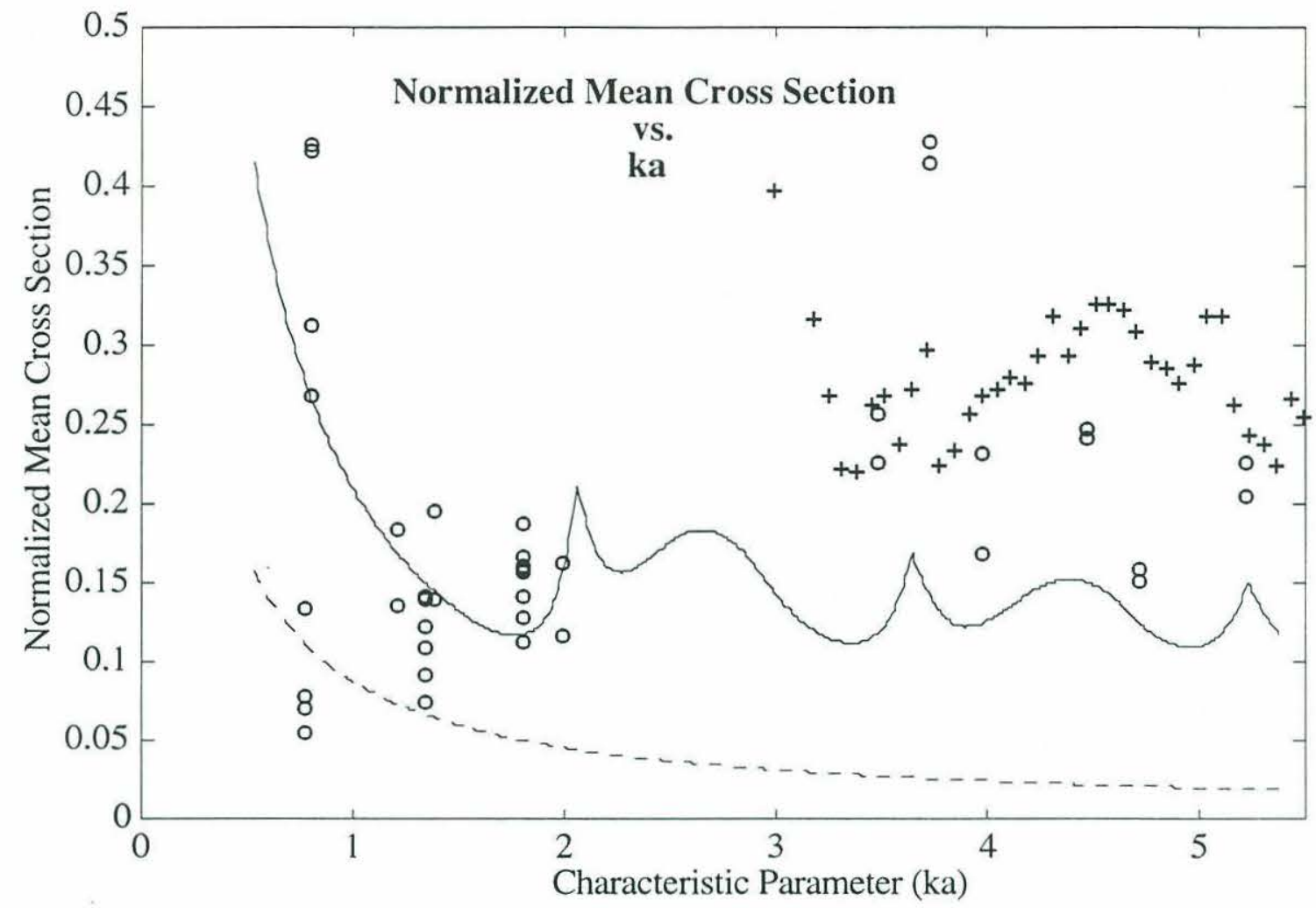

Figure 4-5: Comparison of the normalized mean cross section of the "ideal" non-moving prolate spheroid $(-)$, an impenetrable plate (- -), and experimental data ('o' denotes narrowband data and '+' denotes chirp data). 


\section{Chapter 5}

\section{Conclusions}

The data presented show the usefulness of the weakly scattering prolate spheroid for modeling the acoustic backscatter from an elongated zooplankton up to the values $k a \approx 4$ and $L / \lambda \approx 8$. The observed beamwidths generally fell within the maximum and minimum values predicted by the model. Above the values of $k a \approx 4$ and $L / \lambda \approx 8$ large features in the directivity pattern, presumably from the animal's microstructure, caused the prolate spheroid model to deviate from the data.

An interesting phenomenon observed (both theoretically and experimentally) was a set of step increases in beamwidth due to a substantial increase in level of the nearest side lobes in the vicinity of $k a=2(L / \lambda=3.5)$ and $k a=3.4(L / \lambda=6.4)$. These step increases in beamwidth as acoustic frequency is increased, are in sharp contrast to the trend predicted for impenetrable flat plates where a monotonic decrease in beamwidth with increasing acoustic frequency occurs [26]. As a general rule, the model based upon a flat plate underestimated the measured widths for zooplankton at all frequencies. This contrast is observed whether beamwidth (Fig. 2-8) or a more robust "first order" analysis involving the area under the normalized backscatter directivity patterns (Fig. 4-5) is measured.

The increase in the level of side lobes (the "pedestal") with increasing acoustic frequency was observed. This inspired an empirical modification to the otherwise oversimplified prolate spheroid model. In so doing, the resultant modified prolate spheroid model is able to predict beamwidths and average side lobe levels over a wide range of frequencies. 
The data sets collected represent only twenty-five measured backscatter directivity patterns over an acoustic frequency range of 72 to $525 \mathrm{kHz}$. More data sets are required over a larger frequency range and with more closely spaced intervals to further our understanding of the scattering phenomenon. In addition, sufficient beamwidths need to be measured to support a characteristic parameter that accounts for the animal size and shape with respect to the acoustic wavelength. (The parameters $k a$ and $L / \lambda$ were of limited value in applying the results to more general cases.)

Zooplankton are usually found in large populations whose orientation may or may not be chaotic. In order to study the populations acoustically, accurate scattering models need to be used. Critical to the development is the inclusion of the orientation dependence of the acoustic backscatter of elongated zooplankton. The results of this thesis represent a major step toward understanding the orientation dependence. 


\section{Bibliography}

[1] Barnes, Robert D. Invertebrate Zoology. 4th ed. Philadelphia: Holt, Rinehart and Winston, 1980.

[2] Chu, Dezhang, Timothy K. Stanton, and Peter H. Wiebe. "Frequency dependence of sound backscattering from live individual zooplankton," ICES J. mar. Sci. 49, (1992): 97-106.

[3] Stanton, Timothy K., Dezhang Chu, Peter H. Wiebe, and Clarence S. Clay. "Average echoes from randomly-oriented random-length finite cylinders: Zooplankton models," Journal of the Acoustical Society of America, in press (1993).

[4] Stanton, Timothy K., Clarence S. Clay, and Dezhang Chu. "Ray representation of sound scattering by weakly scattering deformed fluid cylinders: Simple physics and application to zooplankton," Journal of the Acoustical Society of America, in press (1993).

[5] Clay, Clarence S., and Herman Medwin. Acoustical Oceanography: Principles and Applications. New York: John Wiley and Sons; 1977.

[6] Stanton, Timothy K. "Sound scattering by zooplankton," Rapp. P.-v.Réun. Cons. int. Explor. Mer. 189, (1990): 353-362.

[7] Holliday, D. V. "Extracting bio-physical information from acoustic signatures of marine organisms," Oceanic Sound Scattering Prediction, N. R. Anderson, Ed. (1977): 619-624.

[8] Holliday, D. V., R. E. Pieper, and G. S. Kleppel "Determination of zooplankton size and distribution with multifrequency acoustic technology," Journal of Plankton Research, 32, (1989): 52-61.

[9] Pieper, R. E., D. V. Holliday, and G. S. Kleppel "Quantitative zooplankton distributions from multifrequency acoustics," Journal du Conseil International pour l'Exploration de la Mer, 49, (1990): 433-441.

[10] Anderson, Victor C. "Sound scattering from a fluid sphere," Journal of the Acoustical Society of America, 22, (1950): 426-431.

[11] Greenlaw, Charles F. "Backscattering spectra of preserved zooplankton," Journal of the Acoustical Society of America, 62, (1977): 44-52.

[12] Stanton, Timothy K. "Sound scattering by cylinders of finite length. I. Fluid cylinders," Journal of the Acoustical Society of America, 83, (1988): 55-63.

[13] Stanton, Timothy K. "Sound scattering by cylinders of finite length. II. Elastic cylinders," Journal of the Acoustical Society of America, 83, (1988): 64-67. 
[14] Stanton, Timothy K. "Sound scattering by cylinders of finite length. III. Deformed cylinders," Journal of the Acoustical Society of America, 86, (1989): 691-705.

[15] Greenlaw, Charles F. 1990. Private communication.

[16] Skudrzyk, Eugen. The Foundations of Acoustics. New York: Springer-Verlag, 1971.

[17] Morse, Philip M., and K. Uno Ingard. Theoretical Acoustics. Princeton, New Jersey: Princeton University Press, 1986.

[18] Davis, Harry F., and Arthur D. Snider. Introduction to Vector Analysis. 4th ed. Boston: Allyn and Bacon, 1979.

[19] Foote, Kenneth G. "Speed of sound in Euphausia superba," Journal of the Acoustical Society of America, 87, (1990): 1405-1408.

[20] Foote, Kenneth G., I. Everson, J. L. Watkins, and D. G. Bone "Target strengths of Antarctic krill (Euphausia superba) at 38 and $120 \mathrm{kHz}$, "Journal of the Acoustical Society of America, 87, (1990): 16-24.

[21] Ishimaru, Akira. Wave Propagation and Scattering in Random Media. San Diego: Academic Press, 1978.

[22] Morse, Philip M., and Herman Feshbach. Methods of Theoretical Physics. New York: McGraw-Hill, 1953.

[23] Hildebrand, Francis B. Advanced Calculus for Applications. 2nd ed. Englewood Cliffs, New Jersey: Prentice-Hall, 1976.

[24] Bateman, Harry. Tables of Integral Transforms, ed. A. Erdélyi. New York: McGraw-Hill, 1954.

[25] Abramowitz, Milton, Irene A. Stegun,.eds. Handbook of Mathematical Functions. New York: Dover Publications, 1972.

[26] Urick, Robert J. Principles of Underwater Sound. New York: McGraw-Hill, 1983.

[27] Pierce, Allan D. Acoustics: An Introduction to Its Physical Principles and Applications. New York: McGraw-Hill, 1981.

[28] Anderson, Victor C., and John C. Munson. "Directivity of spherical receiving arrays," Journal of the Acoustical Society of America, 35, (1963): 1162-1168.

[29] Queen, William C. "The directivity of sonar receiving arrays," Journal of the Acoustical Society of America, 47, (1970): 711-720.

[30] Burdic, William S. Underwater Acoustic System Analysis. Englewood Cliffs, New Jersey: Prentice-Hall, 1991.

[31] Doeblin, Ernest O. Measurement Systems: Application and Design. New York: McGraw-Hill, 1983. 
[32] Beckwith, Thomas G., N. Lewis Buck, and Roy D. Marangoni Mechanical Measurement . Reading, Massachusetts: Addison-Wesley, 1982.

[33] Stanton, Timothy K., and Clarence S. Clay. "Sonar echo statistics as a remote sensing tool: volume and seafloor," IEEE J. Ocean. Eng., OE-11, (1986): 79-96.

[34] Kennedy, John B., Adam M. Neville. Basic Statistical Methods for Engineers and Scientists. New York: Harper and Row, 1976.

[35] Oppenheim, Alan V., and Ronald W. Schafer. Discrete-Time Signal Processing. Englewood Cliffs, New Jersey: Prentice-Hall, 1989.

[36] Siebert, William M. Circuits, Signals, and Systems. New York: McGraw-Hill, 1986.

[37] Dahlquist, Germund, Åke Björck, and Ned Anderson. Numerical Methods. Englewood Cliffs, New Jersey: Prentice-Hall, 1974.

[38] Baker, Arthur de C., Brian P. Boden, and Edward Brinton. A Practical Guide to Euphausiids of the World London: Natural History Museum Publications, 1990.

[39] Coates, Rodney F. W. Underwater Acoustic Systems. New York: John Wiley and Sons, 1989. 